\title{
On the justification of the quasistationary approximation in the problem of motion of a viscous capillary drop
}

\author{
V. A. SOLONNIKOV \\ V.A. Steklov Institute of Mathematics, St Petersburg Department, \\ Fontanka, 27, 191011 St Petersburg, Russia
}

[Received 25 May 1998 and in revised form 8 January 1999]

\begin{abstract}
We prove that the free boundary problem governing the motion of an isolated liquid mass in the case of a small Reynolds number $\varepsilon$ has a unique solution in a certain time interval $\left(0, T_{0}\right)$ independent of $\varepsilon$ and we show that the difference of the solution and of the quasistationary approximation to the solution has order $O(\varepsilon)$ for $t \in\left(t_{0}, T_{0}\right)$ with arbitrary positive $t_{0}$.
\end{abstract}

\section{Introduction}

The problem considered in the present paper consists of the determination of a bounded domain $\Omega_{t}, t>0$, which is given at the initial moment of time $t=0$, of the vector field of velocity $\mathbf{v}(x, t)=\left(v_{1}, v_{2}, v_{3}\right)$ and of a scalar pressure $p(x, t)$ satisfying in $\Omega_{t}$ the Navier-Stokes equations (in a dimensionless form)

$$
\varepsilon\left(\mathbf{v}_{t}+(\mathbf{v} \cdot \nabla) \mathbf{v}\right)-\nabla^{2} \mathbf{v}+\nabla p=0, \quad \nabla \cdot \mathbf{v}=0 \quad\left(x \in \Omega_{t}, \quad t>0\right),
$$

initial conditions

$$
\mathbf{v}(x, 0)=\mathbf{v}_{0}(x) \quad\left(x \in \Omega_{0}\right),
$$

and conditions at the free (unknown) boundary $\Gamma_{t}=\partial \Omega_{t}$

$$
\left.\mathbf{v} \cdot \mathbf{n}\right|_{\Gamma_{t}}=V_{n}, \quad T(\mathbf{v}, p) \mathbf{n}-\left.H \mathbf{n}\right|_{\Gamma_{t}}=0 .
$$

Here $V_{n}$ is the velocity of the motion of the free surface $\Gamma_{t}$ in the direction of the exterior (with respect to $\Omega_{t}$ ) normal $\mathbf{n}, H$ is the doubled mean curvature of $\Gamma_{t}$, negative for convex surfaces, $T(\mathbf{v}, p)=-p I+S(\mathbf{v})$ is the stress tensor, $S(\mathbf{v})=\left(\frac{\partial v_{i}}{\partial x_{j}}+\frac{\partial v_{j}}{\partial x_{i}}\right)_{i, j=1,2,3}$ is the doubled rate-of-strain tensor, $I$ is a unit matrix, and $\varepsilon$ is a small positive parameter.

The first kinematic boundary condition (1.3) means that at every point of the surface $\Gamma_{t}$ the velocity of evolution of this surface in the direction $\mathbf{n}$ coincides with the normal component of the velocity of the liquid. The second (dynamic) condition says that the tangential stresses at the free boundary vanish, and the normal stress is equilibreated with a capillary force. According to (1.1), there are no external forces acting on the drop $\Omega_{t}$.

For a fixed positive $\varepsilon$, problem (1.1)-(1.3) has been studied in [12-15]. It has been shown that the solution exists in a certain finite time interval, however, if $\Omega_{0}$ is close to a ball and $\mathbf{v}_{0}(x)$ is small, then it is defined for all positive values of time, and a limiting regime as $t \rightarrow \infty$, depending on initial data, is rotation of the liquid as a rigid body about a certain axis moving with a constant speed. It has been also proved that for $t \rightarrow \infty, \Omega_{t}$ tends to a circle in the case of two spacial variables and to a certain equilibrium figure in the three-dimensional case.

(c) Oxford University Press 1999 
In [5] an important special case of a two-dimensional problem (1.1)-(1.3) is studied, namely, the problem of motion of a ring filled with a viscous capillary liquid. In this case it turned out possible to make a complete analysis of the behaviour of the solution for $t>0$, without any smallness assumptions on the data.

In a recent paper [4] by M. Günther and G. Prokert the same problem (1.1)-(1.3) is considered in a quasistationary approximation. This approximate model consists in the elimination of the terms $\mathbf{v}_{t}+(\mathbf{v} \cdot \nabla) \mathbf{v}$ in (1.1) by setting $\varepsilon=0$ and of initial conditions (1.2) for $\mathbf{v}(x, t)$. Thus, the problem reduces to the solution of a linear elliptic (for $\epsilon=0$ ) system (1.1) in a time-dependent domain $\Omega_{t}$ whose evolution is determined completely by conditions (1.3). It has been proved in [4] that the solution of this problem is determined in a certain finite time interval, or for all $t>0$, if $\Omega_{0}$ is close to a ball. Another proof of the local solvability of the same problem in the Hölder spaces was given in [17]. The discussion of the range of applicability of quasistationary approximation is contained in [9].

In the present paper we prove that the problem (1.1)-(1.3) is solvable in a certain finite time interval independent of $\varepsilon \in\left(0, \varepsilon_{0}\right), \varepsilon_{0} \ll 1$. This is done by comparing $(\mathbf{v}, p)$ with a quasistationary approximation $(\mathbf{w}, r)$ and by making a better analysis of the corresponding linear problem than has been done in $[7,8,16]$. In comparison with these papers, our arguments here are much simpler. For the differences $\mathbf{v}-\mathbf{w}, \quad p-r$ written as functions of the Lagrangean coordinates we obtain representation formulas (1.18) and estimates (1.14), (4.20), (1.20) which show that these differences have order $O(\varepsilon)$ for $t>t_{0}, \forall t_{0}>0$. This provides justification of the quasistationary approximation.

The relation between the Eulerian coordinates $x=\left(x_{1}, x_{2}, x_{3}\right) \in \Omega_{t}$ and the Lagrangean coordinates $\xi=\left(\xi_{1}, \xi_{2}, \xi_{3}\right) \in \Omega_{0} \equiv \Omega$ is given by the formula

$$
\mathbf{x}=\boldsymbol{\xi}+\int_{0}^{t} \mathbf{u}(\xi, \tau) \mathrm{d} \tau \equiv \mathbf{X}_{u}(\xi, t), \quad \xi \in \Omega,
$$

where $\mathbf{x}$ and $\xi$ are radii-vectors corresponding to the points $x$ and $\xi$, and $\mathbf{u}(\xi, t)$ is the velocity vector field written as a function of the Lagrangean coordinates. This formula determines a mapping $x=X_{u}(\xi, t)$ of $\Omega$ onto $\Omega_{t}$ and of $\Gamma_{0} \equiv \Gamma$ onto $\Gamma_{t}$ (sometimes, in order to avoid confusion, we denote $\Gamma_{t}$ by $\Gamma_{u}(t)$ and the normal $\mathbf{n}$ to $\Gamma_{t}$ by $\mathbf{n}_{u}$ ). By virtue of $\nabla \cdot \mathbf{v}=0$, this mapping is invertible, and the determinant of its Jacobi matrix equals 1. It tranforms (1.1)-(1.3) into

$$
\begin{gathered}
\varepsilon \mathbf{u}_{t}-\nabla_{u}^{2} \mathbf{u}+\nabla_{u} q=0, \quad \nabla_{u} \cdot \mathbf{u}=0, \quad \xi \in \Omega, \quad t>0, \\
\mathbf{u}(\xi, 0)=\mathbf{v}_{0}(\xi), \\
T_{u}(\mathbf{u}, q) \mathbf{n}-\left.H \mathbf{n}\right|_{\Gamma}=0
\end{gathered}
$$

(condition $\mathbf{v} \cdot \mathbf{n}=V_{n}$ is equivalent to $X_{u}(\Omega)=\Omega_{t}$ ). Here

$$
\nabla_{u}=\mathcal{A} \nabla=\left(\sum_{j=1}^{3} A_{i j} \frac{\partial}{\partial \xi_{j}}\right)_{i=1,2,3},
$$

$A_{i j}$ is a cofactor of the element $a_{i j}(\xi, t)=\delta_{i j}+\int_{0}^{t} \frac{\partial u_{i}}{\partial \xi_{j}} \mathrm{~d} \tau$ of the Jacobi matrix of the transformation $X_{u}, q(\xi, t)=p(X(\xi, t), t), \quad T_{u}(\mathbf{u}, q)=-q I+S_{u}(\mathbf{u})$, and

$$
S_{u}(\mathbf{u})=\left(\sum_{k=1}^{3}\left(A_{i k} \frac{\partial u_{j}}{\partial \xi_{k}}+A_{j k} \frac{\partial u_{i}}{\partial \xi_{k}}\right)\right)_{i, j=1,2,3} .
$$


It is convenient to modify (1.5) by introducing some additional terms into the Navier-Stokes equations and by using the well known formula $H \mathbf{n}=\Delta_{u}(t) \mathbf{x}=\Delta_{u}(t) \mathbf{X}_{u}(\xi, t)$ where $\Delta_{u}(t)$ is the Laplace-Beltrami operator on $\Gamma_{t}$. Instead of (1.5), we consider the problem

$$
\begin{gathered}
\varepsilon \mathbf{u}_{t}+\ell_{u}(\mathbf{u})-\nabla_{u}^{2} \mathbf{u}+\nabla_{u} q=\sum_{k=1}^{6} \mu_{k} \boldsymbol{\varphi}_{k}\left(X_{u}(\xi, t)\right), \\
\nabla_{u} \cdot \mathbf{u}=0, \quad(\xi \in \Omega, \quad t>0), \\
\mathbf{u}(\xi, 0)=\mathbf{v}_{0}(\xi), \\
T_{u}(\mathbf{u}, q) \mathbf{n}-\left.\Delta_{u}(t) \mathbf{X}_{u}\right|_{\Gamma}=0
\end{gathered}
$$

where $\varphi_{k}(x), k=1, \ldots, 6$, are linearly independent vector fields in the space of vectors $\varphi(x)=$ $\mathbf{a}+\mathbf{b} \times \mathbf{x}$ of rigid displacement, for instance, $\varphi_{1}=(1,0,0)=\mathbf{e}_{1}, \varphi_{2}=(0,1,0)=\mathbf{e}_{2}, \varphi_{3}=$ $(0,0,1)=\mathbf{e}_{3}, \varphi_{4}=\left(0,-x_{3}, x_{2}\right), \varphi_{5}=\left(x_{3}, 0,-x_{1}\right), \varphi_{6}=\left(-x_{2}, x_{1}, 0\right)$,

$$
\begin{gathered}
\ell_{u}(\mathbf{u})=\sum_{k=1}^{6}\left(\int_{\Omega} \mathbf{u}\left(\xi^{\prime}, t\right) \cdot \varphi_{k}\left(X_{u}\left(\xi^{\prime}, t\right)\right) \mathrm{d} \xi^{\prime}\right) \varphi_{k}\left(X_{u}(\xi, t)\right), \\
\mu_{k}=\int_{\Omega} \mathbf{v}_{0}(x) \cdot \varphi_{k}(x) \mathrm{d} x .
\end{gathered}
$$

Multiplying (1.61) by $\varphi_{m}\left(X_{u}(\xi, t)\right)$ and integrating over $\Omega$, we obtain (see details in [12])

$$
\varepsilon \frac{\mathrm{d} \lambda_{m}}{\mathrm{~d} t}+\sum_{k=1}^{6} \lambda_{k}(t) \int_{\Omega_{t}} \boldsymbol{\varphi}_{k} \cdot \boldsymbol{\varphi}_{m} \mathrm{~d} x=0
$$

where

$$
\lambda_{k}(t)=\int_{\Omega_{t}} \mathbf{v}(y, t) \cdot \varphi_{k}(y) \mathrm{d} y-\mu_{k}=\int_{\Omega} \mathbf{u}(\xi, t) \cdot \boldsymbol{\varphi}_{k}\left(X_{u}(\xi, t)\right) \mathrm{d} \xi-\mu_{k},
$$

and, as a consequence,

$$
\begin{gathered}
\frac{\varepsilon}{2} \frac{\mathrm{d}}{\mathrm{d} t} \sum_{m=1}^{6} \lambda_{m}^{2}(t)+c \sum_{m=1}^{6} \lambda_{m}^{2}(t) \\
\leqslant \frac{\varepsilon}{2} \frac{\mathrm{d}}{\mathrm{d} t} \sum_{m=1}^{6} \lambda_{m}^{2}(t)+\sum_{k, m=1}^{6} \lambda_{k}(t) \lambda_{m}(t) \int_{\Omega_{t}} \boldsymbol{\varphi}_{k} \cdot \boldsymbol{\varphi}_{m} \mathrm{~d} x=0 .
\end{gathered}
$$

Since $\lambda_{m}(0)=0$, we conclude that $\lambda_{m}(t)=0$, i.e.

$$
\int_{\Omega} \mathbf{u}(\xi, t) \cdot \varphi_{m}\left(X_{u}(\xi, t)\right) \mathrm{d} \xi=\mu_{m}
$$

for all $t>0$, so (1.6) is equivalent to (1.5).

We prove the solvability of this problem by comparing $(\mathbf{u}, q)$ with the solution $(\mathbf{w}, r)$ of the same problem in a quasistationary approximation $[4,17]$. In the Lagrangean coordinates, the latter problem can be written as follows:

$$
-\nabla_{w}^{2} \mathbf{w}+\nabla_{w} r+\ell_{w}(\mathbf{w})=\sum_{k=1}^{6} \mu_{k} \varphi\left(X_{w}(\xi, t)\right)
$$




$$
\begin{gathered}
\nabla_{w} \cdot \mathbf{w}=0, \quad \xi \in \Omega, t>0, \\
T_{w}(\mathbf{w}, q) \mathbf{n}_{w}-\left.\Delta_{w}(t) \mathbf{X}_{w}(\xi, t)\right|_{\Gamma}=0 .
\end{gathered}
$$

Here $\mathbf{X}_{w}(\xi, t)=\boldsymbol{\xi}+\int_{0}^{t} \mathbf{w}(\xi, t) \mathrm{d} t, \quad \Delta_{w}(t)$ is the Laplace-Beltrami operator on $\Gamma_{w}(t)=X_{w}(\Gamma)$, $\mathbf{n}_{w}$ is a unit exterior normal to $\Gamma_{w}(t), \mu_{k}$ are the same numbers as in (1.7) and

$$
\ell_{w}(\mathbf{v})=\sum_{k=1}^{6}\left(\int_{\Omega} \mathbf{v}\left(\xi^{\prime}, t\right) \cdot \boldsymbol{\varphi}_{k}\left(X_{w}\left(\xi^{\prime}, t\right)\right) \mathrm{d} \xi^{\prime}\right) \boldsymbol{\varphi}_{k}\left(X_{w}(\xi, t)\right)
$$

The solvability of problem (1.8) on a certain finite time interval in the Hölder spaces of functions was established in [17] where the following theorem was proved.

THEOREM 1.1 Let $\Gamma \in C^{3+l}$ where $l$ is an arbitrary fixed positive non-integral number. There exists such $T_{0}>0$ that problem (1.8) is uniquely solvable on the time interval $\left(0, T_{0}\right)$. The solution satisfies the conditions

$$
\int_{\Omega} \mathbf{w}(\xi, t) \cdot \boldsymbol{\varphi}_{k}\left(X_{w}(\xi, t)\right) \mathrm{d} \xi=\mu_{k}, \quad k=1, \ldots, 6,
$$

and the inequality

$$
\sup _{t<T_{0}}|\mathbf{w}(\cdot, t)|_{C^{2+l}(\Omega)}+\sup _{t<T_{0}}|r(\cdot, t)|_{C^{1+l}(\Omega)} \leqslant c\left(\left|H_{0}\right|_{C^{l+1}(\Gamma)}+\sum_{k=1}^{6}\left|\mu_{k}\right|\right)
$$

where $H_{0}$ is the doubled mean curvature of $\Gamma$.

Here $C^{l}(\Omega)$ means a standard Hölder space of functions (or vector fields) $u(x), x \in \Omega$, with the norm

$$
\begin{aligned}
|u|_{C^{l}(\Omega)} & =\sum_{|j|<l} \sup _{\Omega}\left|D^{j} u(x)\right|+[u]_{\Omega}^{(l)}, \\
{[u]_{\Omega}^{(l)} } & =\sum_{|j|=[l]} \sup _{x, y \in \Omega}|x-y|^{-l+[l]}\left|D^{j} u(x)-D^{j} u(y)\right|,
\end{aligned}
$$

and $C^{l}(\Gamma)$ is defined, as usual, with the help of partition of unity on $\Gamma$ and of local maps. The norm in the space $C^{l, l / 2}(\Omega \times(0, T))$ may be defined by the formula

$$
|u|_{C^{l, l / 2}(\Omega \times(0, T))}=\sup _{t \in(0, T)}|u(\cdot, t)|_{C^{l}(\Omega)}+\sup _{\Omega}[u(x, \cdot)]_{(0, T)}^{(l / 2)}
$$

but most often another equivalent norm in this space is used; it contains maxima moduli and Hölder constants of the mixed derivatives $D_{t}^{k} D_{x}^{j} u(x, t), 2 k+|j|<l$. Finally, by $\tilde{C}\left(0, T ; C^{l}(\Omega)\right)$ we mean the space of functions (or vector fields) continuous with respect to $(x, t) \in \Omega \times(0, T)$ and having a finite norm

$$
\sup _{t \in(0, T)}|u(\cdot, t)|_{\left.C^{l}(\Omega)\right)}
$$

(unfortunately, in [17] a misleading notation $C\left(0, T ; C^{l}(\Omega)\right)$ for this space was used). 
We also consider two linear evolution problems:

$$
\begin{gathered}
\varepsilon \mathbf{V}_{t}-\nabla_{w}^{2} \mathbf{V}+\ell_{w}(\mathbf{V})+\nabla_{w} P=0, \\
\nabla_{w} \cdot \mathbf{V}=0, \quad \xi \in \Omega, \quad t<T_{0}, \\
\left.\mathbf{V}\right|_{t=0}=\mathbf{v}_{0}(\xi)-\mathbf{u}_{0}(\xi) \equiv \mathbf{V}_{0}(\xi), \\
\left.T_{w}(\mathbf{V}, P) \mathbf{n}_{w}\right|_{\Gamma}=0
\end{gathered}
$$

and

$$
\begin{gathered}
\varepsilon \boldsymbol{\eta}_{t}-\nabla_{w}^{2} \boldsymbol{\eta}+\ell_{w}(\boldsymbol{\eta})+\nabla_{w} \pi=\mathbf{f}(\xi, t), \\
\nabla_{w} \cdot \boldsymbol{\eta}=g(\xi, t), \quad \xi \in \Omega, \quad t<T_{0}, \\
\left.\boldsymbol{\eta}\right|_{t=0}=0, \\
\left.\Pi_{w} S_{w}(\boldsymbol{\eta}) \mathbf{n}_{w}\right|_{\Gamma}=\mathbf{b}, \\
\mathbf{n}_{w} \cdot T_{w}(\boldsymbol{\eta}, \pi) \mathbf{n}_{w}-\left.\mathbf{n}_{w} \cdot \Delta_{w}(t) \int_{0}^{t} \boldsymbol{\eta} \mathrm{d} \tau\right|_{\Gamma}=b+\int_{0}^{t} B(\xi, \tau) \mathrm{d} \tau
\end{gathered}
$$

where $\Pi_{w}$ is a projector onto the tangent plane to $\Gamma_{w}(t)$ at the point $X_{w}(\xi, t)$, i.e.

$$
\Pi_{w} \mathbf{f}=\mathbf{f}-\mathbf{n}_{w}\left(\mathbf{n}_{w} \cdot \mathbf{f}\right) .
$$

By $\Pi_{0}$ we mean a projector onto the tangent plane to $\Gamma$ at the point $\xi$, i.e.

$$
\Pi_{0} \mathbf{f}=\mathbf{f}-\mathbf{n}_{0}\left(\mathbf{n}_{0} \cdot \mathbf{f}\right),
$$

$\mathbf{n}_{0}$ being the exterior normal to $\Gamma$.

We prove the following theorems.

THEOREM 1.2 If $\Gamma \in C^{3+\alpha}, \mathbf{v}_{0} \in C^{2+\alpha}(\Omega) \quad(\alpha \in(0,1))$ and $\nabla \cdot \mathbf{V}_{0}(\xi)=0,\left.\Pi_{0} S\left(\mathbf{V}_{0}\right) \mathbf{n}_{0}\right|_{\Gamma}=$ 0 , then problem (1.11) has a unique solution in the interval $\left(0, T_{0}\right)$, and the solution satisfies the inequalities

$$
\begin{gathered}
\varepsilon \sup _{\tau<t}\left|\mathbf{V}_{\tau}(\cdot, \tau)\right|_{C^{\alpha}(\Omega)}+\sup _{\tau<t}|\mathbf{V}(\cdot, \tau)|_{C^{2+\alpha}(\Omega)}+\sup _{\tau<t}|P(\cdot, \tau)|_{C^{l+1}(\Omega)} \leqslant c\left|\mathbf{V}_{0}\right|_{C^{2+\alpha}(\Omega)}, \\
\|\mathbf{V}(\cdot, t)\|_{L_{2}(\Omega)} \leqslant c \|\left.\mathbf{V}_{0}\right|_{L_{2}(\Omega)} \exp \left(-b t \varepsilon^{-1}\right), \quad b>0, \quad t \in\left(0, T_{0}\right), \\
\int_{0}^{T_{0}}|\mathbf{V}|_{C^{2+\alpha}(\Omega)} \mathrm{d} t \leqslant c \varepsilon\left|\mathbf{V}_{0}\right|_{C^{2+\alpha}(\Omega)} .
\end{gathered}
$$

If $\Gamma \in C^{4+\alpha}$, then also

$$
\int_{0}^{T_{0}}|\mathbf{V}|_{C^{3+\alpha}(\Gamma)} \mathrm{d} t \leqslant c \varepsilon\left|\mathbf{V}_{0}\right|_{C^{2+\alpha}(\Omega)} .
$$

The constants in (1.13)-(1.16) are independent of $\varepsilon$. 
THEOREM 1.3 Assume that $\Gamma \in C^{3+\alpha}, \mathbf{f} \in \tilde{C}\left(0, T_{0} ; C^{\alpha}(\Omega)\right), g \in \tilde{C}\left(0, T_{0} ; C^{1+\alpha}(\Omega)\right)$, $g(\xi, t)=\nabla \cdot \mathbf{h}(\xi, t)+h_{0}(\xi, t), \mathbf{h}_{t} \in \tilde{C}\left(0, T_{0} ; C^{\alpha}(\Omega)\right), h_{0 t} \in C\left(0, T_{0} ; C(\Omega)\right)$, $\mathbf{b} \in C^{1+\alpha,(1+\alpha) / 2}\left(\Gamma \times\left(0, T_{0}\right)\right), b \in \tilde{C}\left(0, T_{0} ; C^{1+\alpha}(\Gamma)\right), B \in \tilde{C}\left(0, T_{0} ; C^{\alpha}(\Gamma)\right)$, and $\mathbf{b}, \quad g$ satisfy the conditions

$$
\mathbf{b} \cdot \mathbf{n}_{0}=0, \quad \mathbf{b}(\xi, 0)=0, \quad g(\xi, 0)=0 .
$$

Then problem (1.12) has a unique solution $\eta \in \tilde{C}\left(0, T_{0} ; C^{2+\alpha}(\Omega)\right), \pi \in \tilde{C}\left(0, T_{0} ; C^{1+\alpha}(\Omega)\right)$ such that $\eta_{t} \in \tilde{C}\left(0, T_{0} ; C^{\alpha}(\Omega)\right)$, and

$$
\begin{aligned}
\varepsilon \sup _{\tau<t}\left|\boldsymbol{\eta}_{\tau}(\cdot, \tau)\right|_{C^{\alpha}(\Omega)} & +\sup _{\tau<t}|\boldsymbol{\eta}(\cdot, \tau)|_{C^{2+\alpha}(\Omega)}+\sup _{\tau<t}|\pi(\cdot, \tau)|_{C^{1+\alpha}(\Omega)} \leqslant \\
\leqslant & c\left(\sup _{\tau<t}|\mathbf{f}(\cdot, \tau)|_{C^{\alpha}(\Omega)}+\sup _{\tau<t}|g(\cdot, \tau)|_{C^{1+\alpha}(\Omega)}+\varepsilon \sup _{\tau<t}\left|\mathbf{h}_{\tau}(\cdot, \tau)\right|_{C^{\alpha}(\Omega)}\right. \\
& +\varepsilon \sup _{\Omega \times(0, t)}\left|h_{0 \tau}(x, \tau)\right|+\sup _{\tau<t}|\mathbf{b}(\cdot, \tau)|_{C^{1+\alpha}(\Gamma)}+\sup _{\tau<t}|b(\cdot, \tau)|_{C^{1+\alpha}(\Gamma)} \\
& \left.+\varepsilon^{(1+\alpha) / 2} \sup _{\Gamma}[\mathbf{b}(\xi, \cdot)]_{(0, t)}^{(1+\alpha) / 2)}+\sup _{\tau<t}|B(\cdot, \tau)|_{C^{\alpha}(\Gamma)}\right)
\end{aligned}
$$

for arbitrary $t \in\left(0, T_{0}\right)$.

To prove the solvability of non-linear problem (1.6), we introduce new unknown functions

$$
\boldsymbol{\eta}=\mathbf{u}-\mathbf{w}-\mathbf{V}, \quad \pi=q-r-P .
$$

It is easy to see that (1.6) is equivalent to the problem

$$
\begin{gathered}
\varepsilon \boldsymbol{\eta}_{t}-\nabla_{w}^{2} \boldsymbol{\eta}+\ell_{w}(\boldsymbol{\eta})+\nabla_{w} \pi=-\varepsilon \mathbf{w}_{t}+\mathcal{L}_{1}(\boldsymbol{\eta}, \pi), \\
\nabla_{w} \cdot \boldsymbol{\eta}=\mathcal{L}_{2}(\boldsymbol{\eta}), \quad \xi \in \Omega, \quad t<T_{0}, \\
\left.\boldsymbol{\eta}\right|_{t=0}=0, \\
\left.\Pi_{w} S_{w}(\boldsymbol{\eta}) \mathbf{n}_{w}\right|_{\Gamma}=\mathcal{L}_{3}(\boldsymbol{\eta}), \\
\mathbf{n}_{w} \cdot T_{w}(\boldsymbol{\eta}, \pi) \mathbf{n}_{w}-\left.\mathbf{n}_{w} \cdot \Delta_{w}(t)\left(\mathbf{X}_{u}-\mathbf{X}_{w}\right)\right|_{\Gamma}=\mathcal{L}(\boldsymbol{\eta}, \pi)
\end{gathered}
$$

where

$$
\begin{gathered}
\mathcal{L}_{1}(\boldsymbol{\eta}, \pi)=\left(\nabla_{u}^{2}-\nabla_{w}^{2}\right) \mathbf{u}-\left(\nabla_{u}-\nabla_{w}\right) q \\
+\ell_{w}(\mathbf{u})-\ell_{u}(\mathbf{u})+\sum_{k=1}^{6} \mu_{k}\left(\boldsymbol{\varphi}_{k}\left(X_{u}(\xi, t)\right)-\boldsymbol{\varphi}_{k}\left(X_{w}(\xi, t)\right),\right. \\
\mathcal{L}_{2}(\boldsymbol{\eta})=\left(\nabla_{w}-\nabla_{u}\right) \cdot \mathbf{u}, \\
\mathcal{L}_{3}(\boldsymbol{\eta})=\Pi_{w}\left(\Pi_{w} S_{w}(\mathbf{u}) \mathbf{n}_{w}-\Pi_{u} S_{u}(\mathbf{u}) \mathbf{n}_{u}\right), \\
\mathcal{L}(\boldsymbol{\eta}, \pi)=\mathbf{n}_{w} \cdot\left(T_{w}(\mathbf{u}, q) \mathbf{n}_{w}-T_{u}(\mathbf{u}, q) \mathbf{n}_{u}\right)+\mathbf{n}_{w} \cdot\left(\Delta_{u}(t)-\Delta_{w}(t)\right) \mathbf{X}_{u}(\xi, t)
\end{gathered}
$$

and $\mathbf{u}=\mathbf{w}+\mathbf{V}+\boldsymbol{\eta}, \quad q=r+P+\pi$.

Applying Theorem 1.2 and using estimates of the coefficients of the operators $\nabla_{u}-\nabla_{w}$ and $\Delta_{u}(t)-\Delta_{w}(t)$, we prove the following main result of the paper. 
THEOREM 1.4 If $\Gamma \in C^{4+\alpha}$, then problem (1.19) has a unique solution $\eta, \pi$ in a certain time interval $t \in\left(0, T_{1}\right), T_{1} \leqslant T_{0}$, and the solution satisfies the inequality

$$
\varepsilon \sup _{\tau<t}\left|\boldsymbol{\eta}_{\tau}(\cdot, \tau)\right|_{C^{\alpha}(\Omega)}+\sup _{\tau<t}|\boldsymbol{\eta}(\cdot, \tau)|_{C^{2+\alpha}(\Omega)}+\sup _{\tau<t}|\pi(\cdot, \tau)|_{C^{1+\alpha}(\Omega)} \leqslant c \varepsilon
$$

with arbitrary $t \in\left(0, T_{1}\right)$ and with the constant $c$ independent of $\varepsilon$.

This theorem guarantees the existence of solution $\mathbf{u}=\boldsymbol{\eta}+\mathbf{w}+\mathbf{V}, q=\pi+r+P$ of problem (1.6) for $t \in\left(0, T_{1}\right)$, moreover, the difference $\mathbf{u}-\mathbf{w}$ is represented as the sum of a boundary layer type function $\mathbf{V}$ (at $t=0$ ) and of the vector field $\boldsymbol{\eta}$ for which uniform estimate (1.20) is obtained. Hence, for $t>t_{0}>0$ this difference does not exceed $c\left(t_{0}\right) \varepsilon$.

Finally, we prove that the solution of problem (1.6) can be extended into the whole interval $t \in\left(0, T_{0}\right)$.

The paper is organized as follows. In Sections 2 and 3 we study a linear problem

$$
\begin{gathered}
\varepsilon \mathbf{v}_{t}-\nabla^{2} \mathbf{v}+\ell(\mathbf{v})+\nabla p=\mathbf{f}, \\
\nabla \cdot \mathbf{v}=g=\nabla \cdot \mathbf{h}, \quad x \in \Omega, \quad t \in(0, T), \\
\left.\mathbf{v}\right|_{t=0}=0, \\
\left.\Pi_{0} S(\mathbf{v}) \mathbf{n}_{0}\right|_{\Gamma}=\mathbf{b}, \\
\mathbf{n}_{0} \cdot T(\mathbf{v}, p) \mathbf{n}_{0}-\left.\mathbf{n}_{0} \cdot \Delta_{0} \int_{0}^{t} \mathbf{v} \mathrm{d} \tau\right|_{\Gamma}=b+\int_{0}^{t} B \mathrm{~d} \tau
\end{gathered}
$$

where $\ell(\mathbf{v})=\sum_{k=1}^{6}\left(\int_{\Omega} \mathbf{v}\left(\xi^{\prime}, t\right) \cdot \boldsymbol{\varphi}_{k}\left(\xi^{\prime}, t\right) \mathrm{d} \xi^{\prime}\right) \boldsymbol{\varphi}_{k}(\xi, t), \Delta_{0}$ is the Laplace-Beltrami operator on $\Gamma$, and the corresponding model problem in the half-space $\mathbb{R}_{+}^{3}=\left\{x_{3}>0\right\}$. In the case of a fixed $\varepsilon>0$, this problem was considered earlier in $[7,8,16]$, in particular, in anisotropic Hölder spaces $C^{2+\alpha, 1+\alpha / 2}(\Omega \times(0, T))$, and one of the most difficult technical points was the estimate of the Hölder constants of the time derivative of $\mathbf{v}$ with respect to $t$. For our purposes here a more modest estimate of the norm

$$
Y_{t}(\mathbf{v}, p)=\varepsilon \sup _{\tau<t}\left|\mathbf{v}_{\tau}(\cdot, \tau)\right|_{C^{\alpha}(\Omega)}+\sup _{\tau<t}|\mathbf{v}(\cdot, \tau)|_{C^{2+\alpha}(\Omega)}+\sup _{\tau<t}|p(\cdot, \tau)|_{C^{1+\alpha}(\Omega)}
$$

is sufficient, and it is proved by simpler arguments. For instance, we do not use the theory of Fourier multipliers in the Hölder spaces which is replaced here by Proposition 2.2 on the pointwise estimates of the kernels (2.27) made in the spirit of [1]. The final results of Sections 2 and 3 are contained in the following theorem.

TheORem 1.5 Let $\Gamma \in C^{2+\alpha}, \mathbf{f} \in \tilde{C}\left(0, T ; C^{\alpha}(\Omega)\right), g \in \tilde{C}\left(0, T ; C^{\alpha+1}(\Omega)\right), \mathbf{h}_{t} \in$ $\tilde{C}\left(0, T ; C^{\alpha}(\Omega)\right), h_{0 t} \in C(\Omega \times(0, T)), \mathbf{b} \in C^{1+\alpha,(1+\alpha) / 2}(\Gamma \times(0, T)), b \in \tilde{C}\left(0, T ; C^{1+\alpha}(\Gamma)\right)$, $B \in \tilde{C}\left(0, T ; C^{\alpha}(\Gamma)\right)$, and let $\mathbf{b}, \quad g$ satisfy the compatibility conditions

$$
\mathbf{b} \cdot \mathbf{n}_{0}=0, \quad \mathbf{b}(x, 0)=0, \quad g(x, 0)=0 .
$$


Then problem (1.21)-(1.22) has a unique solution $\mathbf{v} \in \tilde{C}\left(0, T ; C^{2+\alpha}(\Omega)\right), p \in \tilde{C}\left(0, T ; C^{1+\alpha}(\Omega)\right)$ with $\mathbf{v}_{t} \in \tilde{C}\left(0, T ; C^{\alpha}(\Omega)\right)$, and the solution satisfies the inequality

$$
\begin{aligned}
Y_{t}(\mathbf{v}, p) \leqslant & c\left(\sup _{\tau<t}|\mathbf{f}(\cdot, \tau)|_{C^{\alpha}(\Omega)}+\sup _{\tau<t}|g(\cdot, \tau)|_{C^{1+\alpha}(\Omega)}\right. \\
& +\sup _{\tau<t}\left|\mathbf{h}_{\tau}(\cdot, \tau)\right|_{C^{\alpha}(\Omega)}+\sup _{\tau<t} \sup _{\Omega}\left|h_{0 \tau}(\xi, \tau)\right|+\sup _{\tau<t}|\mathbf{b}(\cdot, \tau)|_{C^{1+\alpha}(\Gamma)} \\
& \left.+\varepsilon^{(1+\alpha) / 2} \sup _{\Gamma}[\mathbf{b}(\xi, \cdot)]_{(0, t)}^{((1+\alpha) / 2)}+\sup _{\tau<t}|b(\cdot, \tau)|_{C^{1+\alpha}(\Gamma)}+\sup _{\tau<t}|B(\cdot, \tau)|_{C^{\alpha}(\Gamma)}\right) .
\end{aligned}
$$

We also consider the initial-boundary value problem for (1.21) with initial and boundary conditions

$$
\begin{gathered}
\left.\mathbf{v}\right|_{t=0}=\mathbf{v}_{0}(\xi), \\
\left.T(\mathbf{v}, p) \mathbf{n}_{0}\right|_{\Gamma}=\mathbf{a} .
\end{gathered}
$$

Theorem 1.6 Let $\Gamma \in C^{2+\alpha}, \mathbf{f} \in \tilde{C}\left(0, T ; C^{\alpha}(\Omega)\right), g \in \tilde{C}\left(0, T ; C^{\alpha+1}(\Omega)\right), \mathbf{h}_{t} \in$ $\tilde{C}\left(0, T ; C^{\alpha}(\Omega)\right), h_{0 t} \in C(\Omega \times(0, T)), \mathbf{v}_{0} \in C^{2+\alpha}(\Omega), \Pi_{0} \mathbf{a} \in C^{1+\alpha,(1+\alpha) / 2}(\Gamma \times(0, T))$, $\mathbf{a} \cdot \mathbf{n}_{0} \in \tilde{C}\left(0, T ; C^{1+\alpha}(\Gamma)\right)$, and let the compatibility conditions

$$
\nabla \cdot \mathbf{v}_{0}(x)=g(x, 0),\left.\quad \Pi_{0} \mathbf{v}_{0}(x)\right|_{\Gamma}=\Pi_{0} \mathbf{a}(x, 0)
$$

be satisfied. Then problem (1.21)-(1.24) has a unique solution $\mathbf{v} \in \tilde{C}\left(0, T ; C^{2+\alpha}(\Omega)\right), p \in$ $\tilde{C}\left(0, T ; C^{1+\alpha}(\Omega)\right)$ with $\mathbf{v}_{t} \in \tilde{C}\left(0, T ; C^{\alpha}(\Omega)\right)$, and the solution satisfies the inequality

$$
\begin{aligned}
Y_{t}(\mathbf{v}, p) \leqslant & c\left(\sup _{\tau<t}|\mathbf{f}(\cdot, \tau)|_{C^{\alpha}(\Omega)}+\sup _{\tau<t}|g(\cdot, \tau)|_{C^{1+\alpha}(\Omega)}\right. \\
& +\sup _{\tau<t}\left|\mathbf{h}_{\tau}(\cdot, \tau)\right|_{C^{\alpha}(\Omega)}+\sup _{\tau<t} \sup _{\Omega}\left|h_{0 \tau}(\xi, \tau)\right|+\left|\mathbf{v}_{0}\right|_{C^{2+\alpha}(\Omega)} \\
& \left.+\sup _{\tau<t}|\mathbf{a}(\cdot, \tau)|_{C^{1+\alpha}(\Gamma)}+\varepsilon^{(1+\alpha) / 2} \sup _{\Gamma}\left[\Pi_{0} \mathbf{a}(\xi, \cdot)\right]_{(0, t)}^{((1+\alpha) / 2)}\right) .
\end{aligned}
$$

For a fixed $\varepsilon>0$, problem (1.21)-(1.24) is considered in [10,11] and estimate (1.25) is proved in [18]. A uniform (with respect to $\varepsilon>0$ ) estimate (1.25) is obtained in the same way as the inequality (1.23), and we omit the details.

In Section 4 we consider the problem (1.8), estimate the time derivative $\mathbf{w}_{t}(\xi, t)$ and prove theorems 1.2 and 1.3. Finally, in Section 5 we prove Theorem 1.4.

\section{Model problem in the half-space}

\subsection{Construction of the solution}

In this section we consider the model initial-boundary value problem in the half-space $\mathbb{R}_{+}^{3}\left(x_{3}>0\right)$ :

$$
\begin{aligned}
& \varepsilon \mathbf{v}_{t}-\nabla^{2} \mathbf{v}+\nabla p=0, \quad \nabla \cdot \mathbf{v}=0 \quad\left(x \in \mathbb{R}_{+}^{3}, t>0\right), \\
& \left.\mathbf{v}\right|_{t=0}=0,
\end{aligned}
$$




$$
\begin{aligned}
& \frac{\partial v_{j}}{\partial x_{3}}+\left.\frac{\partial v_{3}}{\partial x_{j}}\right|_{x_{3}=0}=b_{j}\left(x^{\prime}, t\right), \quad j=1,2, \\
& -p+2 \frac{\partial v_{3}}{\partial x_{3}}+\left.\Delta^{\prime} \int_{0}^{t} v_{3}(x, t) \mathrm{d} \tau\right|_{x_{3}=0}=b_{3}^{\prime}\left(x^{\prime}, t\right) \quad\left(x^{\prime}=\left(x_{1}, x_{2}\right) \in \mathbb{R}^{2}, \quad t>0\right),
\end{aligned}
$$

where $\Delta^{\prime}=\frac{\partial^{2}}{\partial x_{1}^{2}}+\frac{\partial^{2}}{\partial x_{2}^{2}}$. We assume that $b_{1}, b_{2}, b_{3}^{\prime}$ are smooth functions decaying at infinity sufficiently rapidly. Making the Fourier transform with respect to $x^{\prime}$ and the Laplace transform with respect to $t$ defined by the formula

$$
F L h \equiv \widetilde{h}\left(\xi, x_{3}, t\right)=\int_{0}^{\infty} \int_{\mathbb{R}^{2}} e^{-i x^{\prime} \xi} e^{-s t} h\left(x^{\prime}, x_{3}, t\right) \mathrm{d} x^{\prime} \mathrm{d} t
$$

we reduce (2.1)-(2.2) to the boundary value problem for the system of ordinary differential equations

$$
\begin{aligned}
& -\frac{\mathrm{d}^{2} \widetilde{v}_{j}}{\mathrm{~d} x_{3}^{2}}+r_{\varepsilon}^{2} \widetilde{v}_{j}+i \xi_{j} \widetilde{p}=0, \quad j=1,2, \\
& -\frac{\mathrm{d}^{2} \widetilde{v}_{3}}{\mathrm{~d} x_{3}^{2}}+r_{\varepsilon}^{2} \widetilde{v}_{3}+\frac{\mathrm{d} \widetilde{p}}{\mathrm{~d} x_{3}}=0, \\
& i \xi_{1} \widetilde{v}_{1}+i \xi_{2} \widetilde{v}_{2}+\frac{\mathrm{d} \widetilde{v}_{3}}{\mathrm{~d} x_{3}}=0, \quad x_{3}>0, \\
& \widetilde{v}_{k}\left(\xi, x_{3}, s\right) \rightarrow 0, \quad \widetilde{p}\left(\xi, x_{3}, s\right) \rightarrow 0 \quad\left(x_{3} \rightarrow+\infty\right), \\
& \quad \frac{\mathrm{d} \widetilde{v}_{j}}{\mathrm{~d} x_{3}}+\left.i \xi_{j} \widetilde{v}_{3}\right|_{x_{3}=0}=\widetilde{b}_{j}(\xi, s), \quad j=1,2, \\
& \quad-\widetilde{p}+2 \frac{\mathrm{d} \widetilde{v}_{3}}{\mathrm{~d} x_{3}}-\left.\frac{|\xi|^{2}}{s} \widetilde{v}_{3}\right|_{x_{3}=0}=\widetilde{b}_{3}^{\prime}(\xi, s),
\end{aligned}
$$

where $r_{\varepsilon}=\sqrt{\varepsilon s+|\xi|^{2}}, \operatorname{Re} r_{\varepsilon} \geqslant 0$ for $\operatorname{Re} s \geqslant 0$.

We also consider the initial-boundary value problem for the Stokes equations (2.1) with boundary conditions (2.2) and (2.5) replaced by

$$
\begin{aligned}
& \frac{\partial v_{j}}{\partial x_{3}}+\left.\frac{\partial v_{3}}{\partial x_{j}}\right|_{x_{3}=0}=a_{j}\left(x^{\prime}, t\right), \quad j=1,2 \\
& -p+\left.2 \frac{\partial v_{3}}{\partial x_{3}}\right|_{x_{3}=0}=a_{3}\left(x^{\prime}, t\right) \\
& \frac{\mathrm{d} \widetilde{v}_{j}}{\mathrm{~d} x_{3}}+\left.i \xi_{j} \widetilde{v}_{3}\right|_{x_{3}=0}=\widetilde{a}_{j}(\xi, s), \quad j=1,2 \\
& -\tilde{p}+\left.2 \frac{\mathrm{d} \widetilde{v}_{3}}{\mathrm{~d} x_{3}}\right|_{x_{3}=0}=\widetilde{a}_{3}(\xi, s)
\end{aligned}
$$


As shown in [10], problem (2.4)-(2.7) can be solved explicitly in the form

$$
\begin{aligned}
\tilde{v}_{i}\left(\xi, x_{3}, s\right)= & \frac{\left(1-\delta_{3 i}\right) \tilde{a}_{i}}{r_{\varepsilon}} e^{-r_{\varepsilon} x_{3}}+\sum_{j=1}^{3} \frac{R_{i j}\left(r_{\varepsilon}, \xi\right)}{r_{\varepsilon} Q\left(r_{\varepsilon},|\xi|\right)} \tilde{a}_{j} e^{-r_{\varepsilon} x_{3}} \\
& +\sum_{j=1}^{3} \frac{S_{i j}\left(r_{\varepsilon}, \xi\right)}{Q\left(r_{\varepsilon},|\xi|\right)} \widetilde{a}_{j} \frac{e^{-r_{\varepsilon} x_{3}}-e^{-|\xi| x_{3}}}{r_{\varepsilon}-|\xi|}, \\
\widetilde{p}\left(\xi, x_{3}, s\right)= & -\tilde{a}_{3} e^{-|\xi| x_{3}}+\sum_{j=1}^{3} P_{j}\left(r_{\varepsilon}, \xi\right) \tilde{a}_{j} e^{-|\xi| x_{3}}
\end{aligned}
$$

where

$$
\begin{aligned}
& P_{j}\left(r_{\varepsilon}, \xi\right)=2 i \xi_{j} \frac{r_{\varepsilon}\left(r_{\varepsilon}+|\xi|\right)}{Q\left(r_{\varepsilon},|\xi|\right)}, \quad j=1,2, \\
& P_{3}\left(r_{\varepsilon}, s\right)=2|\xi|^{2} \frac{r_{\varepsilon}-|\xi|}{Q\left(r_{\varepsilon},|\xi|\right)}, \\
& Q\left(r_{\varepsilon},|\xi|\right)=r_{\varepsilon}^{3}+|\xi| r_{\varepsilon}^{2}+3 r_{\varepsilon}|\xi|^{2}-|\xi|^{3}=\left(r_{\varepsilon}+|\xi|\right) M(\varepsilon s,|\xi|) \\
& M(\varepsilon s,|\xi|)=\varepsilon s+4|\xi|^{2} \frac{r_{\varepsilon}}{r_{\varepsilon}+|\xi|}
\end{aligned}
$$

and $R_{i j}, S_{i j}$ are elements of the matrices

$$
\begin{aligned}
& \mathcal{R}=\left(\begin{array}{ccc}
\xi_{1}^{2}\left(3 r_{\varepsilon}-|\xi|\right) & \xi_{1} \xi_{2}\left(3 r_{\varepsilon}-|\xi|\right) & i \xi_{1} r_{\varepsilon}\left(r_{\varepsilon}-|\xi|\right) \\
\xi_{1} \xi_{2}\left(3 r_{\varepsilon}-|\xi|\right) & \xi_{2}^{2}\left(3 r_{\varepsilon}-|\xi|\right) & i \xi_{2} r_{\varepsilon}\left(r_{\varepsilon}-|\xi|\right) \\
-i \xi_{1} r_{\varepsilon}\left(r_{\varepsilon}-|\xi|\right) & -i \xi_{2} r_{\varepsilon}\left(r_{\varepsilon}-|\xi|\right) & -|\xi| r_{\varepsilon}\left(r_{\varepsilon}+|\xi|\right)
\end{array}\right), \\
& \mathcal{S}=\left(\begin{array}{ccc}
-2 r_{\varepsilon} \xi_{1}^{2} & -2 r_{\varepsilon} \xi_{1} \xi_{2} & -i \xi_{1}\left(r_{\varepsilon}^{2}+|\xi|^{2}\right) \\
-2 r_{\varepsilon} \xi_{1} \xi_{2} & -2 r_{\varepsilon} \xi_{2}^{2} & -i \xi_{2}\left(r_{\varepsilon}^{2}+|\xi|^{2}\right) \\
-2 i \xi_{1}|\xi| r_{\varepsilon} & -2 i \xi_{2}|\xi| r_{\varepsilon} & |\xi|\left(r_{\varepsilon}^{2}+|\xi|^{2}\right)
\end{array}\right) .
\end{aligned}
$$

Hence,

$$
\begin{aligned}
-\tilde{p}+2 \frac{\mathrm{d} \widetilde{v}_{3}}{\mathrm{~d} x_{3}}-\left.\frac{|\xi|^{2}}{s} \widetilde{v}_{3}\right|_{x_{3}=0} & =\widetilde{a}_{3}-\frac{|\xi|^{2}}{s} \sum_{j=1}^{3} \frac{R_{3 j}\left(r_{\varepsilon},|\xi|\right)}{r_{\varepsilon} Q\left(r_{\varepsilon},|\xi|\right)} \widetilde{a}_{j} \\
& =\tilde{a}_{3}\left(1+\frac{|\xi|^{3}}{s M(\varepsilon s,|\xi|)}\right)+\frac{|\xi|^{2}}{s M(\varepsilon s,|\xi|)} \frac{r_{\varepsilon}-|\xi|}{r_{\varepsilon}+|\xi|} \sum_{j=1}^{2} i \xi_{j} \tilde{a}_{j} .
\end{aligned}
$$

It follows that the solution of problem (2.4)-(2.5) may be considered as a solution of problem (2.4)-(2.7) with $\widetilde{a}_{1}=\widetilde{b}_{1}, \widetilde{a}_{2}=\widetilde{b}_{2}$ and

$$
\begin{aligned}
\widetilde{a}_{3} & =\frac{s M(\varepsilon s,|\xi|)}{P_{\varepsilon}(s,|\xi|)} \widetilde{b}_{3}^{\prime}-\frac{|\xi|^{2}}{P_{\varepsilon}(s,|\xi|)} \frac{r_{\varepsilon}-|\xi|}{r_{\varepsilon}+|\xi|} \sum_{j=1}^{2} i \xi_{j} \widetilde{b}_{j} \\
& =\widetilde{b}_{3}^{\prime}\left(1-\frac{|\xi|^{3}}{P_{\varepsilon}(s,|\xi|)}\right)-\frac{|\xi|^{2}}{P_{\varepsilon}(s,|\xi|)} \frac{r_{\varepsilon}-|\xi|}{r_{\varepsilon}+|\xi|} \sum_{j=1}^{2} i \xi_{j} \tilde{b}_{j}
\end{aligned}
$$




$$
P_{\varepsilon}(s,|\xi|)=s M(\varepsilon s,|\xi|)+|\xi|^{3} .
$$

Making the inverse Fourier-Laplace transformation

$$
F^{-1} L^{-1} \widetilde{h} \equiv h\left(x^{\prime}, x_{3}, t\right)=\frac{1}{(2 \pi)^{2} 2 \pi i} \int_{\mathbb{R}^{2}} e^{i x \cdot \xi} \mathrm{d} \xi \int_{\operatorname{Re} s=a \geqslant 0} e^{s t} \widetilde{h}\left(\xi, x_{3}, s\right) \mathrm{d} s
$$

we find that (2.11) is equivalent to

$$
a_{3}\left(x^{\prime} . t\right)=\sum_{j=1}^{2}\left(V * \frac{\partial b_{j}}{\partial x_{j}}\right)+b_{3}^{\prime}+\sum_{j=1}^{2}\left(V_{j} * \frac{\partial b_{3}^{\prime}}{\partial x_{j}}\right)
$$

where

$$
\widetilde{V}=-\frac{|\xi|^{2}}{P_{\varepsilon}} \frac{r_{\varepsilon}-|\xi|}{r_{\varepsilon}+|\xi|}, \quad \widetilde{V}_{j}=\frac{i \xi_{j}|\xi|}{P_{\varepsilon}}
$$

and the convolution is taken with respect to $x^{\prime}$ and $t$.

The initial-boundary value problem for the non-homogeneous Stokes equations

$$
\begin{aligned}
& \varepsilon \mathbf{v}_{t}-\nabla^{2} \mathbf{v}+\nabla p=\mathbf{f}(x, t), \quad \nabla \cdot \mathbf{v}=g(x, t) \quad\left(x \in \mathbb{R}_{+}^{3}, \quad t \in(0, T)\right), \\
& \left.\mathbf{v}\right|_{t=0}=\mathbf{v}_{0}(x), \\
& \frac{\partial v_{j}}{\partial x_{3}}+\left.\frac{\partial v_{3}}{\partial x_{j}}\right|_{x_{3}=0}=b_{j}\left(x^{\prime}, t\right), \quad j=1,2, \\
& -p+2 \frac{\partial v_{3}}{\partial x_{3}}+\left.\Delta^{\prime} \int_{0}^{t} v_{3}(x, t) \mathrm{d} \tau\right|_{x_{3}=0}=b_{3}^{\prime}\left(x^{\prime}, t\right) \quad\left(x^{\prime}=\left(x_{1}, x_{2}\right) \in \mathbb{R}^{2}, \quad t \in(0, T)\right),
\end{aligned}
$$

can be reduced to problem (2.1)-(2.2) by construction of auxiliary functions

$$
\begin{aligned}
& \mathbf{v}^{(1)}(x, t)=\int_{0}^{t} \mathrm{~d} \tau \int_{\mathbb{R}^{3}} \Gamma_{\varepsilon}(x-y, t-\tau) \mathbf{f}^{*}(y, \tau) \mathrm{d} y+\varepsilon \int_{\mathbb{R}^{3}} \Gamma_{\varepsilon}(x-y, t) \mathbf{v}_{0}^{*}(y) \mathrm{d} y, \\
& \mathbf{v}^{(2)}(x, t)=\nabla \int_{\mathbb{R}_{+}^{3}}\left(E(x-y)-E\left(x-y^{*}\right)\right)\left(g(y, t)-\nabla \cdot \mathbf{v}^{(1)}(y, t)\right) \mathrm{d} y, \\
& p^{(2)}=g(x, t)-\nabla \cdot \mathbf{v}^{(1)}(x, t)-\varepsilon \int_{\mathbb{R}_{+}^{3}}\left(E(x-y)-E\left(x-y^{*}\right)\right)\left(g_{t}-\nabla \cdot \mathbf{v}_{t}^{(1)}\right) \mathrm{d} y .
\end{aligned}
$$

Here $\mathbf{f}^{*}$ and $\mathbf{v}_{0}^{*}$ are extensions of the functions $\mathbf{f}$ and $\mathbf{v}_{0}$, respectively, into the domain $\left\{x_{3}<0\right\}$, $\Gamma_{\varepsilon}(x, t)=\varepsilon^{-1}\left(4 \pi t \varepsilon^{-1}\right)^{-3 / 2} \exp \left(-\varepsilon|x|^{2} / 4 t\right)$ and $E(x)=-\frac{1}{4 \pi|x|}$ are fundamental solutions of the heat and Laplace equations, respectively, and $y^{*}=\left(y_{1}, y_{2},-y_{3}\right)$. It can be easily seen that

$$
\begin{gathered}
\varepsilon \mathbf{v}_{t}^{(1)}-\nabla^{2} \mathbf{v}^{(1)}=\mathbf{f}^{*}(x, t), \quad \mathbf{v}^{(1)}(x, 0)=\mathbf{v}_{0}^{*}(x), \\
\nabla \cdot \mathbf{v}^{(2)}=g-\nabla \cdot \mathbf{v}^{(1)}, \\
\varepsilon \mathbf{v}_{t}^{(2)}-\nabla^{2} \mathbf{v}^{(2)}+\nabla p^{(2)}(x, t)=0,\left.\quad v_{1}^{(2)}\right|_{x_{3}=0}=\left.v_{2}^{(2)}\right|_{x_{3}=0}=0,
\end{gathered}
$$


and, if the compatibility condition $g(x, 0)=\nabla \cdot \mathbf{v}_{0}(x)=\nabla \cdot \mathbf{v}^{(1)}(x, 0)$ is satisfied, then $\left.\mathbf{v}^{(2)}\right|_{t=0}=0$. Hence, for $\mathbf{v}^{(3)}=\mathbf{v}-\mathbf{v}^{(1)}-\mathbf{v}^{(2)}, \quad p^{(3)}=p-p^{(2)}$ we obtain the problem of the type (2.1)-(2.2):

$$
\begin{gathered}
\varepsilon \mathbf{v}_{t}^{(3)}-\nabla^{2} \mathbf{v}^{(3)}+\nabla p^{(3)}=0, \quad \nabla \cdot \mathbf{v}^{(3)}=0 \quad\left(x \in \mathbb{R}^{3}, \quad t \in(0, T)\right), \\
\frac{\partial v_{j}^{(3)}}{\partial x_{3}}+\left.\frac{\partial v_{3}^{(3)}}{\partial x_{j}}\right|_{x_{3}=0}=b_{j}\left(x^{\prime}, t\right)-\left.\left(\frac{\partial v_{j}^{(1)}}{\partial x_{3}}+\frac{\partial v_{3}^{(1)}}{\partial x_{j}}\right)\right|_{x_{3}=0}-\left.\left(\frac{\partial v_{j}^{(1)}}{\partial x_{3}}+\frac{\partial v_{3}^{(1)}}{\partial x_{j}}\right)\right|_{x_{3}=0}, \quad j=1,2, \\
-p^{(3)}+2 \frac{\partial v_{3}^{(3)}}{\partial x_{3}}+\left.\Delta^{\prime} \int_{0}^{t} v_{3}^{(3)}(x, \tau) \mathrm{d} \tau\right|_{x_{3}=0}= \\
=b_{3}^{\prime}\left(x^{\prime}, t\right)+\left.\left(p^{(2)}-2 \frac{\partial v_{3}^{(1)}}{\partial x_{3}}-2 \frac{\partial v_{3}^{(2)}}{\partial x_{3}}\right)\right|_{x_{3}=0}-\left.\Delta^{\prime} \int_{0}^{t} v_{3}^{(1)}(x, \tau) \mathrm{d} \tau\right|_{x_{3}=0} .
\end{gathered}
$$

If $\mathbf{f} g$ have compact supports, then $\mathbf{v}^{(1)}$ decays at infinity exponentially and $\mathbf{v}^{(2)}, p^{(2)}$ decay like power functions, at least, like $\left|x^{\prime}\right|^{-1}$.

\subsection{Auxiliary propositions}

We start with the proof of some auxiliary inequalities which are necessary for the estimate of convolution integrals in (2.14). We consider at first the function (2.12). We observe that the functions

$$
r(s, \xi)=\sqrt{s+|\xi|^{2}}, \quad \frac{r(s, \xi)}{r(s, \xi)+|\xi|}, \quad M(s,|\xi|)=s+4|\xi|^{2} \frac{r(s, \xi)}{r(s, \xi)+|\xi|}
$$

satisfy the inequalities

$$
\begin{gathered}
c_{1}\left(|s|+|\xi|^{2}\right)^{1 / 2} \leqslant \operatorname{Re} r(s, \xi) \leqslant|r(s, \xi)| \leqslant c_{2}\left(|s|+|\xi|^{2}\right)^{1 / 2}, \\
c_{3} \leqslant \operatorname{Re} \frac{r(s, \xi)}{r(s, \xi)+|\xi|} \leqslant c_{4}, \\
c_{5}\left(|s|+|\xi|^{2}\right) \leqslant|M(s, \xi)| \leqslant c_{6}\left(|s|+|\xi|^{2}\right)
\end{gathered}
$$

for arbitrary $\xi \in \mathbb{R}^{2}$ and $s \in \mathbb{C}$ such that

$$
\operatorname{Re} s+\kappa|\operatorname{Im} s|>-\delta|\xi|^{2}
$$

where $\delta$ and $\kappa$ are small positive numbers. Moreover, these inequalities (maybe, with other constants) hold true if $\xi_{j}$ is replaced with $\zeta_{j}=\xi_{j}+i \eta_{j}, \xi_{j}, \eta_{j} \in \mathbb{R}, j=1,2$, and

$$
|\eta| \leqslant \delta_{1}|\xi|
$$

where $\delta_{1}>0$ is a small positive number. With the help of (2.18)-(2.20) we can evaluate the function

$$
P^{(\tau)}(s,\langle\zeta\rangle)=s M(s,\langle\zeta\rangle)+\tau\langle\zeta\rangle^{3}
$$

where $\tau>0,\langle\zeta\rangle=\left(\zeta_{1}^{2}+\zeta_{2}^{2}\right)^{1 / 2}, \zeta_{j}=\xi_{j}+i \eta_{j}$. 
Proposition 2.1 Assume that

$$
\operatorname{Re} s+\kappa|\operatorname{Im} s| \geqslant a \gg \tau^{2}
$$

(see inequality (2.24)) and that condition (2.21) is satisfied. Then

$$
\begin{aligned}
c_{7}\left(|s|\left(|s|+|\xi|^{2}\right)+\tau|\xi|^{3}\right) & \leqslant\left|P^{(\tau)}(s,\langle\zeta\rangle)\right| \\
& \leqslant c_{8}\left(|s|\left(|s|+|\xi|^{2}\right)+\tau|\xi|^{3}\right) .
\end{aligned}
$$

Moreover, if $\delta_{2} / \tau$ and $\delta_{2} / \sqrt{a}$ are small enough, then

$$
\begin{aligned}
c_{9}\left(|s|\left(|s|+|\xi|^{2}\right)+\tau|\xi|^{3}\right) & \leqslant\left|P^{(\tau)}\left(s-\delta_{2}\langle\zeta\rangle,\langle\zeta\rangle\right)\right| \\
& \leqslant c_{10}\left(|s|\left(|s|+|\xi|^{2}\right)+\tau|\xi|^{3}\right)
\end{aligned}
$$

The constants $c_{7}-c_{10}$ are independent of $\tau$.

Proof. We start with the estimate of $P^{(\tau)}(s,|\xi|), \xi \in \mathbb{R}^{2}$, from below (the estimate from above is evident). We consider the sum

$$
\operatorname{Re} \frac{P^{(\tau)}(s,|\xi|)}{M(s,|\xi|)}+\kappa_{1}\left|\operatorname{Im} \frac{P^{(\tau)}(s,|\xi|)}{M(s,|\xi|)}\right|=\operatorname{Re}(s+T \bar{M})+\kappa_{1}|\operatorname{Im}(s+T \bar{M})|
$$

where $T=\tau|\xi|^{3} /|M|^{2}, \kappa_{1}>\kappa$, and $\kappa_{1}$ is small. We have

$$
T \leqslant \frac{\tau \sqrt{|s|}|\xi|^{3}}{\min \sqrt{|s|}|M|^{2}} \leqslant \frac{\left(1+\kappa^{2}\right)^{1 / 4}}{c_{5}^{2} \sqrt{a}} \frac{\tau \sqrt{|s|}|\xi|^{3}}{\left(|s|+|\xi|^{2}\right)^{2}} \leqslant \frac{\left(1+\kappa^{2}\right)^{1 / 4}}{c_{5}^{2}} \frac{\tau}{\sqrt{a}} \leqslant \frac{1}{2},
$$

if

$$
\frac{\tau}{\sqrt{a}} \leqslant \frac{c_{5}^{2}}{2\left(1+\kappa^{2}\right)^{1 / 4}}
$$

and, under this condition,

$$
\begin{aligned}
& \operatorname{Re} \frac{P^{(\tau)}(s,|\xi|)}{M(s,|\xi|)}+\kappa_{1}\left|\operatorname{Im} \frac{P^{(\tau)}(s,|\xi|)}{M(s,|\xi|)}\right| \geqslant \\
& \geqslant(1+T) \operatorname{Re} s+\kappa_{1}(1-T)|\operatorname{Im} s|+4|\xi|^{2} T\left(\operatorname{Re} \frac{r}{r+|\xi|}-\kappa_{1}\left|\operatorname{Im} \frac{r}{r+|\xi|}\right|\right) \\
& \geqslant \frac{1}{2}\left(\operatorname{Re} s+\kappa_{1}|\operatorname{Im} s|\right)+4|\xi|^{2} T\left(c_{3}-\kappa_{1} c_{4}\right) \\
& \geqslant c\left(|s|+4|\xi|^{2} T\right) \geqslant \frac{2}{3} c\left(|s|+T\left(|s|+4|\xi|^{2}\right)\right)
\end{aligned}
$$


provided that $\kappa_{1} \leqslant c_{3} / 2 c_{4}$. Hence,

$$
\operatorname{Re} \frac{P^{(\tau)}(s,|\xi|)}{M(s,|\xi|)}+\kappa_{1}\left|\operatorname{Im} \frac{P^{(\tau)}(s,|\xi|)}{M(s,|\xi|)}\right| \geqslant c\left(|s|+\frac{\tau|\xi|^{3}}{M \mid}\right)
$$

which implies

$$
\left|\frac{P^{(\tau)}(s,|\xi|)}{M(s,|\xi|)}\right| \geqslant \frac{c}{\sqrt{1+\kappa_{1}^{2}}}\left(|s|+\frac{\tau|\xi|^{3}}{|M|}\right)
$$

and gives a necessary estimate

$$
\left|P^{(\tau)}(s,|\xi|)\right| \geqslant c\left(|s|\left(|s|+|\xi|^{2}\right)+\tau|\xi|^{3}\right) .
$$

Inequality (2.22) follows from (2.25) and from

$$
\left|P^{(\tau)}(s,\langle\zeta\rangle)-P^{(\tau)}(s,|\xi|)\right| \leqslant c|\eta|\left(|s||\xi|+\tau|\xi|^{2}\right) \leqslant c \delta_{1}\left(|s||\xi|^{2}+\tau|\xi|^{3}\right),
$$

if $\delta_{1}$ is small.

Finally, to prove (2.23), we evaluate the difference

$$
\begin{aligned}
& P^{(\tau)}\left(s-\delta_{2}\langle\zeta\rangle,\langle\zeta\rangle\right)-P^{(\tau)}(s,\langle\zeta\rangle)=-\delta_{2}\langle\zeta\rangle \int_{0}^{1} \frac{\partial}{\partial s} P^{(\tau)}\left(s-\delta_{2} \lambda\langle\zeta\rangle,\langle\zeta\rangle\right) \mathrm{d} \lambda \\
& =-\delta_{2}\langle\zeta\rangle \int_{0}^{1}\left[M\left(s-\delta_{2} \lambda\langle\zeta\rangle,\langle\zeta\rangle\right)+\left(s-\delta_{2} \lambda\langle\zeta\rangle\right) \frac{\partial M\left(s-\delta_{2} \lambda\langle\zeta\rangle,\langle\zeta\rangle\right)}{\partial s}\right] \mathrm{d} \lambda .
\end{aligned}
$$

Since

$$
\operatorname{Re}\left(s-\delta_{2} \lambda\langle\zeta\rangle\right)+\kappa\left|\operatorname{Im}\left(s-\delta_{2} \lambda\langle\zeta\rangle\right)\right| \geqslant a-c \delta_{2}|\xi| \geqslant-\delta|\xi|^{2}+a-\frac{c^{2} \delta_{2}^{2}}{4 \delta} \geqslant-\delta|\xi|^{2},
$$

if $\delta_{2} / \sqrt{a} \leqslant 2 \sqrt{\delta} / c$, the functions $r\left(s-\delta_{2} \lambda\langle\zeta\rangle,\langle\zeta\rangle\right)$ and $M\left(s-\delta_{2} \lambda\langle\zeta\rangle,\langle\zeta\rangle\right)$ satisfy the inequalities (2.18) and (2.20). Hence,

$$
\begin{gathered}
\left|P^{(\tau)}\left(s-\delta_{2}\langle\zeta\rangle,\langle\zeta\rangle\right)-P^{(\tau)}(s,\langle\zeta\rangle)\right| \leqslant c \delta_{2}|\xi|\left(|s|+|\xi|^{2}\right) \\
\leqslant c \delta_{2} \frac{\left(1+\kappa^{2}\right)^{1 / 4}}{\sqrt{a}}|s|^{3 / 2}|\xi|+c \frac{\delta_{2}}{\tau} \tau|\xi|^{3} \leqslant c\left(1+\kappa^{2}\right)^{1 / 4} \max \left(\frac{\delta_{2}}{\sqrt{a}}, \frac{\delta_{2}}{\tau}\right)\left(|s|\left(|s|+|\xi|^{2}\right)+\tau|\xi|^{3}\right) .
\end{gathered}
$$

This implies (2.23), if $\delta_{2} / \sqrt{a}$ and $\delta_{2} / \tau$ are small. The proposition is proved.

PROPOSITION 2.2 The functions

$$
\begin{aligned}
& V_{1}\left(x^{\prime}, t\right)=F^{-1} L^{-1} \frac{p_{k}(\xi,|\xi|)}{P_{\varepsilon}(s,|\xi|)}, \quad k>0, \\
& V_{2}=F^{-1} L^{-1} \frac{p_{k+1}(\xi,|\xi|)}{P_{\varepsilon}(s,|\xi|)\left(r_{\varepsilon}+|\xi|\right)}, \quad k>0,
\end{aligned}
$$




$$
V_{3}\left(x^{\prime}, t\right)=F^{-1} L^{-1} \frac{M(\varepsilon s,|\xi|) p_{k-2}(\xi,|\xi|)}{P_{\varepsilon}(s,|\xi|)}, \quad k \geqslant 2,
$$

where $p_{k}$ is a homogeneous polynomial of order $k$ with respect to $\xi_{j}$, $|\xi|$, satisfy the inequalities

$$
\left|D_{x^{\prime}}^{j} V_{i}\left(x^{\prime}, t\right)\right| \leqslant \frac{c(t, j)}{\left(\left|x^{\prime}\right|+t\right)^{k+|j|}}
$$

in which $c(t, j)$ are increasing functions of $t$ independent of $\varepsilon$.

Proof. Since the number $k$ can be arbitrarily large, it suffices to prove (2.28) for $j=0$. We consider the function $V_{1}\left(x^{\prime}, t\right), t>0$. Using the Cauchy theorem, we change the contour of integration with respect to $s$ in the formula (2.13) for $V_{1}$ replacing the line $\operatorname{Re} s=a>0$ with

$$
\ell(a)=\{\operatorname{Re} s=a-\kappa|\operatorname{Im} s|\}
$$

and we introduce new variables $\xi^{\prime}=\sqrt{\frac{t}{\varepsilon}} \xi, \quad s^{\prime}=t s$. This gives

$$
V_{1}\left(x^{\prime}, t\right)=\frac{1}{(2 \pi)^{2} 2 \pi i}\left(\frac{\varepsilon}{t}\right)^{\frac{k}{2}} \int_{\mathbb{R}^{2}} e^{i y^{\prime} \cdot \xi^{\prime}} p_{k}\left(\xi^{\prime},\left|\xi^{\prime}\right|\right) \mathrm{d} \xi^{\prime} \int_{\ell(a)} e^{s^{\prime}} \frac{\mathrm{d} s^{\prime}}{P^{(\tau)}\left(s^{\prime},\left|\xi^{\prime}\right|\right)}
$$

where $y^{\prime}=x^{\prime}\left(\frac{\varepsilon}{t}\right)^{1 / 2}, \tau=\sqrt{\varepsilon t}$. The number $a>0$ is arbitrary because the parallel shift of the contour $\ell(a)$ does not change the value of the integral. We set

$$
a=1+K \varepsilon t \equiv a(t), \quad K \gg 1,
$$

and, taking account of Proposition 2.2, shift the contour $\ell(a(t))$ to the left by $\delta_{3} \tau|\xi|, \delta_{3} \ll 1$, to obtain

$$
V_{1}\left(x,{ }^{\prime} t\right)=\frac{1}{(2 \pi)^{3} i}\left(\frac{\varepsilon}{t}\right)^{\frac{k}{2}} \int_{\mathbb{R}^{2}} e^{i y^{\prime} \cdot \xi^{\prime}} p_{k}(\xi,|\xi|) e^{-\delta_{3} \tau\left|\xi^{\prime}\right|} \mathrm{d} \xi \int_{\ell(a(t))} \frac{e^{s} \mathrm{~d} s}{P^{(\tau)}\left(s-\delta_{3} \tau\left|\xi^{\prime}\right|,\left|\xi^{\prime}\right|\right)}
$$

Finally, assuming that $y_{1}=y_{2}=|y| / \sqrt{2}$ (which can be achieved by rotation of coordinate axes), we deform the contours of integration with respect to $\xi_{1}$ and $\xi_{2}$ (i.e. $\mathbb{R}$ ) into the contours $\zeta_{j}=$ $\xi_{j}+i \delta_{1}\left|\xi_{j}\right|, \quad j=1,2$, with a small $\delta_{1}$. This is possible due to the Jordan lemma, because the integrand decays at infinity and

$$
\operatorname{Re}\left(i y_{j} \zeta_{j}\right)=-\frac{|y|}{\sqrt{2}} \eta_{j}<0 \quad \text { for } \quad \eta_{j}>0,
$$

and this leads to

$V_{1}\left(x^{\prime}, t\right)=\frac{1+\delta_{1}^{2}}{(2 \pi)^{3} i}\left(\frac{\varepsilon}{t}\right)^{\frac{k}{2}} \int_{\mathbb{R}^{2}} e^{i y^{\prime} \cdot \xi^{\prime}-\frac{\delta_{1}\left|y^{\prime}\right|}{\sqrt{2}} \sum_{j}\left|\xi_{j}\right|-\delta_{3} \tau\langle\zeta\rangle} p_{k}(\zeta,\langle\zeta\rangle) \mathrm{d} \xi \int_{\ell(a(t))} \frac{e^{s} \mathrm{~d} s}{P^{(\tau)}\left(s-\delta_{3} \tau\langle\zeta\rangle,\langle\zeta\rangle\right)}$

(we observe that this device was used in [1]). Since, in virtue of (2.23),

$$
\left|P^{(\tau)}\left(s-\delta_{3} \tau\langle\zeta\rangle,\langle\zeta\rangle\right)\right| \geqslant c\left(|s|\left(|s|+|\xi|^{2}\right)+\tau|\xi|^{3}\right) \geqslant c|\xi|^{2}
$$


we have

$$
\begin{aligned}
\left|V_{1}\left(x^{\prime}, t\right)\right| & \leqslant \frac{c \varepsilon^{\frac{k}{2}}}{t^{\frac{k}{2}}} \int_{\mathbb{R}^{2}} e^{-c\left(\left|y^{\prime}\right|+\sqrt{\varepsilon t}\right)|\xi|}|\xi|^{k-2} \mathrm{~d} \xi \int_{0}^{\infty} e^{a(t)-\kappa r} \mathrm{~d} r \\
& \leqslant \frac{c \varepsilon^{\frac{k}{2}}}{t^{\frac{k}{2}}\left(\left|y^{\prime}\right|+\sqrt{\varepsilon t}\right)^{k}} \leqslant \frac{c}{\left(\left|x^{\prime}\right|+t\right)^{k}} .
\end{aligned}
$$

Inequality (2.28) with $j=0$ for $V_{1}$ is proved. For $V_{2}$ it is obtained in exactly the same way. For $V_{3}$, instead of (2.29), we have

$$
\begin{aligned}
V_{3}\left(x^{\prime}, t\right)= & \frac{1+\delta_{1}^{2}}{(2 \pi)^{3} i}\left(\frac{\varepsilon}{t}\right)^{\frac{k}{2}} \int_{\mathbb{R}^{2}} e^{i y^{\prime} \cdot \xi^{\prime}-\frac{\delta_{1}\left|y^{\prime}\right|}{\sqrt{2}} \sum_{j}\left|\xi_{j}\right|-\delta_{3} \tau\langle\zeta\rangle} p_{k-2}(\zeta,\langle\zeta\rangle) \mathrm{d} \xi \\
& \int_{\ell(a(t))} \frac{M\left(s-\delta_{3} \tau\langle\zeta\rangle,\langle\zeta\rangle\right) e^{s} \mathrm{~d} s}{P^{(\tau)}\left(s-\delta_{3} \tau\langle\zeta\rangle,\langle\zeta\rangle\right)}
\end{aligned}
$$

so, using the inequality $|M| /\left|P^{(\tau)}\right| \leqslant c|s|^{-1} \leqslant c$, we arrive at the same estimate (2.28).

If $t<0$, then, by the Jordan lemma,

$$
\int_{\operatorname{Re} s=a} e^{s t} \frac{\mathrm{d} s}{P_{\varepsilon}(s,|\xi|)}=0, \quad \int_{\operatorname{Re} s=a} e^{s t} \frac{M(s,|\xi|) \mathrm{d} s}{P_{\varepsilon}(s,|\xi|)}=0,
$$

hence, $V_{1}\left(x^{\prime}, t\right)=V_{2}\left(x^{\prime}, t\right)=V_{3}\left(x^{\prime}, t\right)=0$. The proposition is proved.

COROLLARY 2.1 If $k=2$, then the kernels $V_{i}$ satisfy the inequalities

$$
\int_{0}^{t} \mathrm{~d} \tau \int_{\mathbb{R}^{2}}\left|\Delta_{j}^{m}(h) V_{i}\left(x^{\prime}, \tau\right)\right| \leqslant c(t) h, \quad m>1,
$$

where

$$
\Delta_{j}^{m}(h) V_{i}\left(x^{\prime}, \tau\right)=\sum_{k=0}^{m}(-1)^{m-k} C_{m}^{k} V_{i}\left(x^{\prime}+e_{j} k h, \tau\right)
$$

is a finite difference of $V_{i}$ with respect to $x_{j}$ of order $m, e_{1}=(1.0), e_{2}=(0,1)$.

Indeed, we have

$$
\begin{gathered}
\int_{0}^{t} \mathrm{~d} \tau \int_{\mathbb{R}^{2}}\left|\Delta_{j}^{m}(h) V_{i}\left(x^{\prime}, \tau\right)\right| \mathrm{d} x^{\prime} \leqslant \sum_{k=0}^{m} C_{m}^{k} \int_{0}^{t} \mathrm{~d} \tau \int_{\left|x^{\prime}\right|<2 m h}\left|V_{i}\left(x^{\prime}+e_{j} k h, \tau\right)\right| \mathrm{d} x^{\prime} \\
\quad+\int_{0}^{t} \mathrm{~d} \tau \int_{\left|x^{\prime}\right|>2 m h}\left|\int_{0}^{h} \ldots \int_{0}^{h} \frac{\partial^{m} V_{i}\left(x+e_{j}\left(t_{1}+\ldots t_{m}\right)\right)}{\partial x_{j}^{m}} \mathrm{~d} t_{1} \ldots \mathrm{d} t_{m}\right| \\
\leqslant c \sum_{k=0}^{m} \int_{0}^{t} \mathrm{~d} \tau \int_{\left|x^{\prime}\right|<2 m h} \frac{\mathrm{d} x^{\prime}}{\left(\left|x^{\prime}+e_{j} k h\right|+\tau\right)^{2}}+c \int_{0}^{t} \mathrm{~d} \tau \int_{\left|x^{\prime}\right|>2 m h} \frac{h^{m} \mathrm{~d} x^{\prime}}{\left(\left|x^{\prime}\right|+\tau\right)^{2+m}} \leqslant c h .
\end{gathered}
$$


2.3 Estimates of solutions of Problems (2.1), (2.2), and (2.15)

THEOREM 2.1 Assume that $b_{1}, b_{2}$ are smooth functions decaying like power functions as $\left|x^{\prime}\right| \rightarrow$ $\infty$ together with their derivatives and satisfying the conditions

$$
b_{1}\left(x^{\prime}, 0\right)=b_{2}\left(x^{\prime}, 0\right)=0 .
$$

Further, let

$$
b_{3}^{\prime}\left(x^{\prime}, t\right)=b_{3}\left(x^{\prime}, t\right)+\int_{0}^{t} B\left(x^{\prime}, \tau\right) \mathrm{d} \tau
$$

with $b_{3}, B$ possessing the same properties. Then the solution of problem (2.1)-(2.2) constructed in Subsection 2.1 satisfies the inequality

$$
\begin{aligned}
\varepsilon \sup _{\tau<t}\left[\mathbf{v}_{\tau}(\cdot, \tau)\right]_{\mathbb{R}_{+}^{3}}^{(\alpha)}+\sup _{\tau<t}[\mathbf{v}(\cdot, \tau)]_{\mathbb{R}_{+}^{3}}^{(2+\alpha)}+\sup _{\tau<t}[p(\cdot, \tau)]_{\mathbb{R}_{+}^{3}}^{(1+\alpha)} \leqslant \\
\leqslant c\left(\sum_{k=1}^{m} \sup _{\tau<t}\left[b_{k}(\cdot, \tau)\right]_{\mathbb{R}^{2}}^{(1+\alpha)}+\varepsilon^{\frac{1+\alpha}{2}} \sum_{j=1}^{2} \sup _{\mathbb{R}^{2}}\left[b_{j}\left(x^{\prime}, \cdot\right)\right]_{(0, t)}^{\left(\frac{1+\alpha}{2}\right)}+\sup _{\tau<t}[B(\cdot, \tau)]_{\mathbb{R}^{2}}^{(\alpha)}\right) .
\end{aligned}
$$

Proof. We consider the solution of problem (2.1)-(2.2) as the solutions of (2.1)-(2.6) with $a_{1}=$ $b_{1}, a_{2}=b_{2}$ and with $a_{3}$ given by formulas (2.11) and (2.14). Under our hypotheses, these formulas take the form

$\widetilde{a}_{3}=\widetilde{b}_{3}+\sum_{j=1}^{2} \frac{|\xi| i \xi_{j}}{P_{\varepsilon}(s,|\xi|)} i \xi_{j} \widetilde{b}_{3}+\frac{M(\varepsilon s,|\xi|)}{P_{\varepsilon}(s,|\xi|)} \widetilde{B}-\left(\frac{|\xi|^{2}}{P_{\varepsilon}(s,|\xi|)}-\frac{2|\xi|^{3}}{P_{\varepsilon}(s,|\xi|)\left(r_{\varepsilon}+|\xi|\right)}\right) \sum_{j=1}^{2} i \xi_{j} \widetilde{b}_{j}$

or

$$
\begin{aligned}
a_{3}\left(x^{\prime}, t\right) & =\sum_{j=1}^{2} \int_{0}^{t} \mathrm{~d} \tau \int_{\mathbb{R}^{2}} W\left(x^{\prime}-y^{\prime}, t-\tau\right) \frac{\partial b_{j}\left(y^{\prime}, \tau\right)}{\partial y_{j}} \mathrm{~d} y^{\prime} \\
& +\sum_{j=1}^{2} \int_{0}^{t} \mathrm{~d} \tau \int_{\mathbb{R}^{2}} W_{j}\left(x^{\prime}-y^{\prime}, t-\tau\right) \frac{\partial b_{3}\left(y^{\prime}, \tau\right)}{\partial y_{j}} \mathrm{~d} y^{\prime}+b_{3}\left(x^{\prime}, t\right) \\
& +\int_{0}^{t} \mathrm{~d} \tau \int_{\mathbb{R}^{2}} V\left(x^{\prime}-y^{\prime}, t-\tau\right) B\left(y^{\prime}, \tau\right) \mathrm{d} y^{\prime}
\end{aligned}
$$

with the kernels $W, W_{j}, V$ defined by

$$
\begin{gathered}
W=-F^{-1} L^{-1}\left(\frac{|\xi|^{2}}{P_{\varepsilon}(s,|\xi|)}-\frac{2|\xi|^{3}}{P_{\varepsilon}(s,|\xi|)\left(r_{\varepsilon}+|\xi|\right)}\right), \\
W_{j}=F^{-1} L^{-1} \frac{i \xi_{j}|\xi|}{P_{\varepsilon}(s,|\xi|)}, \quad V=F^{-1} L^{-1} \frac{M(\varepsilon s,|\xi|)}{P_{\varepsilon}(s,|\xi|)} .
\end{gathered}
$$

All these kernels satisfy inequalities (2.28) and (2.30), so the integrals in (2.32) are convergent, if $\nabla b_{k}, B$ decay at infinity like power functions. Moreover, from (2.30) and from a general result due to K. K. Golovkin (see [2, 3]) it follows that

$$
\sup _{\tau<t}\left[a_{3}(\cdot, \tau)\right]_{\mathbb{R}^{2}}^{(1+\alpha)} \leqslant c\left(\sum_{k=1}^{3} \sup _{\tau<t}\left[b_{k}(\cdot, \tau)\right]_{\mathbb{R}^{2}}^{(1+\alpha)}+\sup _{\tau<t}[B(\cdot, \tau)]_{\mathbb{R}^{2}}^{(\alpha)}\right) .
$$


Solutions of the initial-boundary value problem for the Stokes equations with boundary conditions (2.6) were estimated in the paper [18]; in particular, Theorem 4.1 of this paper yields the following inequality for the solution of Problem (2.1) and (2.6):

$$
\begin{aligned}
\varepsilon \sup _{\tau<t}\left[\mathbf{v}_{\tau}(\cdot, \tau)\right]_{\mathbb{R}_{+}^{3}}^{(\alpha)}+\sup _{\tau<t}[\mathbf{v}(\cdot, \tau)]_{\mathbb{R}_{+}^{3}}^{(2+\alpha)}+\sup _{\tau<t}[p(\cdot, \tau)]_{\mathbb{R}_{+}^{3}}^{(1+\alpha)} & \\
& \leqslant c\left(\sum_{k=1}^{3} \sup _{\tau<t}\left[a_{k}(\cdot, \tau)\right]_{\mathbb{R}^{2}}^{(1+\alpha)}+\varepsilon^{\frac{1+\alpha}{2}} \sum_{j=1}^{2} \sup _{\mathbb{R}^{2}}\left[a_{j}\left(x^{\prime}, \cdot\right)\right]_{(0, t)}^{\left(\frac{1+\alpha}{2}\right)}\right) .
\end{aligned}
$$

Estimate (2.31) follows from (2.33) and (2.34). The theorem is proved.

THEOREM 2.2 Let $\mathbf{f}, g, \mathbf{v}_{0}, b_{1}, b_{2}$ be smooth functions with compact supports defined for $t \in$ $(0, T)$ and satisfying the compatibility conditions

$$
\nabla \cdot \mathbf{v}_{0}(x)=g(x, 0), \quad \frac{\partial v_{0 j}}{\partial x_{3}}+\left.\frac{\partial v_{03}}{\partial x_{j}}\right|_{x_{3}=0}=b_{j}\left(x^{\prime}, 0\right), \quad j=1,2
$$

moreover, let

$$
b_{3}^{\prime}\left(x^{\prime}, t\right)=b_{3}\left(x^{\prime}, t\right)+\int_{0}^{t} B\left(x^{\prime}, \tau\right) \mathrm{d} \tau \quad g(x, t)=\nabla \cdot \mathbf{h}(x, t)+h_{0}(x, t)
$$

where $b_{3}, B, \mathbf{h}, h_{0}$ are also smooth and have compact supports. Then Problem (2.15) has a unique solution in a class of bounded continuous functions with a finite norm

$$
Y_{T}(\mathbf{v}, p)=\varepsilon \sup _{t<T}\left[\mathbf{v}_{t}(\cdot, t)\right]_{\mathbb{R}_{+}^{3}}^{(\alpha)}+\sup _{t<T}\left[\mathbf{v}_{t}(\cdot, t)\right]_{\mathbb{R}_{+}^{3}}^{(2+\alpha)}+\sup _{t<T}[p(\cdot, t)]_{\mathbb{R}_{+}^{3}}^{(1+\alpha)},
$$

and for arbitrary $t<T$

$$
\begin{gathered}
Y_{t}(\mathbf{v}, p) \leqslant c\left(\sup _{\tau<t}[\mathbf{f}(\cdot, \tau)]_{\mathbb{R}_{+}^{3}}^{(\alpha)}+\sup _{\tau<t}[g(\cdot, \tau)]_{\mathbb{R}_{+}^{3}}^{(1+\alpha)}+\left[\mathbf{v}_{0}\right]_{\mathbb{R}_{+}^{3}}^{(2+\alpha)}+\varepsilon \sup _{\tau<t}\left[\mathbf{h}_{\tau}(\cdot, \tau)\right]_{\mathbb{R}_{+}^{3}}^{(\alpha)}\right. \\
+\varepsilon D^{1-\alpha} \sup _{\tau<t} \sup _{\mathbb{R}_{+}^{3}}\left|h_{0 \tau}(x, \tau)\right|+\sum_{k=1}^{3} \sup _{\tau<t}\left[b_{k}(\cdot, \tau)\right]_{\mathbb{R}^{2}}^{(1+\alpha)} \\
\left.+\varepsilon^{\frac{1+\alpha}{2}} \sum_{j=1}^{2} \sup _{\mathbb{R}^{2}}\left[b_{j}\left(x^{\prime}, \cdot\right)\right]_{(0, t)}^{\left(\frac{1+\alpha}{2}\right)}+\sup _{\tau<t}[B(\cdot, \tau)]_{\mathbb{R}^{2}}^{(\alpha)}\right) \equiv F_{t}
\end{gathered}
$$

where $D=\sup _{\tau<T}\left(\operatorname{diam} \operatorname{supp}_{0}(x, \tau)\right)$.

Proof. We obtain the estimate (2.36) for the solution of Problem (2.15) constructed above. If the extension of $\mathbf{f}$ and $\mathbf{v}_{0}$ into the domain $x_{3}<0$ in (2.16) is made in such a way that

$$
\left[\mathbf{f}^{*}(\cdot, t)\right]_{\mathbb{R}^{3}}^{(\alpha)} \leqslant c[\mathbf{f}(\cdot, t)]_{\mathbb{R}_{+}^{3}}^{(\alpha)}, \quad\left[\mathbf{v}_{0}^{*}\right]_{\mathbb{R}^{3}}^{(2+\alpha)} \leqslant c\left[\mathbf{v}_{0}\right]_{\mathbb{R}_{+}^{3}}^{(2+\alpha)},
$$

then, as it follows from the estimates (4.9) and (4.11) in [18],

$$
\sum_{j=1}^{2}\left(\varepsilon \sup _{\tau<t}\left[\mathbf{v}_{\tau}^{(j)}(\cdot, \tau)\right]_{\mathbb{R}_{+}^{3}}^{(\alpha)}+\sup _{\tau<t}\left[\mathbf{v}^{(j)}(\cdot, \tau)\right]_{\mathbb{R}_{+}^{3}}^{(2+\alpha)}\right)+\sup _{\tau<t}\left[p^{(2)}(\cdot, \tau)\right]_{\mathbb{R}_{+}^{3}}^{(1+\alpha)}
$$




$$
\begin{aligned}
& \leqslant c\left(\sup _{\tau<t}[\mathbf{f}(\cdot, \tau)]_{\mathbb{R}_{+}^{3}}^{(\alpha)}+\sup _{\tau<t}[g(\cdot, \tau)]_{\mathbb{R}_{+}^{3}}^{(1+\alpha)}+\left[\mathbf{v}_{0}\right]_{\mathbb{R}_{+}^{3}}^{(2+\alpha)}\right. \\
& \left.+\varepsilon \sup _{\tau<t}\left[\mathbf{h}_{\tau}(\cdot, \tau)\right]_{\mathbb{R}_{+}^{3}}^{(\alpha)}+\varepsilon D^{1-\alpha} \sup _{\tau<t} \sup _{\mathbb{R}_{+}^{3}}\left|h_{0 \tau}(x, \tau)\right|\right) .
\end{aligned}
$$

Finally, applying Theorem 2.1 to Problem (2.17), we obtain

$$
\begin{aligned}
& Y_{t}\left(\mathbf{v}^{(3)}, p^{(3)}\right) \leqslant c\left(\sum_{k=1}^{3} \sup _{\tau<t}\left[b_{k}(\cdot, \tau)\right]_{\mathbb{R}^{2}}^{(1+\alpha)}+\varepsilon^{\frac{1+\alpha}{2}} \sum_{j=1}^{2} \sup _{\mathbb{R}^{2}}\left[b_{j}\left(x^{\prime}, \cdot\right)\right]_{(0, t)}^{\left(\frac{1+\alpha}{2}\right)}+\sup _{\tau<t}[B(\cdot, \tau)]_{\mathbb{R}^{2}}^{(\alpha)}\right. \\
& \left.\quad+\sum_{j=1}^{2} \sup _{\tau<t}\left[\mathbf{v}_{\tau}^{(j)}(\cdot, \tau)\right]_{\mathbb{R}_{+}^{3}}^{(2+\alpha)}+\sup _{\tau<t}\left[p^{(2)}(\cdot, \tau)\right]_{\mathbb{R}_{+}^{3}}^{(1+\alpha)}+\varepsilon^{\frac{1+\alpha}{2}} \sum_{j=1}^{2} \sup _{\mathbb{R}^{2}}\left[\nabla \mathbf{v}^{(j)}\left(x^{\prime}, \cdot\right)\right]_{(0, t)}^{\left(\frac{1+\alpha}{2}\right)}\right) .
\end{aligned}
$$

But the last term, in virtue of interpolation inequality

$$
\varepsilon^{\frac{1+\alpha}{2}} \sup _{\mathbb{R}^{2}}\left[\nabla u\left(x^{\prime}, \cdot\right)\right]_{(0, t)}^{\left(\frac{1+\alpha}{2}\right)} \leqslant c\left(\varepsilon \sup _{\tau<t}\left[u_{\tau}^{(j)}(\cdot, \tau)\right]_{\mathbb{R}_{+}^{3}}^{(\alpha)}+\sup _{\tau<t}[u(\cdot, \tau)]_{\mathbb{R}_{+}^{3}}^{(2+\alpha)}\right)
$$

(see $[6,18])$ can be estimated by the left-hand side of $(2.37)$. Hence, putting all the estimates together, we arrive at (2.36).

The uniqueness of the solution of Problem (2.15) can be proved in the same way as in [18]. Let $(\mathbf{w}, s)$ be a bounded continuous solution of a homogeneous problem with a finite norm (2.35) and let $\zeta \in C_{0}^{\infty}\left(\mathbb{R}^{3}\right)$ be a function equal to one for $|x|<1$ and to zero for $|x|>2$. The functions $\mathbf{W}_{R}=\zeta_{R} \mathbf{W}, S_{R}=\zeta_{R} s$ where $\zeta_{R}(x)=\zeta(x / R)$ satisfy the equations (2.15) with

$$
\begin{gathered}
\mathbf{f}=-2 \nabla \mathbf{w} \nabla \zeta_{R}-\mathbf{w} \nabla^{2} \zeta_{R}+s \nabla \zeta_{R}, \quad g=\nabla \zeta_{R} \cdot \mathbf{w} \equiv h_{0}, \\
b_{j}=w_{3} \frac{\partial \zeta_{R}}{\partial x_{j}}+\left.w_{j} \frac{\partial \zeta_{R}}{\partial x_{3}}\right|_{x_{3}=0}, \quad b_{3}=\left.2 w_{3} \frac{\partial \zeta_{R}}{\partial x_{3}}\right|_{x_{3}=0}, \quad B=\left.\left(\nabla^{\prime} w_{3} \cdot \nabla^{\prime} \zeta_{R}+w_{3} \nabla^{\prime 2} \zeta_{R}\right)\right|_{x_{3}=0} .
\end{gathered}
$$

Clearly, $\mathbf{w}_{R}, s_{R}$ are expressed in terms of these functions as indicated above. Since $\mathbf{w}, s$ and their derivatives are bounded, estimate (2.36) gives

$$
Y_{t}\left(\mathbf{W}_{R}, S_{R}\right) \leqslant c R^{-\alpha} \rightarrow 0, \quad \text { as } R \rightarrow \infty .
$$

Hence, $\mathbf{w}=0, s=0$, q.e.d. The theorem is proved.

\section{Proof of Theorem 5}

We start with the proof of the estimate (1.23) by a standard method of Schauder; the proof is very close to that of inequality (1.3) in [18]. We estimate the solution in a small neighbourhood of an arbitrary point $x_{0} \in \bar{\Omega}$ assuming (without loss of generality) that $x_{0}=0$ and that $\mathrm{O} x_{3}$-axis is directed along the interior normal $-\mathbf{n}_{0}\left(x_{0}\right)$.

Let

$$
x_{3}=\phi\left(x^{\prime}\right), \quad x^{\prime} \in \mathcal{B}_{d}=\left\{\left|x^{\prime}\right|<d\right\}, \quad \phi \in C^{2+\alpha}\left(\mathcal{B}_{d}\right),
$$


be the equation of the surface $\Gamma$ in the neighbourhood of the point $x_{0}=0$. Then the normal and the Laplace-Beltrami operator on $\Gamma$ are defined by

$$
\mathbf{n}_{0}(x)=\left(\frac{\phi_{x_{1}}}{\sqrt{1+\left|\nabla \phi\left(x^{\prime}\right)\right|^{2}}}, \frac{\phi_{x_{2}}}{\sqrt{1+\left|\nabla \phi\left(x^{\prime}\right)\right|^{2}}},-\frac{1}{\sqrt{1+\left|\nabla \phi\left(x^{\prime}\right)\right|^{2}}}\right)
$$

and

$$
\Delta_{0} f=\frac{1}{\sqrt{g^{(0)}}} \sum_{\alpha, \beta=1}^{2} \frac{\partial}{\partial x_{\alpha}}\left(\frac{\widehat{g}_{\alpha \beta}^{(0)}}{\sqrt{g^{(0)}}} \frac{\partial f}{\partial x_{\beta}}\right)
$$

where $g^{(0)}=\operatorname{det}\left(g_{\alpha \beta}^{(0)}\right)_{\alpha, \beta=1,2}$,

$$
g_{\alpha \beta}^{(0)}=\frac{\partial \mathbf{r}}{\partial x_{\alpha}} \cdot \frac{\partial \mathbf{r}}{\partial x_{\beta}}=\delta_{\alpha \beta}+\phi_{x_{\alpha}} \phi_{x_{\beta}}, \quad \mathbf{r}=\left(x_{1}, x_{2}, \phi\left(x^{\prime}\right)\right),
$$

and $\widehat{g}_{\alpha \beta}^{(0)}$ are elements of associated matrix to $\left(g_{\alpha \beta}^{(0)}\right)_{\alpha, \beta=1,2}$, i.e.

$$
\widehat{g}_{11}^{(0)}=g_{22}^{(0)}, \quad \widehat{g}_{22}^{(0)}=g_{11}^{(0)}, \quad \widehat{g}_{12}^{(0)}=\widehat{g}_{21}^{(0)}=-g_{12}^{(0)} .
$$

We 'rectify' $\Gamma$ near the origin by the transformation $y=Z(x)$ :

$$
y_{1}=x_{1}, \quad y_{2}=x_{2}, \quad y_{3}=x_{3}-\phi\left(x^{\prime}\right), \quad x^{\prime} \in K_{d}^{\prime},
$$

and we set

$$
\mathbf{u}=\zeta_{r} \mathbf{v}, \quad q=\zeta_{r} p
$$

where $\zeta_{r}(y)=\zeta(y / r)$ and $\zeta(y)$ is the same function as in Theorem 2.2. By virtue of (1.21) and (1.22),

$$
\begin{aligned}
& \varepsilon \mathbf{u}_{t}-\nabla^{2} \mathbf{u}+\nabla q=\zeta_{r} \mathbf{f}_{1}+\mathbf{f}^{\prime}+\mathbf{m}_{1}(\mathbf{u}, q), \\
& \nabla \cdot \mathbf{u}=g \zeta_{r}+g^{\prime}+m_{2}(\mathbf{u}),\left.\quad \mathbf{u}\right|_{t=0}=0, \\
& \left.S_{i 3}(\mathbf{u})\right|_{y_{3}=0}=-\left(\zeta_{r} b_{i}+b_{i}^{\prime}\right)+m_{3 i}(\mathbf{u}), \quad i=1,2, \\
& T_{33}(\mathbf{u}, q)+\left.\int_{0}^{t} \Delta^{\prime} u_{3}\left(y^{\prime}, \tau\right) \mathrm{d} \tau\right|_{y_{3}=0}=b \zeta_{r}+b^{\prime}+m_{4}(\mathbf{u})+\int_{0}^{t}\left(B \zeta_{r}+B^{\prime}+m_{5}(\mathbf{u})\right) \mathrm{d} \tau, \\
& \mathbf{f}^{\prime}=-2 \widehat{\nabla} \mathbf{v} \widehat{\nabla} \zeta_{r}-\mathbf{v} \widehat{\nabla}^{2} \zeta_{r}+p \widehat{\nabla} \zeta_{r}, \\
& g^{\prime}=\mathbf{v} \cdot \widehat{\nabla} \zeta_{r}, \\
& b_{i}^{\prime}=\left(\frac{\partial \zeta_{r}}{\partial y_{i}}-\phi_{y_{i}} \frac{\partial \zeta_{r}}{\partial y_{3}}\right)\left(\mathbf{v} \cdot \mathbf{n}_{0}\right)+\left(\widehat{\nabla} \zeta_{r} \cdot \mathbf{n}_{0}\right) v_{i}-\left.2 n_{i}\left(\widehat{\nabla} \zeta_{r} \cdot \mathbf{n}_{0}\right)\left(\mathbf{v} \cdot \mathbf{n}_{0}\right)\right|_{y_{3}=0}, \quad i=1,2, \\
& b^{\prime}=\left.2\left(\widehat{\nabla} \zeta_{r} \cdot \mathbf{n}_{0}\right)\left(\mathbf{v} \cdot \mathbf{n}_{0}\right)\right|_{y_{3}=0}, \\
& B^{\prime}=\left.\mathbf{n}_{0} \cdot\left(\Delta\left(\zeta_{r} \mathbf{v}\right)-\zeta_{r} \Delta \mathbf{v}\right)\right|_{y_{3}=0},
\end{aligned}
$$




$$
\begin{aligned}
& \mathbf{m}_{1}(\mathbf{u}, q)=-v\left(\nabla^{2}-\widehat{\nabla}^{2}\right) \mathbf{u}+(\nabla-\widehat{\nabla}) q, \\
& m_{2}(\mathbf{u})=(\nabla-\widehat{\nabla}) \cdot \mathbf{u}, \\
& m_{3 i}(\mathbf{u})=\left(S_{i 3}(\mathbf{u})+\left(\widehat{S}(\mathbf{u}) \mathbf{n}_{0}\right)_{i}\right)-\left.n_{0 i}\left(\mathbf{n}_{0} \cdot \widehat{S}(\mathbf{u}) \mathbf{n}_{0}\right)\right|_{y_{3}=0}, \\
& m_{4}(\mathbf{u})=\left.2\left(S_{33}(\mathbf{u})-\mathbf{n}_{0} \cdot \widehat{S}(\mathbf{u}) \mathbf{n}_{0}\right)\right|_{y_{3}=0}, \\
& m_{5}(\mathbf{u})=\left.\left(\Delta^{\prime} u_{3}+\mathbf{n}_{0} \cdot \Delta \mathbf{u}\right)\right|_{y_{3}=0}
\end{aligned}
$$

We observe that all the functions in (3.1) vanish for $|y|>2 r$, and the leading coefficients in the expressions (3.2) vanish at the origin. Hence, inequality (2.36) implies

$$
\begin{aligned}
& \varepsilon \sup _{\tau<t}\left[\mathbf{u}_{\tau}(\cdot, \tau)\right]_{\mathbb{R}_{+}^{3}}^{(\alpha)}+\sup _{\tau<t}[\mathbf{u}(\cdot, \tau)]_{\mathbb{R}_{+}^{3}}^{(2+\alpha)}+\sup _{\tau<t}[q(\cdot, \tau)]_{\mathbb{R}_{+}^{3}}^{(1+\alpha)} \\
\leqslant & c r\left(\varepsilon \sup _{\tau<t}\left[\mathbf{u}_{\tau}(\cdot, \tau)\right]_{\mathbb{R}_{+}^{3}}^{(\alpha)}+\sup _{\tau<t}[\mathbf{u}(\cdot, \tau)]_{\mathbb{R}_{+}^{3}}^{(2+\alpha)}+\sup _{\tau<t}[q(\cdot, \tau)]_{\mathbb{R}_{+}^{3}}^{(1+\alpha)}\right) \\
& +c\left(\sup _{\tau<t}\left[\zeta_{r} \mathbf{f}_{1}(\cdot, \tau)\right]_{\mathbb{R}_{+}^{3}}^{(\alpha)}+\sup _{\tau<t}\left[\zeta_{r} g(\cdot, \tau)\right]_{\mathbb{R}_{+}^{3}}^{(1+\alpha)}+\varepsilon \sup _{\tau<t}\left[\zeta_{r} \mathbf{h}_{t}(\cdot, \tau)\right]_{\mathbb{R}_{+}^{3}}^{(\alpha)}+\varepsilon \sup _{\tau<t} \sup _{\mathbb{R}_{+}^{3}}\left|\zeta_{r} h_{0 t}(y, \tau)\right|\right. \\
& \left.+\sum_{i=1}^{2} \sup _{\tau<t}\left[\zeta_{r} b_{i}(\cdot, \tau)\right]_{\mathbb{R}^{2}}^{(1+\alpha)}+\sup _{\tau<t}\left[\zeta_{r} b(\cdot, \tau)\right]_{\mathbb{R}^{2}}^{(1+\alpha)}+\varepsilon^{\frac{1+\alpha}{2}} \sum_{i=1}^{2} \sup _{\mathbb{R}^{2}}\left[\zeta_{r} b_{i}\right]_{(0, t)}^{\left(\frac{1+\alpha}{2}\right)}+\sup _{\tau<t}\left[\zeta_{r} B(\cdot, \tau)\right]_{\mathbb{R}^{2}}^{(\alpha)}\right) \\
& +c(r)\left(\sum_{0 \leqslant|j| \leqslant 2} \sup _{\tau<t} \sup _{\Omega}\left|\mathcal{D}^{j} \mathbf{v}\right|+\sup _{\tau<t} \sup _{\Omega}|\nabla p(x, \tau)|+\sup _{\tau<t} \sup _{\Omega}|p(x, t)|\right. \\
& \left.+\varepsilon \sup _{\tau<t} \sup _{\Omega}\left|\mathbf{v}_{\tau}(x, \tau)\right|+\varepsilon^{\frac{1+\alpha}{2}} \sup _{\Gamma}[\mathbf{v}(x, \cdot)]_{(0, t)}^{\left(\frac{1+\alpha}{2}\right)}\right) .
\end{aligned}
$$

Choosing $r$ so small that $c r \leqslant \frac{1}{2}$, we eliminate the first three terms in the right-hand side. A similar estimate can be obtained in the case $\operatorname{dist}\left(x_{0}, \Gamma\right)>r$. Combination of these two cases gives the inequality

$$
\begin{aligned}
Y(t) & \equiv \varepsilon \sup _{\tau<t}\left|\mathbf{v}_{\tau}(\cdot, \tau)\right|_{C^{\alpha}(\Omega)}+\sup _{\tau<t}|\mathbf{v}(\cdot, \tau)|_{C^{2+\alpha}(\Omega)}+\sup _{\tau<t}|p(\cdot, \tau)|_{C^{1+\alpha}(\Omega)} \\
& \leqslant c\left(N(t)+Y_{0}(t)\right),
\end{aligned}
$$

where $N(t)$ is the sum of norms in the right-hand side of (1.23) and

$$
Y_{0}(t)=\sum_{0 \leqslant|j| \leqslant 2} \sup _{\tau<t} \sup _{\Omega}\left|D_{x}^{j} \mathbf{v}(x, t)\right|+\sup _{\tau<t} \sup _{\Omega}|\nabla p(x, \tau)|+\sup _{\tau<t} \sup _{\Omega}|p(x, t)| .
$$

By virtue of interpolation inequalities,

$$
Y_{0}(t) \leqslant \varepsilon_{1} Y(t)+c\left(\varepsilon_{1}\right)\left(\sup _{\tau<t}\|\mathbf{v}\|_{L_{2}(\Omega)}+\sup _{\tau<t} \sup _{\Omega}|p(x, t)|\right)
$$


where $\varepsilon_{1}$ is an arbitrarily small positive number. The function $p(x, t)$ can be considered as a solution of the Dirichlet problem

$$
\begin{aligned}
& \nabla^{2} p=\nabla \cdot \mathbf{f}+\nabla^{2} g-\varepsilon\left(\nabla \cdot \mathbf{h}_{t}+h_{0 t}\right), \quad x \in \Omega, \\
& \left.p\right|_{\Gamma}=\left.\left(2 \mathbf{n}_{0} \cdot \frac{\partial \mathbf{v}}{\partial n_{0}}-\mathbf{n}_{0} \cdot \int_{0}^{t} \Delta_{0} \mathbf{v} \mathrm{d} \tau\right)\right|_{\Gamma}-b-\int_{0}^{t} B \mathrm{~d} \tau,
\end{aligned}
$$

and it satisfies the inequality

$$
\begin{aligned}
& \sup _{\Omega}|p(x, t)| \leqslant c\left(\sup _{\Omega}|\mathbf{f}(x, t)|+\sup _{\Omega}|\nabla g(x, t)|+\varepsilon\left(\sup _{\Omega}\left|\mathbf{h}_{t}(x, t)\right|+\sup _{\Omega}\left|h_{0 t}(x, t)\right|\right)\right. \\
& \left.+\sup _{\Gamma}|b(x, t)|+\int_{0}^{t} \sup _{\Gamma}|B(x, \tau)| \mathrm{d} \tau+\sup _{\Omega}|\nabla \mathbf{v}(x, t)|+\int_{0}^{t} \sup _{\Gamma}\left|\Delta_{0} \mathbf{v}(x, \tau)\right| \mathrm{d} \tau\right)
\end{aligned}
$$

(see, for instance, [10], Lemma 5.1). Finally, we have the energy relation

$$
\begin{aligned}
\frac{\varepsilon}{2} \frac{\mathrm{d}}{\mathrm{d} t}\|\mathbf{v}\|_{L_{2}(\Omega)}^{2}+\frac{1}{2} \| & S(\mathbf{v}) \|_{L_{2}(\Omega)}^{2}+\sum_{k=1}^{6}\left|\int_{\Omega} \mathbf{v} \cdot \boldsymbol{\varphi}_{k} \mathrm{~d} x\right|^{2} \\
= & \int_{\Omega}(\mathbf{f}-\nabla g) \cdot \mathbf{v} \mathrm{d} x+\int_{\Gamma}\left(\mathbf{b} \cdot \Pi_{0} \mathbf{v}+\left(b+\int_{0}^{t} B \mathrm{~d} \tau\right)\left(\mathbf{v} \cdot \mathbf{n}_{0}\right)\right) \mathrm{d} S \\
& +\int_{\Gamma}\left(\mathbf{v}(x, t) \cdot \mathbf{n}_{0}(x)\right) \mathrm{d} S \int_{0}^{t} \mathbf{n}_{0}(x) \Delta_{0} \mathbf{v}(x, \tau) \mathrm{d} \tau+\int_{\Omega} p g \mathrm{~d} x .
\end{aligned}
$$

By virtue of the Korn inequality (in the form presented in [13]),

$$
\frac{1}{2}\|S(\mathbf{v})\|_{L_{2}(\Omega)}^{2}+\sum_{k=1}^{6}\left|\int_{\Omega} \mathbf{v} \cdot \varphi_{k} \mathrm{~d} x\right|^{2} \geqslant \frac{c}{2}\|\mathbf{v}\|_{W_{2}^{1}(\Omega)}^{2},
$$

so (3.6) implies

$$
\varepsilon \frac{\mathrm{d}}{\mathrm{d} t}\|\mathbf{v}\|^{2}+c\|\mathbf{v}\|^{2} \leqslant c_{1}\left(N(t)+\int_{0}^{t} Y_{0}(\tau) \mathrm{d} \tau\right)^{2}
$$

and

$$
\begin{aligned}
\|\mathbf{v}(\cdot, t)\|^{2} & \leqslant \frac{c_{1}}{\varepsilon} \int_{0}^{t} e^{-\frac{c}{\varepsilon}(t-\tau)}\left(N(\tau)+\int_{0}^{\tau} Y_{0}\left(\tau^{\prime}\right) \mathrm{d} \tau^{\prime}\right)^{2} \mathrm{~d} \tau \\
& \leqslant \frac{c_{1}}{c}\left(\sup _{\tau<t} N(\tau)+\int_{0}^{t} Y_{0}(\tau) \mathrm{d} \tau\right)^{2} .
\end{aligned}
$$

From this inequality and from (3.4)-(3.6) it follows that

$$
Y_{0}(t) \leqslant c\left(N(t)+\int_{0}^{t} Y_{0}(\tau) \mathrm{d} \tau\right),
$$

$Y_{0}(t) \leqslant c N(t)$, and finally, taking (3.3) into account we arrive at (1.23). 
As for the solvability of problem (1.21)-(1.22), we prove it making use of Theorem 6 . This gives us the possibility to restrict ourselves to the case $\mathbf{f}=0, g=0, \mathbf{b}=0, b=0$ and to consider the problem

$$
\begin{aligned}
& \varepsilon \mathbf{v}_{t}-\nabla^{2} \mathbf{v}+\ell(\mathbf{v})+\nabla p=0, \quad \nabla \cdot \mathbf{v}=0, \quad x \in \Omega, \quad t \in(0, T), \\
& \left.\mathbf{v}\right|_{t=0}=0, \\
& \left.\Pi_{0} S(\mathbf{v}) \mathbf{n}_{0}\right|_{\Gamma}=0, \\
& \mathbf{n}_{0} \cdot T(\mathbf{v}, p) \mathbf{n}_{0}-\mathbf{n}_{0} \cdot \Delta_{0} \int_{0}^{t} \mathbf{v} \mathrm{d} \tau=\int_{0}^{t} B \mathrm{~d} \tau,
\end{aligned}
$$

with $B \in \tilde{C}\left(0, T ; C^{\alpha}(\Gamma)\right)$.

For a fixed $\varepsilon>0$, the solvability of this problem in anisotropic Sobolev spaces was already established in [16], Theorem 5.1. Using similar scheme of the proof, we construct for arbitrary $B \in \tilde{C}\left(0, T ; C^{\alpha}(\Gamma)\right)$ a couple of functions $(\mathbf{u}, q)\left(\mathbf{u} \in \tilde{C}\left(0, T ; C^{2+\alpha}(\Omega)\right), \mathbf{u}_{t} \in \tilde{C}\left(0, T ; C^{\alpha}(\Omega)\right)\right)$ $q \in \tilde{C}\left(0, T ; C^{1+\alpha}(\Omega)\right)$, satisfying (3.7) with $B$ replaced by

$$
B_{1}=B+\mathcal{L} B,
$$

where $\mathcal{L}$ is a linear operator in the space $\tilde{C}\left(0, T ; C^{\alpha}(\Gamma)\right)$ such that $I+\mathcal{L}$ has a bounded inverse. This immediately implies the solvability of the problem (3.7).

For the construction of $\mathbf{u}, q$, we introduce on $\Gamma$ and in $\Omega_{\delta / 2}=\{x \in \Omega: \operatorname{dist}(x, \Gamma)<\delta / 2\}$ a smooth partition of unity $\left\{\zeta_{j}(x)\right\}_{j=1, \ldots, N}$ subordinated to the covering of $\Omega_{\delta / 2}$ with the balls $K_{j}=$ $\left\{\left|x-\xi^{j}\right|<\delta\right\}, \xi^{j} \in \Gamma$, and we assume that there exist smooth functions $\eta_{j}$, also with supp $\eta_{j} \subset K_{j}$, such that $\eta_{j} \zeta_{j}=\zeta_{j}$. Let $T_{j}$ be a tangent plane to $\Gamma$ at the point $\xi_{j}$ and let $\left(y_{1}^{j}, y_{2}^{j}, y_{3}^{j}\right)$ be a Cartesian coordinate system with the origin in $\xi^{j}$ and with $y_{3}^{j}$-axis directed along the interior normal $-\mathbf{n}_{0}\left(\xi^{j}\right)$. In the $d$-neighbourhood of the origin the surface $\Gamma$ can be given by the equation

$$
y_{3}^{j}=\phi^{j}\left(y_{1}^{j}, y_{2}^{j}\right), \quad \phi^{j} \in C^{3+\alpha}\left(\mathcal{B}_{d}\right),
$$

where $\mathcal{B}_{d}=\left\{\sqrt{y_{1}^{j 2}+y_{2}^{j 2}}<d\right\}$. Coordinates $\left(x_{1}, x_{2}, x_{3}\right)$ and $\left(y_{1}^{j}, y_{2}^{j}, y_{3}^{j}\right)$ are related to each other by the transformation

$$
y^{j}=U_{j}\left(x-\xi^{j}\right) \equiv U^{j}(x),
$$

where $U_{j}$ is an orthogonal matrix. The mapping

$$
x=Y^{j}(z)=\left(U^{j}\right)^{-1} \circ Z_{j} \circ U^{j}(z),
$$

where $Z_{j}\left(y^{j}\right) \equiv\left(y_{1}^{j}, y_{2}^{j}, y_{3}^{j}+\phi^{j}\left(y_{1}^{j}, y_{2}^{j}\right)\right)$ transforms the semi-ball $\left|z-\xi^{j}\right|<d,\left(\mathbf{z}-\boldsymbol{\xi}^{j}\right) \cdot \mathbf{n}\left(\xi^{j}\right)<0$, into a subdomain $\omega_{d} \subset \Omega$ containing $K_{j} \cap \Omega$. Since $\nabla \phi^{j}=0$ at the point $y_{1}^{j}=0, y_{2}^{j}=0$, the Jacobi matrix $J_{j}$ of the transformation (3.8) satisfies the condition

$$
J_{j}\left(\xi^{j}\right)=I .
$$


In the half-space $\mathbb{R}_{j}=\left\{\left(\mathbf{z}-\xi^{j}\right) \cdot \mathbf{n}\left(\xi^{j}\right)<0\right\}$ we consider the problem

$$
\begin{aligned}
& \varepsilon \mathbf{v}_{t}^{j}-\nabla^{2} \mathbf{v}^{j}+\nabla p^{j}=0, \quad \nabla \cdot \mathbf{v}^{j}=0, \quad z \in \mathbb{R}_{j}, \quad t \in(0, T), \\
& \left.\mathbf{v}^{j}\right|_{t=0}=0, \\
& \left.\Pi^{j} S\left(\mathbf{v}^{j}\right) \mathbf{n}_{0}\left(\xi^{j}\right)\right|_{T_{j}}=0, \\
& \mathbf{n}_{0}\left(\xi^{j}\right) \cdot T\left(\mathbf{v}^{j}, p^{j}\right) \mathbf{n}_{0}\left(\xi^{j}\right)-\left.\mathbf{n}_{0}\left(\xi^{j}\right) \cdot \Delta^{j} \int_{0}^{t} \mathbf{v}^{j} \mathrm{~d} \tau\right|_{T_{j}}=\int_{0}^{t} B^{j}(z, \tau) \mathrm{d} \tau,
\end{aligned}
$$

where $\Pi^{j}$ is a projector onto $T_{j}, \Delta^{j}$ is the Laplacean on $T_{j}$, and

$$
B^{j}(z, \tau)=\left.B(x, \tau) \zeta_{j}(x)\right|_{z=\left(Y^{j}\right)^{-1}(x)}, \quad x \in K_{j} \cap \Gamma .
$$

Further we set

$$
\begin{aligned}
\mathbf{u}^{j}(x, t)=\mathbf{v}^{j} \circ\left(Y^{j}\right)^{-1}, & q^{j}(x, t)=p^{j} \circ\left(Y^{j}\right)^{-1}, \\
\mathbf{u}^{\prime}(x, t)=\sum_{j} \eta_{j}(x) \mathbf{u}^{j}(x, t), & q^{\prime}(x, t)=\sum_{j} \eta_{j}(x) q^{j}(x, t),
\end{aligned}
$$

and we define $\left(\mathbf{u}^{\prime \prime}, q^{\prime \prime}\right)$ as the solution of the problem

$$
\begin{aligned}
& \varepsilon \mathbf{u}_{t}^{\prime \prime}-\nabla^{2} \mathbf{u}^{\prime \prime}+\ell\left(\mathbf{u}^{\prime \prime}\right)+\nabla q^{\prime \prime}=-\varepsilon \mathbf{u}_{t}^{\prime}+\nabla^{2} \mathbf{u}^{\prime}-\ell\left(\mathbf{u}^{\prime}\right)+\nabla q^{\prime} \equiv \mathbf{f}, \\
& \nabla \cdot \mathbf{u}^{\prime \prime}=-\nabla \cdot \mathbf{u}^{\prime} \equiv g, \quad x \in \Omega, \quad t \in(0, T) \\
& \left.\mathbf{u}^{\prime \prime}\right|_{t=0}=0 \\
& \left.\Pi_{0} S\left(\mathbf{u}^{\prime \prime}\right) \mathbf{n}_{0}\right|_{\Gamma}=-\left.\Pi_{0} S\left(\mathbf{u}^{\prime}\right) \mathbf{n}_{0}\right|_{\Gamma} \equiv \mathbf{b}, \\
& \left.\mathbf{n}_{0} \cdot T\left(\mathbf{u}^{\prime \prime}, q^{\prime \prime}\right) \mathbf{n}_{0}\right|_{\Gamma}=-\mathbf{n}_{0} \cdot T\left(\mathbf{u}^{\prime}, q^{\prime}\right) \mathbf{n}_{0}+\left.\sum_{j} \eta_{j}(x)\left(\mathbf{n}_{0}\left(\xi^{j}\right) \cdot T\left(\mathbf{v}^{j}, p^{j}\right) \mathbf{n}_{0}\left(\xi^{j}\right)\right)\right|_{z=Y_{j}^{-1}(x)} \equiv b .
\end{aligned}
$$

Then $\mathbf{u}=\mathbf{u}^{\prime}+\mathbf{u}^{\prime \prime}, q=q^{\prime}+q^{\prime \prime}$ is a solution of (3.7) with

$$
\left.B_{1}=B-\sum_{j}\left(\mathbf{n}_{0}(x) \cdot \Delta_{0}\left(\eta_{j} \mathbf{u}^{j}\right)-\mathbf{n}_{0}\left(\xi^{j}\right) \eta_{j} \cdot\left(\Delta^{j} \mathbf{v}^{j}\right) \circ\left(Y^{j}\right)^{-1}\right)\right)+\mathbf{n}_{0}(x) \cdot \Delta_{0} \mathbf{u}^{\prime \prime}
$$

instead of $B$. Hence,

$$
\mathcal{L} B=-\sum_{j}\left(\mathbf{n}_{0}(x) \cdot \Delta_{0}\left(\eta_{j} \mathbf{u}^{j}\right)-\mathbf{n}_{0}\left(\xi^{j}\right) \eta_{j} \cdot\left(\Delta^{j} \mathbf{v}^{j} \circ\left(Y^{j}\right)^{-1}\right)\right)+\mathbf{n}_{0}(x) \cdot \Delta_{0} \mathbf{u}^{\prime \prime} .
$$

Our main objective now is to prove that there holds the estimate

$$
\sup _{\tau<t}|\mathcal{L} B|_{C^{\alpha}(\Gamma)} \leqslant \varepsilon_{1}^{\prime} \sup _{\tau<t}|B(\cdot, \tau)|_{C^{\alpha}(\Gamma)}+c\left(\varepsilon_{1}^{\prime}, \varepsilon\right) \int_{0}^{t}|B(\cdot, \tau)|_{C^{\alpha}(\Gamma)} \mathrm{d} \tau
$$


with arbitrarily small $\varepsilon_{1}^{\prime}>0$. Then the existence of the bounded inverse operator $(I+\mathcal{L})^{-1}$ is evident.

At first we estimate $\mathbf{u}^{\prime \prime}, q^{\prime \prime}$. By virtue of (3.9), we have

$$
\begin{gathered}
\mathbf{f}(x, t)=-\ell\left(\mathbf{u}^{\prime}\right)+\sum_{j} q_{j} \nabla \eta_{j}+\sum_{j} \eta_{j}\left(\nabla-\nabla_{j}\right) q^{j}+2 \sum_{j} \nabla \mathbf{u}^{j} \nabla \eta_{j} \\
+\sum_{j} \mathbf{u}^{j} \nabla^{2} \eta_{j}+\sum_{j} \eta_{j}\left(\nabla^{2}-\nabla_{j}^{2}\right) \mathbf{u}^{j}, \\
g=-\sum_{j} \mathbf{u}^{j} \cdot \nabla \eta_{j}-\sum_{j} \eta_{j}\left(\nabla-\nabla_{j}\right) \cdot \mathbf{u}^{j}, \\
\mathbf{b}=-\sum_{j}\left(\Pi_{0} \nabla \eta_{j}\right)\left(\mathbf{u}^{j} \cdot \mathbf{n}\right)-\sum_{j} \frac{\partial \eta_{j}}{\partial n}\left(\Pi_{0} \mathbf{u}^{j}\right) \\
+\left.\sum_{j} \eta_{j}\left(\Pi^{j} S_{j}\left(\mathbf{u}^{j}\right) \mathbf{n}_{0}\left(\xi^{j}\right)-\Pi_{0} S\left(\mathbf{u}^{j}\right) \mathbf{n}_{0}(x)\right)\right|_{\Gamma} \\
b=-\left.2 \sum_{j} \frac{\partial \eta_{j}}{\partial n}\left(\mathbf{u}^{j} \cdot \mathbf{n}_{0}\right)\right|_{\Gamma}+\left.\sum_{j} \eta_{j}\left(\mathbf{n}_{0}\left(\xi^{j}\right) \cdot S_{j}\left(\mathbf{u}^{j}\right) \mathbf{n}_{0}\left(\xi^{j}\right)-\mathbf{n}_{0}(x) \cdot S\left(\mathbf{u}^{j}\right) \mathbf{n}_{0}(x)\right)\right|_{\Gamma}
\end{gathered}
$$

where $\nabla_{j}=J_{j}^{T} \nabla$ and

$$
S_{j}(\mathbf{u})=\left(\nabla_{j} \mathbf{u}\right)+\left(\nabla_{j} \mathbf{u}\right)^{T}=\sum_{m=1}^{2}\left(\left(J_{j}\right)_{m k} \frac{\partial u_{q}}{\partial x_{m}}+\left(J_{j}\right)_{m q} \frac{\partial u_{k}}{\partial x_{m}}\right)_{k, q=1,2,3 .}
$$

We observe also that, since det $J_{j}^{T}=1, g(x, t)$ can be written in the form

$$
\begin{gathered}
g=\nabla \cdot \mathbf{h}+h_{0}, \\
\mathbf{h}=\sum_{j} \eta_{j}\left(J_{j}-I\right) \mathbf{u}^{j}, \quad h_{0}=-\sum_{j} \nabla \eta_{j}\left(J_{j}-I\right) \mathbf{u}^{j}-\sum_{j} \mathbf{u}^{j} \cdot \nabla \eta_{j}=-\sum_{j} \nabla \eta_{j} \cdot J_{j} \mathbf{u}^{j} .
\end{gathered}
$$

Making use of the fact that $\left.\nabla_{j}\right|_{z=\xi^{j}}=\nabla$, of Theorem 6 and of interpolation inequalities (applied to $\mathbf{v}^{j}, p^{j}$ ), we obtain the estimate for $\left(\mathbf{u}^{\prime \prime}, q^{\prime \prime}\right)$

$$
\begin{gathered}
\varepsilon \sup _{\tau<t}\left|\mathbf{u}_{\tau}^{\prime \prime}(\cdot, \tau)\right|_{C^{\alpha}(\Omega)}+\sup _{\tau<t}\left|\mathbf{u}^{\prime \prime}(\cdot, \tau)\right|_{C^{2+\alpha}(\Omega)}+\sup _{\tau<t}\left|q^{\prime \prime}(\cdot, \tau)\right|_{C^{1+\alpha}(\Omega)} \leqslant \\
\leqslant c\left(\delta+\varepsilon_{1}\right) \max _{j}\left(\varepsilon \sup _{\tau<t}\left[\mathbf{v}_{\tau}^{j}(\cdot, \tau)\right]_{\mathbb{R}_{j}}^{(\alpha)}+\sup _{\tau<t}\left[\mathbf{v}^{j}(\cdot, \tau)\right]_{\mathbb{R}_{j}}^{(2+\alpha)}+\sup _{\tau<t}\left[p^{j}(\cdot, \tau)\right]_{\mathbb{R}_{j}}^{(1+\alpha)}\right) \\
+c\left(\delta, \varepsilon_{1}\right) \max _{j}\left(\sup _{\tau<t} \sup _{\mathbb{R}_{j}}\left|\mathbf{v}^{j}(z, \tau)\right|+\sup _{\tau<t} \sup _{\mathbb{R}_{j}}\left|p^{j}\left(z^{\prime}, \tau\right)\right|\right),
\end{gathered}
$$

and, since the principle part of $\Delta_{0}$ at the point $\xi^{j}$ coincides with $\Delta^{j}$, a similar inequality holds for $\mathcal{L} B$ :

$$
\sup _{\tau<t}|\mathcal{L} B|_{C^{\alpha}(\Gamma)} \leqslant c\left(\delta+\varepsilon_{1}\right) \max _{j}\left(\varepsilon \sup _{\tau<t}\left[\mathbf{v}_{\tau}^{j}(\cdot, \tau)\right]_{\mathbb{R}_{j}}^{(\alpha)}+\sup _{\tau<t}\left[\mathbf{v}^{j}(\cdot, \tau)\right]_{\mathbb{R}_{j}}^{(2+\alpha)}+\sup _{\tau<t}\left[p^{j}(\cdot, \tau)\right]_{\mathbb{R}_{j}}^{(1+\alpha)}\right)
$$




$$
+c\left(\delta, \varepsilon_{1}\right) \max _{j}\left(\sup _{\tau<t} \sup _{\mathbb{R}_{j}}\left|\mathbf{v}^{j}(z, \tau)\right|+\sup _{\tau<t} \sup _{\mathbb{R}_{j}}\left|p^{j}\left(z^{\prime}, \tau\right)\right|\right) .
$$

The first term in the right-hand side can be estimated by the inequality (2.31) for the problem (3.9). The function $p^{j}(z, t)$ is harmonic in $\mathbb{R}_{j}$ and satisfies the boundary conditions

$$
\left.p^{j}\right|_{T_{j}}=\left.\left(2 \mathbf{n}\left(\xi^{j}\right) \cdot \frac{\partial \mathbf{v}^{j}}{\partial n^{j}}-\mathbf{n}\left(\xi^{j}\right) \cdot \Delta^{j} \int_{0}^{t} \mathbf{v}^{j} \mathrm{~d} \tau\right)\right|_{T_{j}}-\int_{0}^{t} B^{j} \mathrm{~d} \tau^{\prime} \equiv p_{0}^{j}(z, t)
$$

hence,

$$
\begin{gathered}
\sup _{\mathbb{R}_{j}}\left|p^{j}(z, t)\right| \leqslant \sup _{T_{j}}\left|p_{0}^{j}(z, t)\right| \\
\leqslant \varepsilon_{2}\left[\mathbf{v}^{j}(\cdot, t)\right]_{\mathbb{R}_{j}}^{(2+\alpha)}+c\left(\varepsilon_{2}\right)\left[\mathbf{v}^{j}(\cdot, t)\right]_{\mathbb{R}_{j}}^{(\alpha)}+\int_{0}^{t}\left([\mathbf{v}(\cdot, \tau)]_{\mathbb{R}_{j}}^{(2+\alpha)}+[\mathbf{v}(\cdot, \tau)]_{\mathbb{R}_{j}}^{(\alpha)}\right) \mathrm{d} \tau+\int_{0}^{t} \sup _{T_{j}}\left|B^{j}(z, \tau)\right| \mathrm{d} \tau .
\end{gathered}
$$

Making use of the elementary estimate

$$
\left[\mathbf{v}^{j}(\cdot, t)\right]_{\mathbb{R}_{j}}^{(\alpha)} \leqslant \int_{0}^{t}\left[\mathbf{v}_{\tau}^{j}(\cdot, \tau)\right]_{\mathbb{R}_{j}}^{(\alpha)} \mathrm{d} \tau=\varepsilon^{-1} \int_{0}^{t} \varepsilon\left[\mathbf{v}_{\tau}^{j}(\cdot, \tau)\right]_{\mathbb{R}_{j}}^{(\alpha)} \mathrm{d} \tau
$$

and of (2.31), we obtain

$$
\begin{gathered}
\sup _{\mathbb{R}_{j}}\left|p^{j}(z, t)\right| \leqslant \varepsilon_{2}\left[\mathbf{v}^{j}(\cdot, t)\right]_{\mathbb{R}_{j}}^{(2+\alpha)}+c\left(\varepsilon_{2}, \varepsilon\right) \int_{0}^{t}\left(\left[B^{j}(\cdot, \tau)\right]_{T_{j}}^{(\alpha)}+\sup _{T_{j}}\left|B^{j}(z, \tau)\right|\right) \mathrm{d} \tau, \\
\int_{0}^{t} \sup _{\mathbb{R}_{j}}\left|p^{j}(z, \tau)\right| \mathrm{d} \tau \leqslant c\left(\varepsilon_{2}, \varepsilon\right) \int_{0}^{t}\left(\left[B^{j}(\cdot, \tau)\right]_{T_{j}}^{(\alpha)}+\sup _{T_{j}} \mid B^{j}(z, \tau \mid) \mathrm{d} \tau,\right.
\end{gathered}
$$

and, finally,

$$
\begin{gathered}
\sup _{\mathbb{R}_{j}}\left|\mathbf{v}^{j}(z, t)\right| \leqslant \varepsilon^{-1} \int_{0}^{t} \varepsilon \sup _{\mathbb{R}_{j}} \mid \mathbf{v}_{\tau}^{j}\left(z, \tau \mid \mathrm{d} \tau \leqslant \varepsilon^{-1} \int_{0}^{t}\left(\sup _{\mathbb{R}_{j}}\left|\nabla^{2} \mathbf{v}^{j}(z, \tau)\right|+\sup _{\mathbb{R}_{j}}\left|\nabla p^{j}(z, \tau)\right|\right) \mathrm{d} \tau\right. \\
\leqslant c \varepsilon^{-1} \int_{0}^{t}\left(\left[\mathbf{v}^{j}(\cdot, \tau)\right]_{\mathbb{R}_{j}}^{(2+\alpha)}+\left[\mathbf{v}^{j}(\cdot, \tau)\right]_{\mathbb{R}_{j}}^{(\alpha)}\right) \mathrm{d} \tau+c \varepsilon^{-1} \int_{0}^{t}\left(\left[\nabla p^{j}(\cdot, \tau)\right]_{\mathbb{R}_{j}}^{(\alpha)}+\sup _{\mathbb{R}_{j}}\left|p^{j}(z, \tau)\right|\right) \mathrm{d} \tau \\
\leqslant c(\varepsilon) \int_{0}^{t}\left(\left[B^{j}(\cdot, \tau)\right]_{T_{j}}^{(\alpha)}+\sup _{T_{j}}\left|B^{j}(z, \tau)\right|\right) \mathrm{d} \tau .
\end{gathered}
$$

If we introduce in $C^{\alpha}(\Gamma)$ the norm

$$
\|B\|_{C^{\alpha}(\Gamma)}=\max _{j}\left|B \zeta_{j}\right|_{C^{\alpha}\left(T_{j}\right)}
$$

and put all the above estimates together, we arrive at the inequality (3.10) for the norm $\|\mathcal{L} B\|_{C^{\alpha}(\Gamma)}$.

More careful arguments give for $\|\mathcal{L}\|$ a bound independent of $\varepsilon$.

The proof of Theorem 5 is now complete. 


\section{Proof of Theorem 2}

\subsection{Estimates of coefficients of operators $\nabla_{u}$ and $\Delta_{u}(t)$}

As mentioned in Section 1, the mapping $x=X_{u}(\xi, t)$ where $X_{u}$ is defined in (1.4) transforms the operator $\nabla=\left(\frac{\partial}{\partial x_{1}}, \frac{\partial}{\partial x_{2}}, \frac{\partial}{\partial x_{3}}\right)$ into $\nabla_{u}=\mathcal{A} \nabla=\left(\sum_{k=1}^{3} A_{k j} \frac{\partial}{\partial \xi_{k}}\right)_{j=1,2,3}$, if $\mathbf{u}$ satisfies the equation $\nabla_{u} \cdot \mathbf{u}=0$. The cofactor $A_{k j}$ of $a_{k j}=\delta_{k j}+\int_{0}^{t} \frac{\partial u_{k}}{\partial \xi_{j}} \mathrm{~d} \tau$ has the form

$$
A_{k j}=\delta_{k j}+B_{k j}
$$

where $B_{k j}$ is the sum of homogeneous linear and quadratic functions of $b_{i m}=\int_{0}^{t} \frac{\partial u_{i}}{\partial \xi_{m}} \mathrm{~d} \tau$. Sometimes, in order to avoid confusion, we denote $A_{k j}$ and $B_{k j}$ by $A_{k j}^{(u)}$ and $B_{k j}^{(u)}$, respectively.

There holds the following evident proposition.

Proposition 4.1 If $\mathbf{u}, \mathbf{w} \in \tilde{C}\left(0, T, C^{k+1+\alpha}(\Omega)\right), k=0,1, \ldots$, then

$$
\begin{aligned}
& \sup _{\tau<t}\left|A_{k j}^{(u)}(\cdot, \tau)-A_{k j}^{(w)}(\cdot, \tau)\right|_{C^{k+\alpha}(\Omega)} \\
& \quad \leqslant c \int_{0}^{t}|\mathbf{u}-\mathbf{w}|_{C^{k+1+\alpha}(\Omega)} \mathrm{d} \tau\left(1+\int_{0}^{t}|\mathbf{u}(\cdot, \tau)|_{C^{k+1+\alpha}(\Omega)} \mathrm{d} \tau+\int_{0}^{t}|\mathbf{w}(\cdot, \tau)|_{C^{k+1+\alpha}(\Omega)} \mathrm{d} \tau\right) .
\end{aligned}
$$

In particular,

$$
\sup _{\tau<t}\left|A_{k j}^{(u)}(\cdot, \tau)-\delta_{k j}\right|_{C^{k+\alpha}(\Omega)} \leqslant c \int_{0}^{t}|\mathbf{u}(\cdot, \tau)|_{C^{k+1+\alpha}(\Omega)} \mathrm{d} \tau\left(1+\int_{0}^{t}|\mathbf{u}(\cdot, \tau)|_{C^{k+1+\alpha}(\Omega)} \mathrm{d} t\right) .
$$

Moreover,

$$
\left|\frac{\partial}{\partial t} A_{k j}^{(u)}(\cdot, t)\right|_{C^{k+\alpha}(\Omega)} \leqslant c|\mathbf{u}(\cdot, t)|_{C^{k+1+\alpha}(\Omega)}\left(1+\int_{0}^{t}|\mathbf{u}(\cdot, \tau)|_{C^{k+1+\alpha}(\Omega)} \mathrm{d} \tau\right) .
$$

Let $\Gamma_{u}(t)=X_{u}(\Gamma)$ and let $\left(\eta_{1}, \eta_{2}\right)$ be local coordinates on the submanifold $\Gamma^{\prime} \subset \Gamma$. The normal $\mathbf{n}_{u}$ to $\Gamma_{u}^{\prime}(t)=X_{u}\left(\Gamma^{\prime}\right)$ and the Laplace-Beltrami operator $\Delta_{u}(t)$ on $\Gamma_{u}^{\prime}(t)$ are defined by

$$
\mathbf{n}_{u}= \pm \frac{\mathbf{r}_{\eta_{1}} \times \mathbf{r}_{\eta_{2}}}{\left|\mathbf{r}_{\eta_{1}} \times \mathbf{r}_{\eta_{2}}\right|}
$$

and

$$
\Delta_{u}(t) f=\frac{1}{\sqrt{g}} \sum_{\alpha, \beta=1}^{2} \frac{\partial}{\partial \eta_{\alpha}} \frac{\widehat{g}_{\alpha \beta}}{\sqrt{g}} \frac{\partial f}{\partial \eta_{\beta}} \equiv \sum_{\alpha, \beta=1}^{2} h_{\alpha \beta} \frac{\partial^{2} f}{\partial \xi_{\alpha} \partial \xi_{\beta}}+\sum_{\beta=1}^{2} h_{\beta} \frac{\partial f}{\partial \xi_{\beta}}
$$

where

$$
\begin{gathered}
\mathbf{r}\left(\eta_{1}, \eta_{2}, t\right)=\left.\mathbf{X}_{u}(\xi, t)\right|_{\xi=\xi\left(\eta_{1}, \eta_{2}\right)}=\boldsymbol{\xi}\left(\eta_{1}, \eta_{2}\right)+\int_{0}^{t} \omega\left(\eta_{1}, \eta_{2}, \tau\right) \mathrm{d} \tau, \\
\boldsymbol{\omega}\left(\eta_{1}, \eta_{2}, t\right)=\mathbf{u}\left(\xi\left(\eta_{1}, \eta_{2}\right), t\right),
\end{gathered}
$$


$g=\operatorname{det}\left(g_{\alpha \beta}\right)_{\alpha, \beta=1,2}, g_{\alpha \beta} \equiv g_{\alpha \beta}^{(u)}=\frac{\partial \mathbf{r}}{\partial \eta_{\alpha}} \cdot \frac{\partial \mathbf{r}}{\partial \eta_{\beta}}, \widehat{g}_{\alpha \beta}$ are elements of the associated matrix to $\left(g_{\alpha \beta}\right)_{\alpha, \beta=1,2}$, i.e.

$$
\begin{gathered}
\widehat{g}_{11}=g_{22}, \quad \widehat{g}_{22}=g_{11}, \quad \widehat{g}_{12}=\widehat{g}_{21}=-g_{12}, \\
h_{\alpha \beta}=\frac{\widehat{g}_{\alpha \beta}}{g}, \quad h_{\beta}=\frac{1}{\sqrt{g}} \sum_{\alpha=1}^{2} \frac{\partial}{\partial \xi_{\alpha}} \frac{\hat{g}^{\alpha \beta}}{\sqrt{g}} .
\end{gathered}
$$

We set $\dot{g}_{\alpha \beta}=\frac{\partial g_{\alpha \beta}}{\partial t}, \dot{h}_{\alpha \beta}=\frac{\partial h_{\alpha \beta}}{\partial t}, \dot{h}_{\beta}=\frac{\partial h_{\beta}}{\partial t}$ and

$$
\dot{\Delta}(t)=\sum_{\alpha, \beta=1}^{2} \dot{h}_{\alpha \beta} \frac{\partial^{2}}{\partial \xi_{\alpha} \partial \xi_{\beta}}+\sum_{\beta=1}^{2} \dot{h}_{\beta} \frac{\partial}{\partial \xi_{\beta}} .
$$

It follows from (4.2) that

$$
\begin{aligned}
& g_{\alpha \beta}=g_{\alpha \beta}^{(0)}+\frac{\partial \mathbf{r}_{0}}{\partial \eta_{\alpha}} \cdot \int_{0}^{t} \frac{\partial \boldsymbol{\omega}}{\partial \eta_{\beta}} \mathrm{d} \tau+\frac{\partial \mathbf{r}_{\alpha}}{\partial \eta_{\beta}} \cdot \int_{0}^{t} \frac{\partial \boldsymbol{\omega}}{\partial \eta_{\alpha}} \mathrm{d} \tau+\int_{0}^{t} \frac{\partial \boldsymbol{\omega}}{\partial \eta_{\alpha}} \mathrm{d} \tau \cdot \int_{0}^{t} \frac{\partial \boldsymbol{\omega}}{\partial \eta_{\beta}} \mathrm{d} \tau, \\
& \dot{g}_{\alpha \beta}=\frac{\partial \mathbf{r}_{0}(\eta)}{\partial \eta_{\alpha}} \cdot \frac{\partial \boldsymbol{\omega}(\eta, t)}{\partial \eta_{\beta}}+\frac{\partial \mathbf{r}_{0}}{\partial \eta_{\beta}} \cdot \frac{\partial \boldsymbol{\omega}}{\partial \eta_{\alpha}}+\frac{\partial \boldsymbol{\omega}}{\partial \eta_{\alpha}} \cdot \int_{0}^{t} \frac{\partial \boldsymbol{\omega}}{\partial \eta_{\beta}} \mathrm{d} \tau+\frac{\partial \boldsymbol{\omega}}{\partial \eta_{\beta}} \cdot \int_{0}^{t} \frac{\partial \boldsymbol{\omega}}{\partial \eta_{\alpha}} \mathrm{d} \tau, \\
& g_{\alpha \beta}^{(0)}=\frac{\partial \mathbf{r}_{0}}{\partial \eta_{\alpha}} \cdot \frac{\partial \mathbf{r}_{0}}{\partial \eta_{\beta}}, \quad \mathbf{r}_{0}=\boldsymbol{\xi}\left(\eta_{1}, \eta_{2}\right) .
\end{aligned}
$$

The following proposition is an easy consequence of formulas (4.1)-(4.4).

Proposition 4.2 Assume that

$$
\int_{0}^{t}|\mathbf{u}(\cdot, \tau)|_{C^{k+1+\alpha}(\Omega)} \mathrm{d} \tau+\int_{0}^{t}|\mathbf{w}(\cdot, \tau)|_{C^{k+1+\alpha}(\Omega)} \mathrm{d} \tau \leqslant \theta,
$$

where $\theta>0$ is so small that $\left|\mathbf{r}_{\eta_{1}} \times \mathbf{r}_{\eta_{2}}\right|$ and $g(\eta, t)$ are strictly positive. Then

$$
\begin{array}{r}
\left|\mathbf{n}_{u}(\cdot, t)-\mathbf{n}_{w}(\cdot, t)\right|_{C^{k+\alpha}\left(\Gamma^{\prime}\right)}+\left|g_{\alpha \beta}^{(u)}(\cdot, t)-g_{\alpha \beta}^{(w)}(\cdot, t)\right|_{C^{k+\alpha}\left(\Gamma^{\prime}\right)}+\left|h_{\alpha \beta}^{(u)}(\cdot, t)-h_{\alpha \beta}^{(w)}(\cdot, t)\right|_{C^{k+\alpha}\left(\Gamma^{\prime}\right)} \\
\leqslant c \int_{0}^{t}|\mathbf{u}-\mathbf{w}|_{C^{k+1+\alpha}\left(\Gamma^{\prime}\right)} \mathrm{d} \tau
\end{array}
$$

and if $k \geqslant 1$, then also

$$
\left|h_{\beta}^{(u)}-h_{\beta}^{(w)}\right|_{C^{k-1+\alpha}\left(\Gamma^{\prime}\right)} \leqslant c \int_{0}^{t}|\mathbf{u}(\xi(\cdot), \tau)-\mathbf{w}(\xi(\cdot), \tau)|_{C^{k+1+\alpha}\left(\Gamma^{\prime}\right)} \mathrm{d} \tau .
$$

Moreover,

$$
\begin{gathered}
\left|\dot{g}_{\alpha \beta}^{(u)}(\cdot, t)-\dot{g}_{\alpha \beta}^{(w)}(\cdot, t)\right|_{C^{k+\alpha}\left(\Gamma^{\prime}\right)}+\left|\dot{h}_{\alpha \beta}^{(u)}-\dot{h}_{\alpha \beta}^{(w)}\right|_{C^{k+\alpha}\left(\Gamma^{\prime}\right)} \leqslant c|\mathbf{u}(\xi(\cdot), t)-\mathbf{w}(\xi(\cdot), t)|_{C^{k+1+\alpha}\left(\Gamma^{\prime}\right)}, \\
\left|\dot{h}_{\beta}^{(u)}-\dot{h}_{\beta}^{(u)}\right|_{C^{k-1+\alpha}\left(\Gamma^{\prime}\right)} \leqslant c|\mathbf{u}(\xi(\cdot), t)-\mathbf{w}(\xi(\cdot), t)|_{C^{k+1+\alpha}\left(\Gamma^{\prime}\right)}, \quad k \geqslant 1, \\
\left|\frac{\partial}{\partial t} \mathbf{n}_{w}\right|_{C^{k+\alpha}\left(\Gamma^{\prime}\right)} \leqslant c|\mathbf{w}(\xi(\cdot), t)|_{C^{k+1+\alpha}\left(\Gamma^{\prime}\right)} \cdot
\end{gathered}
$$


COROllary 4.1 If $\mathbf{u}, \mathbf{w}$ satisfy the condition (4.5) with $k=1$, then

$$
\begin{gathered}
\begin{aligned}
\sup _{\tau<t}\left|\left(\nabla_{u}^{2}-\nabla_{w}^{2}\right) \mathbf{v}(\cdot, \tau)\right|_{C^{\alpha}(\Omega)} & +\sup _{\tau<t}\left|\Pi_{u} S_{u}(\mathbf{v}) \mathbf{n}_{u}-\Pi_{w} S_{w}(\mathbf{v}) \mathbf{n}_{w}\right|_{C^{1+\alpha}(\Gamma)} \\
& \quad+\sup _{\tau<t}\left|\mathbf{n}_{u} \cdot S_{u}(\mathbf{v}) \mathbf{n}_{u}-\mathbf{n}_{w} \cdot S_{w}(\mathbf{v}) \mathbf{n}_{w}\right|_{C^{1+\alpha}(\Gamma)}+\sup _{\tau<t}\left|\Delta_{u} \mathbf{v}-\Delta_{w} \mathbf{v}\right|_{C^{\alpha}(\Gamma)} e \\
& \leqslant c \sup _{\tau<t}|\mathbf{v}(\cdot, \tau)|_{C^{2+\alpha}(\Omega)} \int_{0}^{t}|\mathbf{u}(\cdot, \tau)-\mathbf{w}(\cdot, \tau)|_{C^{2+\alpha}(\Omega)} \mathrm{d} \tau
\end{aligned} \\
\sup _{\tau<t}\left|\left(\nabla_{u}-\nabla_{w}\right) p(\cdot, \tau)\right|_{C^{\alpha}(\Omega)} \leqslant c \sup _{\tau<t}|p(\cdot, \tau)|_{C^{1+\alpha}(\Omega)} \int_{0}^{t}|\mathbf{u}(\cdot, \tau)-\mathbf{w}(\cdot, \tau)|_{C^{1+\alpha}(\Omega)} \mathrm{d} \tau
\end{gathered}
$$

Proposition 4.3 There holds the estimate

$$
\begin{aligned}
\varepsilon^{\frac{1+\alpha}{2}} \sup _{\Gamma}[f \nabla u]_{(0, T)}^{\left(\frac{1+\alpha}{2}\right)} \leqslant & \left(\varepsilon \sup _{G_{T}}\left|f_{t}^{\prime}\right|+\underset{G_{T}}{\left.2 \sup _{T}|f|\right) \sup _{G_{T}}|\nabla u|}\right. \\
& +c \sup _{G_{T}}|f|\left(\varepsilon \sup _{t<T}\left|u_{t}(\cdot, t)\right|_{C^{\alpha}(\Omega)}+\sup _{G_{T}}|u(\cdot, t)|_{C^{2+\alpha}(\Omega)}\right)
\end{aligned}
$$

where $G_{T}=\Gamma \times(0, T)$.

Proof. Indeed, the left-hand side of (4.8) does not exceed

$$
\varepsilon^{\frac{1+\alpha}{2}} \sup _{\Gamma}[f]_{(0, T)}^{\left(\frac{1+\alpha}{2}\right)} \sup _{G_{T}}|\nabla u|+\sup _{G_{T}}|f| \varepsilon^{\frac{1+\alpha}{2}} \sup _{\Gamma}[\nabla u]_{(0, T)}^{\left(\frac{1+\alpha}{2}\right)} .
$$

Evaluating the Hölder constant of $\nabla u$ with respect to $t$ by (2.38) and taking into account that

$$
|f(\xi, t+h)-f(\xi, t)| \leqslant\left(\int_{t}^{t+h}\left|f_{\tau}^{\prime}(\xi, \tau)\right| \mathrm{d} \tau\right)^{\frac{1+\alpha}{2}}\left(2 \sup _{G_{T}}|f|\right)^{\frac{1-\alpha}{2}}
$$

and, as a consequence,

$$
\varepsilon^{\frac{1+\alpha}{2}} \sup _{\Gamma}[f]_{(0, T)}^{\left(\frac{1+\alpha}{2}\right)} \leqslant\left(\sup _{G_{T}}\left|f_{t}^{\prime}\right|\right)^{\frac{1+\alpha}{2}}\left(2 \sup _{G_{T}}|f|\right)^{\frac{1-\alpha}{2}},
$$

we easily obtain (4.8). The proposition is proved.

Inequality (4.8) may be used for the estimate of the Hölder constant of $\Pi_{u} S_{u}(\mathbf{v}) \mathbf{n}_{u}-$ $\Pi_{w} S_{w}(\mathbf{v}) \mathbf{n}_{w}$ with respect to $t$. We have:

$$
\begin{aligned}
& \varepsilon_{\Gamma}^{\frac{1+\alpha}{2}} \sup _{\Gamma}\left[\Pi_{u} S_{u}(\mathbf{v}) \mathbf{n}_{u}-\Pi_{w} S_{w}(\mathbf{v}) \mathbf{n}_{w}\right]_{(0, t)}^{\left(\frac{1+\alpha}{2}\right)} \\
& \leqslant c\left(\int_{0}^{t} \sup _{\Gamma}|\nabla(\mathbf{u}(\xi, \tau)-\mathbf{w}(\xi, \tau))| \mathrm{d} \tau+\varepsilon \sup _{\tau<t} \sup _{G_{t}}|\nabla(\mathbf{u}(\xi, \tau)-\mathbf{w}(\xi, \tau))|\right) \\
& \times\left(\varepsilon \sup _{\tau<t}\left|\mathbf{v}_{\tau}(\cdot, \tau)\right|_{C^{\alpha}(\Omega)}+\sup _{\tau<t}|\mathbf{v}(\cdot, \tau)|_{C^{2+\alpha}(\Omega)}\right) .
\end{aligned}
$$




\subsection{Quasistationary approximation}

Assume that $\Gamma \in C^{4+\alpha}$. Then, according to Theorem 1, there exists a unique solution of the problem (1.8) defined on a certain finite time interval $\left(0, T_{0}\right)$ and satisfying conditions (1.9) and inequality (1.10) for $\ell=1+\alpha$. It follows that

$$
\begin{aligned}
\int_{0}^{T}|\mathbf{w}(\cdot, t)|_{C^{3+\alpha}(\Omega)} \mathrm{d} t & \leqslant T \sup _{t \in(0, T)}|\mathbf{w}(\cdot, t)|_{C^{3+\alpha}(\Omega)} \\
& \leqslant c T\left(\left|H_{0}\right|_{C^{2+\alpha}(\Gamma)}+\sum_{k=1}^{6}\left|\mu_{k}\right|\right), \quad T \leqslant T_{0} .
\end{aligned}
$$

Let $\tau \in\left(0, T_{0}\right), t \in\left(\tau, T_{0}\right)$ and

$$
\mathbf{w}^{(\tau)}(x, t)=\mathbf{w}\left(X_{w}^{-1}(x, \tau), t\right), \quad x \in \Omega_{\tau} .
$$

Clearly,

$$
\frac{\partial \mathbf{w}^{(\tau)}}{\partial x_{k}}=\left.\sum_{j=1}^{3} A_{j k}(\xi, \tau) \frac{\partial \mathbf{w}(\xi, t)}{\partial \xi_{j}}\right|_{\xi=X_{w}^{-1}(x, \tau)} .
$$

Differentiating this formula we can easily show that $\mathbf{w}^{(\tau)} \in C^{3+\alpha}\left(\Omega_{T}\right)$ and that

$$
\sup _{t \in\left(\tau, T_{0}\right)}\left|\mathbf{w}^{(\tau)}(\cdot, t)\right|_{C^{3+\alpha}\left(\Omega_{T}\right)} \leqslant c,
$$

where $c$ depends on $T_{0}$ and on $\left|H_{0}\right|_{C^{2+\alpha}(\Gamma)}+\sum_{k=1}^{6}\left|\mu_{k}\right|$.

We need also the estimate of the time derivative $\mathbf{w}_{t}(\xi, t)$.

Proposition 4.4 If $\Gamma \in C^{4+\alpha}$, then $\mathbf{w}_{t} \in C^{1+\alpha}(\Omega)$ and

$$
\left|\mathbf{w}_{t}(\cdot, t)\right|_{C^{\alpha}(\Omega)} \leqslant c_{1},
$$

where $c_{1}$ depends on the sum of norms $\sup _{\tau<t}|\mathbf{w}(\cdot, \tau)|_{C^{3+\alpha}(\Omega)}+|r(\cdot, t)|_{C^{1+\alpha}(\Omega)}$.

Proof. Differentiation of (1.8) and (1.9) with respect to $t$ gives

$$
\begin{aligned}
& -\nabla_{w}^{2} \mathbf{w}_{t}+\nabla_{w} r_{t}=\left(\nabla_{w} \cdot \dot{\nabla}_{w}+\dot{\nabla}_{w} \cdot \nabla_{w}\right) \mathbf{w}-\dot{\nabla}_{w} r \equiv \mathbf{f}(\xi, t), \\
& \nabla_{w} \cdot \mathbf{w}_{t}=-\dot{\nabla}_{w} \cdot \mathbf{w} \equiv g(\xi, t), \\
& \left.T_{w}\left(\mathbf{w}_{t}, r_{t}\right) \mathbf{n}_{w}\right|_{\Gamma}=-\dot{S}_{w}(\mathbf{w}, r) \mathbf{n}_{w}-T_{w}(\mathbf{w}, r) \dot{\mathbf{n}}_{w}+\left(\Delta_{w}(t) \mathbf{w}+\dot{\Delta}_{w}(t) \mathbf{X}_{w}\right) \equiv \mathbf{a}(\xi, t), \\
& \int_{\Omega} \mathbf{w}_{t}(\xi, t) \cdot \boldsymbol{\varphi}_{k}\left(X_{w}(\xi, t)\right) \mathrm{d} \xi=-\int_{\Omega} \mathbf{w}(\xi, t) \cdot \frac{\partial}{\partial t} \boldsymbol{\varphi}_{k}\left(X_{w}(\xi, t)\right) \mathrm{d} \xi \equiv v_{k},
\end{aligned}
$$


where

$$
\begin{aligned}
& \dot{\nabla}_{w}=\left(\sum_{k=1}^{3} \frac{\partial A_{k j}}{\partial t} \frac{\partial}{\partial \xi_{j}}\right)_{j=1,2,3}, \\
& \dot{\mathbf{n}}_{w}=\frac{\partial}{\partial t} \mathbf{n}_{w}(\xi, t), \\
& \dot{S}_{w}(\mathbf{v})=\left(\sum_{k=1}^{3}\left(\frac{\partial A_{k j}}{\partial t} \frac{\partial v_{i}}{\partial \xi_{j}}+\frac{\partial A_{i j}}{\partial t} \frac{\partial v_{k}}{\partial \xi_{j}}\right)\right)_{k, i=1,2,3} .
\end{aligned}
$$

Clearly, $\mathbf{f} \in C^{1+\alpha}(\Omega), g \in C^{2+\alpha}(\Omega), \mathbf{a} \in C^{1+\alpha}(\Gamma)$. In the Eulerian coordinates, (4.12) takes the form

$$
\begin{aligned}
& -\nabla^{2} \mathbf{w}^{\prime}+\nabla r^{\prime}=\mathbf{f}^{\prime}, \quad \nabla \cdot \mathbf{w}^{\prime}=g^{\prime}, \quad \text { in } \Omega_{w}(t)=X_{w}(\Omega), \\
& T\left(\mathbf{w}^{\prime}, r^{\prime}\right) \mathbf{n}=\mathbf{a}^{\prime} \quad \text { on } \Gamma_{w}(t)=\partial \Omega_{w}(t), \\
& \int_{\Omega_{w}} \mathbf{w}^{\prime} \cdot \varphi_{k}(x) \mathrm{d} x=v_{k}, \quad k=1, \ldots, 6,
\end{aligned}
$$

where

$$
f^{\prime}(x, t)=f\left(X_{w}^{-1}(x, t), t\right), \quad x \in \Omega_{w}(t) .
$$

Inequality (4.11) follows from (4.10) and from the Schauder estimate for the problem (4.13). The proposition is proved.

\subsection{Solvability of the problem (1.11)}

We proceed to the proof of Theorem 2 and consider at first a more general problem

$$
\begin{aligned}
& \varepsilon \mathbf{V}_{t}-\nabla_{w}^{2} \mathbf{V}+\ell_{w}(\mathbf{V})+\nabla_{w} P=\mathbf{f}, \\
& \nabla_{w} \cdot \mathbf{V}=g, \\
& \left.\mathbf{V}\right|_{t=0}=\mathbf{V}_{0}(\xi), \quad \xi \in \Omega, \\
& \left.T_{w}(\mathbf{V}, P) \mathbf{n}_{w}\right|_{\Gamma}=\mathbf{a},
\end{aligned}
$$

on a certain small time interval $\left(0, t_{1}\right)$.

Proposition 4.5 Assume that $\Gamma \in C^{3+\alpha}, \alpha \in(0,1), \mathbf{f} \in \tilde{C}\left(0, T ; C^{\alpha}(\Omega)\right), g \in$ $\tilde{C}\left(0, T ; C^{1+\alpha}(\Omega)\right), \mathbf{v}_{0} \in C^{2+\alpha}(\Omega), \mathbf{a} \in \tilde{C}\left(0, T ; C^{1+\alpha}(\Gamma)\right), \Pi_{w} \mathbf{a} \in C^{1+\alpha,(1+\alpha) / 2}(\Gamma \times(0, T))$, and that there are satisfied the conditions

$$
\nabla \cdot \mathbf{V}_{0}(\xi)=g(\xi, 0),\left.\quad \Pi_{0} \mathbf{V}_{0}(\xi)\right|_{\Gamma}=\Pi_{0} \mathbf{a}(\xi, 0)
$$

and

$$
g_{t}=\nabla \cdot \mathbf{h}(\xi, t)+h_{0}(\xi, t),
$$

where $\mathbf{h}, h_{0}$ have the time derivatives $\mathbf{h}_{t} \in \tilde{C}\left(0, T, C^{\alpha}(\Omega)\right), h_{0 t} \in C(0, T, C(\Omega))$. There exists such $t_{1}>0$ depending on $\mathbf{w}$ (see condition (4.17)) that problem (4.14) has a unique solution 
$\mathbf{V} \in \tilde{C}\left(0, t_{1}, C^{\alpha}(\Omega)\right), P \in \tilde{C}\left(0, t_{1}, C^{1+\alpha}(\Omega)\right)$ such that $\mathbf{V}_{t} \in \tilde{C}\left(0, t_{1}, C^{\alpha}(\Omega)\right)$, and it satisfies the inequality

$$
Y_{t}(\mathbf{V}, P) \leqslant c N(t), \quad t \in\left(0, t_{1}\right)
$$

where

$$
\begin{aligned}
Y_{t}(\mathbf{V}, P) & =\varepsilon \sup _{\tau<t}\left|\mathbf{V}_{\tau}(\cdot, \tau)\right|_{C^{\alpha}(\Omega)}+\sup _{\tau<t}|\mathbf{V}(\cdot, \tau)|_{C^{2+\alpha}(\Omega)}+\sup _{\tau<t}|P(\cdot, \tau)|_{C^{\alpha}(\Omega)}, \\
N(t)= & \sup _{\tau<t}|\mathbf{f}(\cdot, \tau)|_{C^{\alpha}(\Omega)}+\sup _{\tau<t}|g(\cdot, \tau)|_{C^{1+\alpha}(\Omega)}+\sup _{\tau<t}\left|\mathbf{h}_{\tau}(\cdot, \tau)\right|_{C^{\alpha}(\Omega)} \\
& +\sup _{\tau<t} \sup _{\Omega}\left|h_{0 \tau}(\xi, t)\right|+\sup _{\tau<t}|\mathbf{a}(\cdot, \tau)|_{C^{1+\alpha}(\Gamma)}+\varepsilon^{\frac{1+\alpha}{2}} \sup _{\Gamma}\left[\Pi_{w} \mathbf{a}\right]_{(0, t)}^{\left(\frac{1+\alpha}{2}\right)} .
\end{aligned}
$$

Proof. If $\mathbf{n}_{w} \cdot \mathbf{n}_{0}>0$ (which is the case for small $t$ ), then we can write (4.14) in the form

$$
\begin{aligned}
& \varepsilon \mathbf{V}_{t}-\nabla^{2} \mathbf{V}+\ell(\mathbf{V})+\nabla P=M_{1}(\mathbf{V}, P)+\mathbf{f}, \\
& \nabla \cdot \mathbf{V}=M_{2}(\mathbf{V})+g, \\
& \left.\mathbf{V}\right|_{t=0}=\mathbf{V}_{0}, \\
& \left.\Pi_{0} S(\mathbf{V}) \mathbf{n}_{0}\right|_{\Gamma}=M_{3}(\mathbf{V})+\Pi_{0} \Pi_{w} \mathbf{a}, \\
& \left.\mathbf{n}_{0} \cdot T(\mathbf{V}, P) \mathbf{n}_{0}\right|_{\Gamma}=M_{4}(\mathbf{V})+\mathbf{a} \cdot \mathbf{n}_{w},
\end{aligned}
$$

where

$$
\begin{aligned}
M_{1}(\mathbf{V}, P)= & \left(\nabla_{w}^{2}-\nabla^{2}\right) \mathbf{V}+\left(\nabla-\nabla_{w}\right) P+\sum_{k=1}^{6} \int_{\Omega}\left[\mathbf{V}\left(\xi^{\prime}, t\right) \cdot \boldsymbol{\varphi}_{k}\left(\xi^{\prime}\right) \boldsymbol{\varphi}_{k}(\xi)\right. \\
& \left.-\mathbf{V}\left(\xi^{\prime}, t\right) \cdot \boldsymbol{\varphi}_{k}\left(X_{w}\left(\xi^{\prime}, t\right)\right) \boldsymbol{\varphi}_{k}\left(X_{w}(\xi, t)\right)\right] d \xi^{\prime}, \\
M_{2}(\mathbf{V})= & \left(\nabla-\nabla_{w}\right) \cdot \mathbf{V}=\nabla H, \quad H=\left(I-\mathcal{A}^{(w)}\right)^{T} \mathbf{V}, \\
M_{3}(\mathbf{V})= & \Pi_{0}\left(\Pi_{0} S(\mathbf{V}) \mathbf{n}_{0}-\Pi_{w} S_{w}(\mathbf{V}) \mathbf{n}_{w}\right), \\
M_{4}(\mathbf{V})= & \mathbf{n}_{0} \cdot S(\mathbf{V}) \mathbf{n}_{0}-\mathbf{n}_{w} \cdot S_{w}(\mathbf{V}) \mathbf{n}_{w} .
\end{aligned}
$$

and $\mathcal{A}^{T}$ means a transposed matrix. In virtue of (4.6), (4.7), and (4.9),

$$
\begin{aligned}
\sup _{\tau<t}\left|M_{1}(\mathbf{V}, P)\right|_{C^{\alpha}(\Omega)}+\sup _{\tau<t}\left|M_{2}(\mathbf{V})\right|_{C^{1+\alpha}(\Omega)}+ & \sup _{\tau<t}\left|M_{3}(\mathbf{V})\right|_{C^{1+\alpha}(\Omega)}+\sup _{\tau<t}\left|M_{4}(\mathbf{V})\right|_{C^{1+\alpha}(\Gamma)} \\
& +\varepsilon_{\Gamma}^{\frac{1+\alpha}{2}} \sup _{\Gamma}\left[\Pi_{0} M_{3}(\mathbf{V})\right]_{(0, t)}^{\left(\frac{1+\alpha}{2}\right)}+\varepsilon \sup \left|H_{t}(\mathbf{V})\right|_{C^{\alpha}(\Omega)} \\
\leqslant c\left(\int_{0}^{t}|\mathbf{w}(\cdot, \tau)|_{C^{2+\alpha}(\Omega)} \mathrm{d} \tau\right. & \left.+\varepsilon \sup _{\tau<t}|\mathbf{w}(\cdot, \tau)|_{C^{2+\alpha}(\Omega)}\right) Y_{t}(\mathbf{V}, P) .
\end{aligned}
$$


Therefore, if $t_{1}$ is so small that

$$
c c^{\prime}\left(\int_{0}^{t_{1}}|\mathbf{w}(\cdot, \tau)|_{C^{2+\alpha}(\Omega)} \mathrm{d} \tau+\varepsilon \sup _{\tau<t_{1}}|\mathbf{w}(\cdot, \tau)|_{C^{2+\alpha}(\Omega)}\right) \leqslant \frac{1}{2},
$$

where $c^{\prime}$ is the constant in the inequality (1.25), then the solvability of the problem (4.14) and the estimate (4.15) follow from Theorem 6 and from the contraction mapping principle. The proposition is proved.

We have constructed the solution for $t \in\left(0, t_{1}\right)$. To extend it to a larger time interval, we consider the same problem for $t>t_{1}$, taking $\mathbf{V}\left(\xi, t_{1}\right)$ as an initial function and introducing a new independent variable

$$
\xi^{\prime}=X_{w}\left(\xi, t_{1}\right) \in \Omega^{\prime} \equiv X_{w}\left(\Omega, t_{1}\right) .
$$

Then this problem takes the form

$$
\begin{aligned}
& \varepsilon \mathbf{V}_{t}^{\prime}-\nabla_{w}^{\prime 2} \mathbf{V}^{\prime}+\ell_{w}^{\prime}\left(\mathbf{V}^{\prime}\right)+\nabla_{w}^{\prime} P^{\prime}=\mathbf{f}^{\prime}, \\
& \nabla_{w}^{\prime} \cdot \mathbf{V}^{\prime}=g^{\prime} \quad\left(\xi^{\prime} \in \Omega^{\prime}, t>t_{1}\right), \\
& \left.\mathbf{V}^{\prime}\right|_{t=t_{1}}=\mathbf{V}_{1}\left(\xi^{\prime}\right) \equiv \mathbf{V}\left(X_{w}^{-1}\left(\xi^{\prime}, t_{1}\right)\right), \\
& \left.T_{w}^{\prime}\left(\mathbf{V}^{\prime}, P^{\prime}\right) \mathbf{n}_{w}^{\prime}\right|_{\Gamma^{\prime}}=\mathbf{a}^{\prime},
\end{aligned}
$$

where $F^{\prime}\left(\xi^{\prime}, t\right)=F\left(X_{w}^{-1}\left(\xi^{\prime}, t_{1}\right), t\right)\left(t \geqslant t_{1}\right), \Gamma^{\prime}=\partial \Omega^{\prime}, \mathbf{n}_{w}^{\prime}$ is the exterior normal to the surface $\Gamma_{w}(t)$ which is now considered as $X_{w}^{\prime}\left(\Gamma^{\prime}, t\right)$, and

$$
\mathbf{X}_{w}^{\prime}\left(\xi^{\prime}, t\right)=\xi^{\prime}+\int_{t_{1}}^{t} \mathbf{w}^{\prime}\left(\xi^{\prime}, \tau\right) \mathrm{d} \tau, \quad \mathbf{w}^{\prime}\left(\xi^{\prime}, \tau\right)=\mathbf{w}\left(X_{w}^{-1}\left(\xi^{\prime}, t_{1}\right), \tau\right) .
$$

The operators $\nabla_{w}^{\prime}, T_{w}^{\prime}, \ell_{w}^{\prime}$ are expressed in terms of the transformation $X_{w}^{\prime}$ in the same way as $\nabla_{w}, T_{w}, \ell_{w}$ are expressed in terms of $X_{w}$. Finally, it is easy to see that

$$
g^{\prime}=\nabla \cdot \mathbf{h}^{\prime \prime}+h_{0}^{\prime}
$$

where

$$
\mathbf{h}_{j}^{\prime \prime}=\left.\sum_{j=1}^{3}\left(\delta_{j k}+\int_{0}^{t_{1}} \frac{\partial w_{j}^{\prime}(\xi, \tau)}{\partial \xi_{k}} \mathrm{~d} \tau\right)\right|_{\xi=X_{w}^{-1}\left(\xi^{\prime}, t_{1}\right)} h_{k}^{\prime}\left(\xi^{\prime}, t\right) .
$$

Due to uniform boundedness of the norm of $\mathbf{w}^{(\tau)}(x, t)$ (see (4.10)), problem (4.18) can be solved by the same method on the time interval $t \in\left(t_{1}, 2 t_{1}\right)$, and the solution can be estimated by the norms of the data $\mathbf{f}^{\prime}, g^{\prime}, \mathbf{h}^{\prime \prime}, h_{0}^{\prime}, \mathbf{V}_{1}, \mathbf{a}^{\prime}$ exactly as above (see (4.15)). This proves the solvability of Problem (4.14) and the estimate (4.15) for $t \in\left(0,2 t_{1}\right)$. Repeating this argument we can extend the solution into the whole interval $\left(0, T_{0}\right)$ and prove inequality (4.15) for $t \in\left(0, T_{0}\right)$.

(1.13) is just a particular case of (4.15). 
4.4 Proof of estimates (1.14)-(1.16)

Problem (1.11) can be written in the Eulerian coordinates $x=X_{w}(\xi, t)$ in the form

$$
\begin{aligned}
& \varepsilon\left(\mathbf{v}_{t}+(\mathbf{w} \cdot \nabla) \mathbf{v}\right)-\nabla^{2} \mathbf{v}+\ell(\mathbf{v})+\nabla p=0, \quad \nabla \cdot \mathbf{v}=0, \quad x \in \Omega_{w}(t), \\
& \left.\mathbf{v}\right|_{t=0}=\mathbf{V}_{0}(x), \quad x \in \Omega_{0}, \\
& \left.T(\mathbf{v}, p) \mathbf{n}\right|_{\Gamma_{w}(t)}=0 .
\end{aligned}
$$

In order to prove (1.14), we multiply the first equation by $\mathbf{v}$ and integrate over $\Omega_{w}(t)$ which gives

$$
\frac{\varepsilon}{2} \frac{\mathrm{d}}{\mathrm{d} t}\|\mathbf{v}(\cdot, t)\|_{L_{2}\left(\Omega_{w}(t)\right)}^{2}+\frac{1}{2}\|S(\mathbf{v})\|_{L_{2}\left(\Omega_{w}(t)\right)}^{2}+\sum_{k=1}^{6}\left(\int_{\Omega_{w}(t)} \mathbf{v} \cdot \boldsymbol{\varphi}_{k} \mathrm{~d} x\right)^{2}=0 .
$$

By the Korn inequality,

$$
\frac{1}{2}\|S(\mathbf{v})\|_{L^{2}\left(\Omega_{w}(t)\right)}^{2}+\sum_{k=1}^{6}\left(\int_{\Omega_{w}(t)} \mathbf{v} \cdot \boldsymbol{\varphi}_{k} \mathrm{~d} x\right)^{2} \geqslant c \mid \mathbf{v} \|_{L_{2}\left(\Omega_{w}(t)\right)}^{2}
$$

with a certain positive constant $c$ independent of $t$. Hence, (4.19) yields

$$
\|\mathbf{v}(\cdot, t)\|_{L_{2}\left(\Omega_{w}(t)\right)}^{2} \leqslant e^{-\frac{2 c}{\varepsilon}}\left\|\mathbf{V}_{0}\right\|_{L_{2}(\Omega)}^{2}
$$

which is equivalent to (1.14).

The proof of (1.15) and (1.16) is based on (1.14) and on the following two propositions.

Proposition 4.6 If $\Gamma \in C^{3+\alpha}$, then the solution of Problem (1.11) satisfies the inequality

$$
\begin{array}{r}
\varepsilon \sup _{t-\delta<\tau<t}\left|\mathbf{V}_{\tau}(\cdot, \tau)\right|_{C^{\alpha}(\Omega)}+\sup _{t-\delta<\tau<t}|\mathbf{V}(\cdot, \tau)|_{C^{2+\alpha}(\Omega)}+\sup _{\tau-\delta<\tau<t}|\mathbf{P}(\cdot, \tau)|_{C^{1+\alpha}(\Omega)} \\
\leqslant c \varepsilon \delta^{-1}\left(1+\varepsilon \delta^{-1}\right)^{\frac{2 \alpha+3}{4}} \sup _{t-2 \delta<\tau<t}\|\mathbf{V}(\cdot, \tau)\|_{L_{2}(\Omega),}
\end{array}
$$

where $t \in\left(\varepsilon, T_{0}\right), \delta=\min \left(t / 4, t_{1} / 4\right), t_{1}$ is the same as in Proposition 4.5.

Proof. We fix $t_{0} \in\left(0, T_{0}\right), \delta_{0}=\min \left(\frac{t_{0}}{4}, \frac{t_{1}}{4}\right)$ and introduce the function $\omega_{\lambda}(t), t>0$, which is smooth, monotone, equals zero for $t<t_{0}-2 \delta_{0}+\frac{\lambda}{2}$, equals one for $t>t_{0}-2 \delta_{0}+\lambda\left(\lambda \in\left(0, \delta_{0}\right)\right)$ and satisfies the estimate

$$
\left|\omega_{\lambda}^{\prime}(t)\right| \leqslant c \lambda^{-1}
$$

The functions $\mathbf{U}=\mathbf{V} \omega_{\lambda}, Q=P \omega_{\lambda}$ satisfy the equations

$$
\begin{aligned}
& \varepsilon \mathbf{U}_{t}+\ell_{w}(\mathbf{U})-\nabla_{w}^{2} \mathbf{U}+\nabla_{w} Q=\varepsilon \mathbf{V} \omega_{\lambda}^{\prime}(t), \\
& \nabla_{w} \cdot \mathbf{U}=0, \\
& \left.\mathbf{U}\right|_{t=0}=0, \\
& \left.T_{w}(\mathbf{U}, Q) \mathbf{n}_{w}\right|_{\Gamma}=0,
\end{aligned}
$$


hence, by virtue of (4.15),

$$
Y_{t_{0}}(\mathbf{U}, Q) \leqslant c_{0} \varepsilon \lambda^{-1} \sup _{t-2 \delta_{0}+\lambda / 2<\tau<t}|\mathbf{V}(\cdot, \tau)|_{C^{\alpha}(\Omega)} .
$$

We estimate the right-hand side by the interpolation inequality

$$
|\mathbf{V}(\cdot, \tau)|_{C^{\alpha}(\Omega)} \leqslant \mu|\mathbf{V}(\cdot, \tau)|_{C^{2+\alpha}(\Omega)}+c \mu^{-\frac{2 \alpha+3}{4}}\|\mathbf{V}(\cdot, \tau)\|_{L_{2}(\Omega)}
$$

which is true for arbitrary $\mu<1$, set

$$
\mu=\frac{\chi}{1+\varepsilon \lambda^{-1}}, \quad \chi \in(0,1),
$$

and multiply (4.21) by $\lambda\left(1+\varepsilon \lambda^{-1}\right)^{-(3 / 4+\alpha / 2)}$. This gives

$$
f(\lambda) \leqslant 2 c_{0} \chi \frac{\varepsilon \lambda^{-1}}{1+\varepsilon \lambda^{-1}}\left(\frac{1+2 \varepsilon \lambda^{-1}}{1+\varepsilon \lambda^{-1}}\right)^{\frac{3}{4}+\frac{\alpha}{2}} f\left(\frac{\lambda}{2}\right)+c \varepsilon \chi^{-\frac{\alpha}{2}-\frac{3}{4}} \sup _{t_{0}-2 \delta_{0}<t<t_{0}}\|\mathbf{V}(\cdot, \tau)\|_{L_{2}(\Omega)}
$$

where

$$
\begin{aligned}
f(\lambda)= & \lambda\left(1+\varepsilon \lambda^{-1}\right)^{-\left(\frac{3}{4}+\frac{\alpha}{2}\right)}\left(\varepsilon \sup _{t_{0}-2 \delta_{0}+\lambda<\varepsilon<t_{0}}\left|\mathbf{V}_{\tau}(\cdot, \tau)\right|_{C^{\alpha}(\Omega)}\right. \\
& \left.+\sup _{t_{0}-2 \delta_{0}+\lambda<\varepsilon<t_{0}}|\mathbf{V}(\cdot, \tau)|_{C^{2+\alpha}(\Omega)}+\sup _{t_{0}-2 \delta_{0}+\lambda<\varepsilon<t_{0}}|P(\cdot, \tau)|_{C^{1+\alpha}(\Omega)}\right) .
\end{aligned}
$$

If

$$
2^{\frac{11}{4}+\frac{\alpha}{2}} c_{0} \chi<1
$$

then the last inequality implies

$$
f(\lambda) \leqslant \frac{1}{2} f\left(\frac{\lambda}{2}\right)+\varepsilon c_{0} \chi^{-\frac{\alpha}{2}-\frac{3}{4}} \sup _{t_{0}-2 \delta_{0}<\tau<t_{0}}\|\mathbf{V}(\cdot, \tau)\|_{L_{2}(\Omega)},
$$

and, since $f(0)=0$,

$$
f(\lambda) \leqslant 2 c_{0} \varepsilon \chi^{-\frac{\alpha}{2}-\frac{3}{4}} \sup _{t_{0}-2 \delta_{0}<\tau<t_{0}}\|\mathbf{V}(\cdot, \tau)\|_{L_{2}(\Omega)},
$$

or

$$
\begin{gathered}
\varepsilon \sup _{t_{0}-2 \delta_{0}+\lambda<\tau<t_{0}}\left|\mathbf{V}_{t}(\cdot, \tau)\right|_{C^{\alpha}(\Omega)}+\sup _{t_{0}-2 \delta_{0}+\lambda<\tau<t_{0}}|\mathbf{V}(\cdot, \tau)|_{C^{2+\alpha}(\Omega)}+ \\
+\sup _{t_{0}-2 \delta_{0}+\lambda<\tau<t_{0}}|P(\cdot, \tau)|_{C^{1+\alpha}(\Omega)} \\
\leqslant 2 c_{0} \varepsilon \lambda^{-1}\left(1+\varepsilon \lambda^{-1}\right)^{\frac{2 \alpha+3}{4}} \sup _{t_{0}-2 \delta_{0}<\tau<t_{0}}\|\mathbf{V}(\cdot, \tau)\|_{L_{2}(\Omega)} .
\end{gathered}
$$

Setting here $\lambda=\delta_{0}$, we arrive at (4.20) for arbitrary $t_{0}<T_{0}$. The proposition is proved. 
Proposition 4.7 If $\Gamma \in C^{4+\alpha}, \mathbf{V}_{0} \in C^{3+\alpha}(\Omega)$ and conditions

$$
\nabla \cdot \mathbf{V}_{0}=0,\left.\quad \Pi_{0} S\left(\mathbf{V}_{0}\right) \mathbf{n}_{0}\right|_{\Gamma}=0
$$

hold, then the solution of the problem (1.11) satisfies the inequalities

$$
\begin{aligned}
& \sup _{\tau<t}|\mathbf{V}(\cdot, \tau)|_{C^{3+\alpha}(\Gamma)} \leqslant c\left|\mathbf{V}_{0}\right|_{C^{3+\alpha}(\Omega)}, \\
& \sup _{t-\delta<\tau<t}|\mathbf{V}(\cdot, \tau)|_{C^{3+\alpha}(\Gamma)} \leqslant c \varepsilon \delta^{-1}\left(1+\varepsilon \delta^{-1}\right)^{\frac{2 \alpha+7}{4}} \sup _{t-2 \delta<\tau<t}\|\mathbf{V}(\cdot, \tau)\|_{L_{2}(\Omega)},
\end{aligned}
$$

where $\delta(t)$ is the same as in the Proposition 4.6: $\delta=\min \left(\frac{t}{4}, \frac{t_{1}}{4}\right)$.

Proof. We obtain stronger inequalities than (4.22) and (4.23), namely, we estimate in the whole domain $\Omega$ the second derivatives of the functions

$$
\mathbf{V}^{i}=\partial_{i} \mathbf{V}, \quad P^{i}=\partial_{i} P,
$$

where

$$
\partial_{i}=\frac{\partial}{\partial \xi_{i}}-N_{i}(\xi) \sum_{k=1}^{3} N_{k}(\xi) \frac{\partial}{\partial \xi_{k}}
$$

and $N_{i} \in C^{3+\alpha}(\Omega)$ is an extension of $n_{0_{i}} \in C^{3+\alpha}(\Gamma)$ from $\Gamma$ into $\Omega$. For $\xi \in \Gamma$, $\partial_{i}$ are the tangential components of the gradient, therefore $\left.\partial_{i} f\right|_{\Gamma}$ depend only on $\left.f\right|_{\Gamma}$ and the operators $\partial_{i}$ can be applied to functions defined on $\Gamma$.

Applying $\partial_{i}$ to the equations (1.11) we see that $\mathbf{V}^{i}, P^{i}$ are solutions to the problems

$$
\begin{aligned}
& \varepsilon \mathbf{V}_{t}^{i}-\nabla_{w}^{2} \mathbf{V}^{i}+\ell_{w}\left(\mathbf{V}^{i}\right)+\nabla_{w} P^{i}=Q_{1}^{i}(\mathbf{V}, P), \\
& \nabla_{w} \cdot \mathbf{V}^{i}=Q_{2}^{i}(\mathbf{V}), \\
& \left.\mathbf{V}^{i}\right|_{t=0}=\mathbf{V}_{0}^{i}, \\
& \Pi_{w} S_{w}\left(\mathbf{V}^{i}\right) \mathbf{n}_{w}=Q_{3}^{i}(\mathbf{V}), \\
& \mathbf{n}_{w} \cdot T_{w}\left(\mathbf{V}^{i}\right) \mathbf{n}_{w}=Q_{4}^{i}(\mathbf{V}),
\end{aligned}
$$

where

$$
\begin{aligned}
& Q_{1}^{i}(\mathbf{V}, P)=\left(\ell_{w}\left(\mathbf{V}^{i}\right)-\partial_{i} \ell_{w}(\mathbf{V})\right)+\left[\partial_{i}, \nabla_{w}^{2}\right] \mathbf{V}-\left[\partial_{i}, \nabla_{w}\right] P, \\
& Q_{2}^{i}(\mathbf{V})=-\left[\partial_{i}, \nabla_{w}\right] \cdot \mathbf{V}, \\
& Q_{3}^{i}(\mathbf{V})=\Pi_{w}\left(\Pi_{w} S_{w}\left(\partial_{i} \mathbf{V}\right) \mathbf{n}_{w}-\partial_{i}\left(\Pi_{w} S_{w}(\mathbf{V}) \mathbf{n}_{w}\right)\right), \\
& Q_{4}^{i}(\mathbf{V})=\mathbf{n}_{w} \cdot S_{w}\left(\partial_{i} \mathbf{V}\right) \mathbf{n}_{w}-\partial_{i}\left(\mathbf{n}_{w} \cdot S_{w}(\mathbf{V}) \mathbf{n}_{w}\right) .
\end{aligned}
$$

and by $\left[\partial_{i}, \nabla_{w}\right]$ we mean the commutator

$$
\partial_{i} \nabla_{w}-\nabla_{w} \partial_{i}=\left(\partial_{i} A^{(w)}\right) \nabla+\sum_{k=1}^{3} \nabla_{w}\left(N_{i} N_{k}\right) \frac{\partial}{\partial \xi_{k}} \equiv\left(\sum_{k=1}^{3} b_{m k}^{i} \frac{\partial}{\partial \xi_{k}}\right)_{m=1,2,3} .
$$


Moreover, we have

$$
\begin{aligned}
& Q_{2}^{i}(\mathbf{V})=\nabla \cdot \mathbf{R}^{i}+R_{0}^{i}, \\
& R_{k}^{i}=\sum_{m=1}^{3} b_{m k}^{i} V_{m}, \quad R_{0}^{i}=-\sum_{m=1}^{3} \frac{\partial b_{m k}^{i}}{\partial \xi_{k}} V_{m} .
\end{aligned}
$$

It is easy to see that the coefficients of $Q_{j}^{i}$ have the same differentiability properties as the coefficients of $M_{j}$ and that

$$
\begin{gathered}
\sup _{\tau<t}\left|Q_{1}^{i}(\mathbf{V}, P)\right|_{C^{\alpha}(\Omega)}+\sup _{\tau<t}\left|Q_{2}^{i}(\mathbf{V})\right|_{C^{1+\alpha}(\Omega)}+\varepsilon \sup _{\tau<t}\left|\mathbf{R}_{t}^{i}(\cdot, \tau)\right|_{C^{\alpha}(\Omega)} \\
\quad+\varepsilon \sup _{\tau<t} \sup _{\Omega}\left|R_{0 t}^{i}(\xi, \tau)\right|+\sup _{\tau<t}\left|Q_{3}^{i}(\mathbf{V})\right|_{C^{1+\alpha}(\Gamma)}+\sup _{\tau<t}\left|Q_{4}^{i}(\mathbf{V})\right|_{C^{1+\alpha}(\Gamma)}+\varepsilon^{\frac{1+\alpha}{2}} \sup _{\Gamma}\left[Q_{3}^{i}(\mathbf{V})\right]_{(0, t)}^{\left(\frac{1+\alpha}{2}\right)} \\
\quad \leqslant c Y_{t}(\mathbf{V}, P) .
\end{gathered}
$$

Compatibility conditions $\left.Q_{2}^{i}\left(\mathbf{V}_{0}^{i}\right)\right|_{t=0}=\nabla \cdot \mathbf{V}_{0}^{i},\left.Q_{3}^{i}\left(\mathbf{V}_{0}^{i}\right)\right|_{t=0}=\left.\Pi_{0} S\left(\mathbf{V}_{0}^{i}\right) \mathbf{n}_{0}\right|_{\Gamma}$ are easily verified. Hence, in virtue of inequality (4.15),

$$
Y_{t}\left(\mathbf{V}^{i}, P^{i}\right) \leqslant c\left(Y_{t}(\mathbf{V}, P)+\left|\mathbf{V}_{0}^{i}\right|_{C^{2+\alpha}(\Omega)}\right) \leqslant c\left|\mathbf{V}_{0}\right|_{C^{3+\alpha}(\Omega)}
$$

This implies (4.22):

$$
\sup _{\tau<t}|\mathbf{V}(\cdot, \tau)|_{C^{3+\alpha}(\Gamma)} \leqslant c \sum_{i=1}^{3} \sup _{\tau<t}\left|\mathbf{V}^{i}(\cdot, \tau)\right|_{C^{2+\alpha}(\Omega)} \leqslant c\left|\mathbf{V}_{0}\right|_{C^{3+\alpha}(\Omega)} .
$$

In order to obtain (4.23), we proceed as in the proof of Proposition 5.6. We introduce the functions $\mathbf{U}^{i}=V^{i} \omega_{\delta}, Q^{i}=P^{i} \omega_{\delta}$ which satisfy the equations

$$
\begin{aligned}
& \varepsilon \mathbf{U}_{t}^{i}+\ell_{w}\left(\mathbf{U}^{i}\right)-\nabla_{w}^{2} \mathbf{U}^{i}+\nabla_{w} Q^{i}=\varepsilon \mathbf{V}^{i} \omega_{\delta}^{\prime}+Q_{1}^{i}\left(\mathbf{V} \omega_{\delta}, P \omega_{\delta}\right), \\
& \nabla_{w} \cdot \mathbf{U}^{i}=Q_{2}^{i}\left(\mathbf{V} \omega_{\delta}\right), \\
& \left.\mathbf{U}^{i}\right|_{t=0}=0, \\
& \Pi_{w} S_{w}\left(\mathbf{U}^{i}\right) \mathbf{n}_{w}=Q_{3}^{i}\left(\mathbf{V} \omega_{\delta}\right), \\
& \mathbf{n}_{w} \cdot T\left(\mathbf{U}^{i}\right) \mathbf{n}_{w}=Q_{4}^{i}\left(\mathbf{V} \omega_{\delta}\right) .
\end{aligned}
$$

By virtue of (4.15) and (4.25),

$$
\begin{aligned}
& Y_{t_{0}}\left(\mathbf{U}^{i}, Q^{i}\right) \leqslant c\left(Y_{t_{0}}\left(\mathbf{V} \omega_{\delta}, P \omega_{\delta}\right)+\varepsilon \delta^{-1} \sup _{t_{0}-\delta<t<t_{0}}\left|\mathbf{V}^{i}(\xi, t)\right|_{C^{\alpha}(\Omega)}\right) \\
& \leqslant c\left(\varepsilon \sup _{t_{0}-3 \delta / 2<\tau<t_{0}}\left|\mathbf{V}_{\tau}(\cdot, \tau)\right|_{C^{\alpha}(\Omega)}+\sup _{t_{0}-3 \delta / 2<\tau<t_{0}}|\mathbf{V}(\cdot, \tau)|_{C^{2+\alpha}(\Omega)}+\sup _{t_{0}-3 \delta / 2<\tau<t_{0}}|P(\cdot, \tau)|_{C^{1+\alpha}(\Omega)}\right) \\
& \quad+c \varepsilon \delta^{-1} \sup _{t_{0}-3 \delta / 2<\tau<t_{0}}|\mathbf{V}(\xi, t)|_{C^{1+\alpha}(\Omega)} .
\end{aligned}
$$


The right-hand side may be estimated by inequality (4.20) which gives

$$
Y_{t_{0}}\left(\mathbf{U}^{i}, Q^{i}\right) \leqslant c \varepsilon \delta^{-1}\left(1+\varepsilon \delta^{-1}\right)^{\frac{2 \alpha+7}{4}} \sup _{t_{0}-2 \delta<\tau<t}\|\mathbf{V}(\cdot, \tau)\|_{L_{2}(\Omega)}
$$

The proposition is proved.

COROllary 4.2 If $\Gamma \in C^{3+\alpha}, \mathbf{V}_{0} \in C^{2+\alpha}(\Omega)$, then

$$
\int_{0}^{T_{0}}|\mathbf{V}|_{C^{2+\alpha}(\Omega)} \mathrm{d} t+\int_{0}^{T_{0}}|P|_{C^{1+\alpha}(\Omega)} \mathrm{d} t \leqslant c \varepsilon .
$$

If $\Gamma \in C^{4+\alpha}, \mathbf{V}_{0} \in C^{3+\alpha}(\Omega)$, then

$$
\int_{0}^{T_{0}}|\mathbf{V}|_{C^{3+\alpha}(\Gamma)} \mathrm{d} t \leqslant c \varepsilon
$$

Indeed, making use of (1.13) and (4.20) for $t<\varepsilon$ and $t>\varepsilon$, respectively, we obtain

$$
\begin{aligned}
& \int_{0}^{\varepsilon}\left(|\mathbf{V}|_{C^{2+\alpha}(\Omega)}+|P|_{C^{1+\alpha}(\Omega)}\right) \mathrm{d} t+\int_{\varepsilon}^{T_{0}}\left(|\mathbf{V}|_{C^{2+\alpha}(\Omega)}+|P|_{C^{1+\alpha}(\Omega)}\right) \mathrm{d} t \\
& \quad \leqslant c\left(\varepsilon\left|\mathbf{V}_{0}\right|_{C^{2+\alpha}(\Omega)}+\left\|\mathbf{V}_{0}\right\|_{L_{2}(\Omega)} \int_{\varepsilon}^{T_{0}} \varepsilon \delta^{-1}(t)\left(1+\varepsilon \delta^{-1}(t)\right)^{\frac{2 \alpha+3}{4}} e^{-\frac{b t}{\varepsilon}} \mathrm{d} t\right) \\
& \quad \leqslant c \varepsilon\left|\mathbf{V}_{0}\right|_{C^{2+\alpha}(\Omega)} .
\end{aligned}
$$

The proof of (4.27) is similar.

\section{Proof of Theorems 3 and 4}

\subsection{Proof of Theorem 3}

At first we consider the problem (1.12) with a modified boundary condition, namely,

$$
\begin{aligned}
& \varepsilon \boldsymbol{\eta}_{t}-\nabla_{w}^{2} \boldsymbol{\eta}+\ell_{w}(\boldsymbol{\eta})+\nabla_{w} \pi=\mathbf{f}, \\
& \nabla_{w} \cdot \boldsymbol{\eta}=g, \\
& \left.\boldsymbol{\eta}\right|_{t=0}=0, \\
& \left.\Pi_{w} S_{w}(\boldsymbol{\eta}) \mathbf{n}_{w}\right|_{\Gamma}=\mathbf{b}, \\
& \mathbf{n}_{w} \cdot T_{w}(\boldsymbol{\eta}, \pi) \mathbf{n}_{w}-\left.\int_{0}^{t} \mathbf{n}_{w}(\xi, \tau) \cdot \Delta_{w}(\tau) \boldsymbol{\eta} \mathrm{d} \tau\right|_{\Gamma}=b+\int_{0}^{t} B \mathrm{~d} \tau
\end{aligned}
$$


and prove its solvability on a certain small but fixed time interval $\left(0, t_{2}\right)$. We write $(5.1)$ in the form $\varepsilon \boldsymbol{\eta}_{t}-\nabla^{2} \boldsymbol{\eta}+\ell(\boldsymbol{\eta})+\nabla \pi=\mathbf{f}+M_{1}(\boldsymbol{\eta}, \pi)$,

$\nabla \cdot \boldsymbol{\eta}=g+M_{2}(\boldsymbol{\eta})$,

$\left.\eta\right|_{t=0}=0$,

$\left.\Pi_{0} S(\boldsymbol{\eta}) \mathbf{n}_{0}\right|_{\Gamma}=\Pi_{0} \mathbf{b}+M_{3}(\boldsymbol{\eta})$,

$\mathbf{n}_{0} \cdot T(\boldsymbol{\eta}, \pi) \mathbf{n}_{0}-\left.\mathbf{n}_{0} \cdot \Delta_{0} \int_{0}^{t} \boldsymbol{\eta} d \boldsymbol{\eta}\right|_{\Gamma}=b+\int_{0}^{t} B \mathrm{~d} \tau+M_{4}(\boldsymbol{\eta})+\int_{0}^{t} M_{5}(\boldsymbol{\eta}) \mathrm{d} \tau$,

where $M_{1}-M_{4}$ are defined above in (4.16), and

$$
M_{5}(\boldsymbol{\eta})=\left(\mathbf{n}_{0} \cdot \Delta_{0}-\mathbf{n}_{w} \cdot \Delta_{w}(t)\right) \boldsymbol{\eta} .
$$

Problems (5.1) and (5.2) are equivalent if $\mathbf{n}_{w} \cdot \mathbf{n}_{0}>0$ which holds for small $t$.

By virtue of (4.6)-(4.8), there holds the estimate

$$
\begin{array}{r}
\sup _{\tau<t}\left|M_{1}(\boldsymbol{\eta}, \pi)\right|_{C^{\alpha}(\Omega)}+\sup _{\tau<t}\left|M_{2}(\boldsymbol{\eta})\right|_{C^{1+\alpha}(\Omega)}+\varepsilon \sup _{\tau<t}\left|H_{t}(\boldsymbol{\eta})\right|_{C^{\alpha}(\Omega)}+\sup _{\tau<t}\left|M_{3}(\boldsymbol{\eta})\right|_{C^{1+\alpha}(\Gamma)} \\
+\varepsilon^{\frac{1+\alpha}{2}} \sup _{\Gamma}\left[M_{3}(\boldsymbol{\eta})\right]_{(0, t)}^{\left(\frac{1+\alpha}{2}\right)}+\sup _{\tau<t}\left|M_{4}(\boldsymbol{\eta})\right|_{C^{1+\alpha}(\Gamma)}+\sup _{\tau<t}\left|M_{5}(\boldsymbol{\eta})\right|_{C^{\alpha}(\Gamma)} \\
\leqslant c_{1}\left(\int_{0}^{t}|\mathbf{w}(\cdot, \tau)|_{C^{2+\alpha}(\Omega)} \mathrm{d} \tau+\varepsilon \sup _{\tau<t}|\mathbf{w}(\cdot, \tau)|_{C^{2+\alpha}(\Omega)}\right)\left(\varepsilon \sup _{\tau<t}\left|\boldsymbol{\eta}_{\tau}(\cdot, \tau)\right|_{C^{\alpha}(\Omega)}\right. \\
\left.+\sup _{\tau<t}|\boldsymbol{\eta}(\cdot, \tau)|_{C^{2+\alpha}(\Omega)}+\sup _{\tau<t}|\pi(\cdot, \tau)|_{C^{1+\alpha}(\Omega)}\right) .
\end{array}
$$

Therefore, if $t_{2}$ is so small that

$$
c_{1} c_{2}\left(\int_{0}^{t_{2}}|\mathbf{w}(\cdot, \tau)|_{C^{2+\alpha}(\Omega)} \mathrm{d} \tau+\varepsilon \sup _{\tau<t_{2}}|\mathbf{w}(\cdot, \tau)|_{C^{2+\alpha}(\Omega)}\right) \leqslant \frac{1}{2},
$$

where $c_{2}$ is a constant in the inequality (1.23), then the solvability ot the problem (5.1) can be established by the method of successive approximations or deduced from the contraction mapping principle. Inequality (1.23) and condition (5.3) imply (1.17) for $t<t_{2}$.

To extend the solution outside the interval $\left(0, t_{2}\right)$ we introduce a smooth monotone function $\chi_{1}(t)$ such that $\chi_{1}(t)=1$ for $t<t_{2} / 3, \chi_{1}(t)=0$ for $t_{1}>2 t_{2} / 3$, and we set $\chi_{2}(t)=1-\chi_{1}(t)$. It can be easily verified that $\boldsymbol{\eta} \chi_{i} \equiv \boldsymbol{\eta}_{i}, \pi \chi_{i} \equiv \pi_{i}$ satisfy the equations

$$
\begin{aligned}
& \varepsilon \boldsymbol{\eta}_{i t}-\nabla_{w}^{2} \boldsymbol{\eta}_{i}+\ell_{w}\left(\boldsymbol{\eta}_{i}\right)+\nabla_{w} \pi_{i}=\mathbf{f} \chi_{i}+\varepsilon \boldsymbol{\eta} \chi_{i}^{\prime}, \\
& \nabla_{w} \cdot \boldsymbol{\eta}_{i}=g \chi_{i}, \\
& \left.\boldsymbol{\eta}_{i}\right|_{t=0}=0, \\
& \left.\Pi_{w} S_{w}\left(\boldsymbol{\eta}_{i}\right) \mathbf{n}_{w}\right|_{\Gamma}=\mathbf{b} \chi_{i},
\end{aligned}
$$




$$
\begin{aligned}
\mathbf{n}_{w} \cdot T_{w}\left(\boldsymbol{\eta}_{i}, \pi_{i}\right) \mathbf{n}_{w}-\left.\int_{0}^{t} \mathbf{n}_{w} \cdot \Delta_{w}(\tau) \boldsymbol{\eta}_{i} \mathrm{~d} \tau\right|_{\Gamma} \\
=\int_{0}^{t}\left(\left.\mathbf{n}_{w} \cdot T_{w}(\boldsymbol{\eta}, \pi) \mathbf{n}_{w}\right|_{\Gamma}-b\right) \chi_{i}^{\prime} \mathrm{d} \tau+\int_{0}^{t} B \chi_{i} \mathrm{~d} \tau+b \chi_{i} .
\end{aligned}
$$

The functions $\eta_{2}, \pi_{2}$ vanish for $t \in\left(0, t_{2} / 3\right)$, so $\eta_{2}$ satisfies the condition

$$
\left.\eta_{2}\right|_{t=t_{2} / 3}=0
$$

hence, integration in (5.7) in the case $i=2$ is carried out in the limits $\tau \in\left(t_{2} / 3, t\right)$ (in the first integral of the right-hand side, in the limits $\left.\tau \in\left(t_{2} / 3, \min \left(t, 2 t_{2} / 3\right)\right)\right)$. In order to show that the solution $\left(\boldsymbol{\eta}_{2}, \pi_{2}\right)$ of problem (5.4)-(5.8), $i=2$, is defined for $t \in\left(t_{2} / 3,4 t_{2} / 3\right)$, we make the change of variables

$$
\xi^{\prime}=X_{w}\left(\xi, t_{2} / 3\right) \equiv X_{w}(\xi)
$$

and write this problem in the form

$$
\begin{aligned}
& \varepsilon \boldsymbol{\eta}_{2 t}^{\prime}-\nabla_{w}^{\prime 2} \boldsymbol{\eta}_{2}^{\prime}+\ell_{w}^{\prime}\left(\boldsymbol{\eta}_{2}^{\prime}\right)+\nabla_{w}^{\prime} \pi_{2}^{\prime}=\mathbf{f}_{2}, \\
& \nabla_{w}^{\prime} \cdot \boldsymbol{\eta}_{2}^{\prime}=g_{2}, \quad \xi^{\prime} \in \Omega_{t_{1} / 3} \equiv \Omega^{\prime}, \quad t>t_{1} / 3, \\
& \left.\boldsymbol{\eta}_{2}^{\prime}\right|_{t=t_{1} / 3}=0, \\
& \left.\Pi_{w}^{\prime} S_{w}^{\prime}\left(\boldsymbol{\eta}_{2}^{\prime}\right) \mathbf{n}_{w}^{\prime}\right|_{\Gamma^{\prime}}=\mathbf{b}_{2}, \\
& \mathbf{n}_{w}^{\prime} \cdot T_{w}^{\prime}\left(\boldsymbol{\eta}_{2}^{\prime}, \pi_{2}^{\prime}\right) \mathbf{n}_{w}^{\prime}-\left.\int_{t_{1} / 3}^{t} \mathbf{n}_{w}^{\prime} \cdot \Delta_{w}^{\prime}(\tau) \boldsymbol{\eta}_{2}^{\prime} \mathrm{d} \tau\right|_{\Gamma^{\prime}}=b_{2}+\int_{t_{1} / 3}^{t} B_{2} \mathrm{~d} \tau,
\end{aligned}
$$

(cf. (4.18)) where

$$
\begin{gathered}
\Gamma^{\prime}=\partial \Omega^{\prime}, \quad \boldsymbol{\eta}_{2}^{\prime}=\boldsymbol{\eta}_{2} \circ X_{w}^{-1}, \quad \pi_{2}^{\prime}=\pi_{2} \circ X_{w}^{-1}, \quad \mathbf{f}_{2}=\left(\mathbf{f} \chi_{2}+\varepsilon \boldsymbol{\eta} \chi_{2}^{\prime}\right) \circ X_{w}^{-1}, \\
g_{2}=g \chi_{2} \circ X_{w}^{-1}, \quad \mathbf{b}_{2}=\mathbf{b} \chi_{2} \circ X_{w}^{-1}, \quad B_{2}=B \chi_{2} \circ X_{w}^{-1}, \\
b_{2}=\left[\int_{t_{1} / 3}^{t}\left(\left.\mathbf{n}_{w} \cdot T_{w}(\boldsymbol{\eta}, \pi) \mathbf{n}_{w}\right|_{\Gamma}-b\right) \chi_{2}^{\prime} \mathrm{d} \tau+b \chi_{2}\right] \circ X_{w}^{-1} .
\end{gathered}
$$

Problem (5.9) has exactly the same form as (5.1), so by virtue of uniform estimate (4.10) it is solvable in the interval $t \in\left(t_{2} / 3,4 t_{2} / 3\right)$ of the length $t_{2}$ and the solution satisfies the estimate of the type (1.17):

$$
\begin{aligned}
& \varepsilon \sup _{\tau \in\left(t_{2} / 3, t\right)}\left|\boldsymbol{\eta}_{2 \tau}^{\prime}(\cdot, \tau)\right|_{C^{\alpha}\left(\Omega^{\prime}\right)}+\sup _{\tau \in\left(t_{2} / 3, t\right)}\left|\boldsymbol{\eta}_{2}^{\prime}(\cdot, \tau)\right|_{C^{2+\alpha}\left(\Omega^{\prime}\right)}+\sup _{\tau \in\left(t_{2} / 3, t\right)}\left|\pi_{2}^{\prime}(\cdot, \tau)\right|_{C^{1+\alpha}\left(\Omega^{\prime}\right)} \leqslant \\
& c\left(\sup _{\tau \in\left(t_{2} / 3, t\right)}\left|\mathbf{f}_{2}(\cdot, \tau)\right|_{C^{\alpha}\left(\Omega^{\prime}\right)}+\sup _{\tau \in\left(t_{2} / 3, t\right)}\left|g_{2}(\cdot, \tau)\right|_{C^{1+\alpha}\left(\Omega^{\prime}\right)}+\sup _{\tau \in\left(t_{2} / 3, t\right)}\left|\mathbf{h}_{2 \tau}(\cdot, \tau)\right|_{C^{\alpha}\left(\Omega^{\prime}\right)}\right. \\
& \quad+\sup _{\tau \in\left(t_{2} / 3, t\right)} \sup _{\Omega^{\prime}}\left|h_{20 \tau}\left(\xi^{\prime}, \tau\right)\right|+\sup _{\tau \in\left(t_{2} / 3, t\right)}\left|\mathbf{b}_{2}(\cdot, \tau)\right|_{C^{1+\alpha}\left(\Gamma^{\prime}\right)}+\varepsilon^{\frac{1+\alpha}{2}} \sup _{\Gamma^{\prime}}\left[\mathbf{b}_{2}\right]_{\left(t_{2} / 3, t\right)}^{\left(\frac{1+\alpha}{2}\right)}
\end{aligned}
$$




$$
\left.+\sup _{\tau \in\left(t_{2} / 3\right), t}\left|b_{2}(\cdot, \tau)\right|_{C^{1+\alpha}\left(\Gamma^{\prime}\right)}+\sup _{\tau \in\left(t_{2} / 3, t\right)}|B(\cdot, \tau)|_{C^{\alpha}\left(\Gamma^{\prime}\right)}\right), \quad t \in\left(t_{2} / 3,4 t_{2} / 3\right),
$$

where $\mathbf{h}_{2}=\mathbf{h} \chi_{2} \circ X_{w}^{-1}, h_{02}=h_{0} \chi_{2} \circ X_{w}^{-1}$. Clearly, $\boldsymbol{\eta}=\boldsymbol{\eta} \chi_{1}+\boldsymbol{\eta}_{2}, \pi=\pi \chi_{1}+\pi_{2}$ is a solution of (5.1) in the interval $\left(0,4 t_{2} / 3\right)$.

Estimate (1.17) for $t \in\left(0,4 t_{2} / 3\right)$ follows from (5.10) and from (1.17), already proved for $t<t_{2}$. This argument can be repeated till the solution of (5.1) is extended into the interval $t \in\left(0, T_{0}\right)$. Now, we consider the problem (1.12). The last boundary condition can be written in the form

$$
\mathbf{n}_{w} \cdot T_{w}(\boldsymbol{\eta}, \pi) \mathbf{n}_{w}-\left.\int_{0}^{t} \mathbf{n}_{w}(\xi, \tau) \cdot \Delta_{w}(\tau) \boldsymbol{\eta}(\xi, \tau) \mathrm{d} \tau\right|_{\Gamma}=b+\int_{0}^{t} B \mathrm{~d} \tau+\int_{0}^{t} Q(\boldsymbol{\eta}) \mathrm{d} \tau
$$

where

$$
Q(\boldsymbol{\eta})=\left[\dot{\mathbf{n}}_{w}(\xi, t) \cdot \Delta_{w}(t)+\mathbf{n}_{w}(\xi, t) \cdot \dot{\Delta}_{w}(t)\right] \int_{0}^{t} \boldsymbol{\eta}(\xi, \tau) \mathrm{d} \tau
$$

Since

$$
\sup _{\tau<t}|Q(\boldsymbol{\eta})|_{C^{\alpha}(\Gamma)} \leqslant c \int_{0}^{t}|\boldsymbol{\eta}(\cdot, \tau)|_{C^{2+\alpha}(\Gamma)} \mathrm{d} \tau,
$$

we can use the theorem on the solvability of problem (5.1) which has been just proved to obtain the solution of (1.12) by the method of successive approximations.

This completes the proof of Theorem 5 .

\subsection{Proof of Theorem 4}

We find the solution of problem (1.19) by the method of successive approximations. At zero approximation, we take $\boldsymbol{\eta}_{0}=0, \pi_{0}=0$ and we define $\eta_{m+1}, \pi_{m+1} m=0,1, \ldots$ as solutions to the problems

$$
\begin{aligned}
& \varepsilon\left(\boldsymbol{\eta}_{m+1}\right)_{t}-\nabla_{w}^{2} \boldsymbol{\eta}_{m+1}+\ell_{w}\left(\boldsymbol{\eta}_{m+1}\right)+\nabla_{w} \pi_{m+1}=-\varepsilon \mathbf{w}_{t}+\mathcal{L}_{1}\left(\boldsymbol{\eta}_{m}, \pi_{m}\right), \\
& \nabla_{w} \cdot \boldsymbol{\eta}_{m+1}=\mathcal{L}_{2}\left(\boldsymbol{\eta}_{m}\right), \\
& \left.\boldsymbol{\eta}_{m+1}\right|_{t=0}=0, \\
& \left.\Pi_{w} S_{w}\left(\boldsymbol{\eta}_{m+1}\right) \mathbf{n}_{w}\right|_{\Gamma}=\mathcal{L}_{3}\left(\boldsymbol{\eta}_{m}\right), \\
& \mathbf{n}_{w} \cdot T_{w}\left(\boldsymbol{\eta}_{m+1}, \pi_{m+1}\right) \mathbf{n}_{w}-\left.\mathbf{n}_{w} \cdot \Delta_{w}\left(X_{m+1}-X_{w}\right)\right|_{\Gamma}=\mathcal{L}\left(\boldsymbol{\eta}_{m}, \pi_{m}\right),
\end{aligned}
$$


where $\mathcal{L}_{i}, \mathcal{L}$ are defined in Section $1, X_{m} \equiv X_{u_{m}}$. For $m=0$ we have

$$
\begin{aligned}
& \varepsilon \boldsymbol{\eta}_{1 t}-\nabla_{w}^{2} \boldsymbol{\eta}_{1}+\ell_{w}\left(\boldsymbol{\eta}_{1}\right)+\nabla_{w} \pi_{1}=-\varepsilon \mathbf{w}_{t}+\left(\nabla_{u_{0}}^{2}-\nabla_{w}^{2}\right) \mathbf{u}_{0}-\left(\nabla_{u_{0}}-\nabla_{w}\right) q_{0} \\
& +\ell_{w}\left(\mathbf{u}_{0}\right)-\ell_{u_{0}}\left(\mathbf{u}_{0}\right)+\sum_{k=1}^{6} \mu_{k}\left(\boldsymbol{\varphi}_{k}\left(X_{u_{0}}(\xi, t)\right)-\boldsymbol{\varphi}_{k}\left(X_{w}(\xi, t)\right)\right) \equiv \mathbf{f}_{0}(\xi, t), \\
& \nabla_{w} \cdot \boldsymbol{\eta}_{1}=\left(\nabla_{w}-\nabla_{u_{0}}\right) \cdot \mathbf{u}_{0} \equiv g_{0}(\xi, t), \\
& \left.\eta_{1}\right|_{t=0}=0 \text {, } \\
& \left.\Pi_{w} S\left(\boldsymbol{\eta}_{1}\right) \mathbf{n}_{w}\right|_{\Gamma}=\left.\Pi_{w}\left(\Pi_{w} S_{w}\left(\mathbf{u}_{0}\right) \mathbf{n}_{w}-\Pi_{u_{0}} S_{u_{0}}\left(\mathbf{u}_{0}\right) \mathbf{n}_{u_{0}}\right)\right|_{\Gamma} \equiv \mathbf{b}_{0}(\xi, t), \\
& \mathbf{n}_{w} \cdot T_{w}\left(\boldsymbol{\eta}_{1}, \pi_{1}\right) \mathbf{n}_{w}-\left.\mathbf{n}_{w} \cdot \Delta_{w}(t) \int_{0}^{t} \boldsymbol{\eta}_{1} \mathrm{~d} \tau\right|_{\Gamma} \\
& =\left.\mathbf{n}_{w} \cdot\left(T_{w}\left(\mathbf{u}_{0}, q_{0}\right) \mathbf{n}_{w}-T_{u_{0}}\left(\mathbf{u}_{0}, q_{0}\right) \mathbf{n}_{u_{0}}\right)\right|_{\Gamma}+\mathbf{n}_{w} \cdot\left(\Delta_{u_{0}}(t)-\Delta_{w}(t)\right) \mathbf{X}_{u_{0}} \\
& +\left.\mathbf{n}_{w} \cdot \Delta_{w}(t) \int_{0}^{t} \mathbf{V}(\xi, t) \mathrm{d} \tau\right|_{\Gamma} \equiv b_{0}(\xi, t) \\
& \text { where } \mathbf{u}_{0}=\mathbf{w}+\mathbf{V}, q_{0}=r+P \text {. } \\
& g_{0}=\nabla \cdot \mathbf{h}_{0}, \quad \mathbf{h}_{0}=\left(\mathcal{A}^{(w)}-\mathcal{A}^{\left(u_{0}\right)}\right)^{T} \mathbf{u}_{0} .
\end{aligned}
$$

By virtue of (4.6)-(4.8), we have

$$
\begin{aligned}
& \sup _{t<T_{0}}\left|\mathbf{f}_{0}(\cdot, t)\right|_{C^{\alpha}(\Omega)}+\sup _{t<T_{0}}\left|g_{0}(\cdot, t)\right|_{C^{1+\alpha}(\Omega)}+\sup _{t<T_{0}}\left|\mathbf{h}_{0 t}(\cdot, t)\right|_{C^{\alpha}(\Omega)} \\
& +\sup _{\tau<T_{0}}\left|\mathbf{b}_{0}(\cdot, t)\right|_{C^{1+\alpha}(\Gamma)}+\sup _{\tau<T_{0}}\left|b_{0}(\cdot, t)\right|_{C^{1+\alpha}(\Gamma)}+\varepsilon^{\frac{1+\alpha}{2}} \sup _{\Gamma}\left[\mathbf{b}_{0}\right]_{\left(0, T_{0}\right)}^{\left(\frac{1+\alpha}{2}\right)} \leqslant c \varepsilon,
\end{aligned}
$$

hence, making use of the inequality (1.17), we obtain

$$
\varepsilon \sup _{t<T_{0}}\left|\eta_{1 t}(\cdot, t)\right|_{C^{\alpha}(\Omega)}+\sup _{t<T_{0}}\left|\boldsymbol{\eta}_{1}(\cdot, t)\right|_{C^{2+\alpha}(\Omega)}+\sup _{t<T_{0}}\left|\pi_{1}(\cdot, t)\right|_{C^{1+\alpha}(\Omega)} \leqslant c \varepsilon .
$$

The functions $\psi_{m+1}=\boldsymbol{\eta}_{m+1}-\boldsymbol{\eta}_{m}, \rho_{m+1}=\pi_{m+1}-\pi_{m}$ satisfy the equations

$$
\begin{aligned}
& \varepsilon\left(\boldsymbol{\psi}_{m+1}\right)_{t}-\nabla_{w}^{2} \boldsymbol{\psi}_{m+1}+\ell_{w}\left(\boldsymbol{\psi}_{m+1}\right)+\nabla_{w} \rho_{m+1}=\mathcal{L}_{1}\left(\boldsymbol{\eta}_{m}, \pi_{m}\right)-\mathcal{L}_{1}\left(\boldsymbol{\eta}_{m-1}, \pi_{m-1}\right), \\
& \nabla_{w} \cdot \boldsymbol{\psi}_{m+1}=\mathcal{L}_{2}\left(\boldsymbol{\eta}_{m}\right)-\mathcal{L}_{2}\left(\boldsymbol{\eta}_{m-1}\right), \\
& \left.\boldsymbol{\psi}_{m+1}\right|_{t=0}=0, \\
& \left.\Pi_{w} S_{w}\left(\boldsymbol{\psi}_{m+1}\right) \mathbf{n}_{w}\right|_{\Gamma}=\mathcal{L}_{3}\left(\boldsymbol{\eta}_{m}\right)-\mathcal{L}_{3}\left(\boldsymbol{\eta}_{m-1}\right), \\
& \mathbf{n}_{w} \cdot T_{w}\left(\boldsymbol{\psi}_{m+1}, \rho_{m}\right) \mathbf{n}_{w}-\left.\mathbf{n}_{w} \cdot \Delta_{w}(t) \int_{0}^{t} \boldsymbol{\psi}_{m+1} \mathrm{~d} \tau\right|_{\Gamma}=\mathcal{L}\left(\boldsymbol{\eta}_{m}, \pi_{m}\right)-\mathcal{L}\left(\boldsymbol{\eta}_{m-1}, \pi_{m-1}\right) .
\end{aligned}
$$


Setting $\nabla_{u_{m}} \equiv \nabla_{m}, X_{u_{m}} \equiv X_{m}, \Pi_{u_{m}} \equiv \Pi_{m}, S_{u_{m}} \equiv S_{m}, \mathbf{n}_{u_{m}} \equiv \mathbf{n}_{m}$ we easily show that

$$
\begin{aligned}
& \mathcal{L}_{1}\left(\boldsymbol{\eta}_{m}, \pi_{m}\right)-\mathcal{L}_{1}\left(\boldsymbol{\eta}_{m-1}, \pi_{m-1}\right)=\left(\nabla_{m}^{2}-\nabla_{w}^{2}\right) \boldsymbol{\psi}_{m}-\left(\nabla_{m}-\nabla_{w}\right) \rho_{m} \\
& \quad+\left(\ell_{w}\left(\boldsymbol{\psi}_{m}\right)-\ell_{u_{m}}\left(\boldsymbol{\psi}_{m}\right)\right)+\left(\ell_{u_{m-1}}\left(\mathbf{u}_{m-1}\right)-\ell_{u_{m}}\left(\mathbf{u}_{m-1}\right)\right) \\
& \quad+\left(\nabla_{m}^{2}-\nabla_{m-1}^{2}\right) \mathbf{u}_{m-1}-\left(\nabla_{m}-\nabla_{m-1}\right) q_{m-1}+\sum_{k=1}^{6} \mu_{k}\left(\varphi_{k}\left(\mathbf{X}_{m}\right)-\varphi_{k}\left(\mathbf{X}_{m-1}\right)\right), \\
& \mathcal{L}_{2}\left(\boldsymbol{\eta}_{m}\right)-\mathcal{L}_{2}\left(\boldsymbol{\eta}_{m-1}\right)=\left(\nabla_{w}-\nabla_{m}\right) \cdot \boldsymbol{\psi}_{m}+\left(\nabla_{m-1}-\nabla_{m}\right) \cdot \mathbf{u}_{m-1}=\nabla \cdot \overrightarrow{\mathcal{H}}_{m}, \\
& \overrightarrow{\mathcal{H}}_{m}=\left(\mathcal{A}^{(w)}-\mathcal{A}^{(m)}\right)^{T} \boldsymbol{\psi}_{m}+\left(\mathcal{A}^{(m-1)}-\mathcal{A}^{(m)}\right)^{T} \mathbf{u}_{m-1}, \\
& \mathcal{L}_{3}\left(\boldsymbol{\eta}_{m}\right)-\mathcal{L}_{3}\left(\boldsymbol{\eta}_{m-1}\right)=\Pi_{w}\left(\Pi_{w} S_{w}\left(\boldsymbol{\psi}_{m}\right) \mathbf{n}_{w}-\Pi_{m} S_{m}\left(\boldsymbol{\psi}_{m}\right) \mathbf{n}_{m}\right) \\
& \quad+\Pi_{w}\left(\Pi_{m-1} S_{m-1}\left(\mathbf{u}_{m-1}\right) \mathbf{n}_{m-1}-\Pi_{m} S_{m}\left(\mathbf{u}_{m-1}\right) \mathbf{n}_{m}\right), \\
& \mathcal{L}\left(\boldsymbol{\eta}_{m}, \pi_{m}\right)-\mathcal{L}\left(\boldsymbol{\eta}_{m-1}, \pi_{m-1}\right)=\mathcal{P}_{m}+\int_{0}^{t} \mathcal{Q}_{m}^{\prime} \mathrm{d} \tau+\int_{0}^{t} \mathcal{Q}_{m}^{\prime \prime} \mathrm{d} \tau,
\end{aligned}
$$

where

$$
\begin{gathered}
\mathcal{P}_{m}=\mathbf{n}_{w} \cdot\left(T_{w}\left(\boldsymbol{\psi}_{m}, \rho_{m}\right) \mathbf{n}_{w}-T_{m}\left(\boldsymbol{\psi}_{m}, \rho_{m}\right) \mathbf{n}_{m}\right) \\
\quad+\mathbf{n}_{w} \cdot\left(T_{m-1}\left(\mathbf{u}_{m-1}, q_{m-1}\right) \mathbf{n}_{m-1}-T_{m}\left(\mathbf{u}_{m-1}, q_{m-1}\right) \mathbf{n}_{m}\right) \\
\quad+\mathbf{n}_{0} \cdot\left(\Delta_{m}-\Delta_{m-1}\right) \boldsymbol{\xi} \\
\mathcal{Q}_{m}^{\prime}=\left[\dot{\mathbf{n}}_{w} \cdot\left(\Delta_{m}-\Delta_{w}\right)+\mathbf{n}_{w} \cdot\left(\dot{\Delta}_{m}-\dot{\Delta}_{w}\right)\right] \int_{0}^{t} \boldsymbol{\psi}_{m} \mathrm{~d} \tau+\mathbf{n}_{w} \cdot\left(\Delta_{m}-\Delta_{w}\right) \boldsymbol{\psi}_{m} \\
+\left[\dot{\mathbf{n}}_{w}\left(\Delta_{m}-\Delta_{m-1}\right)\left(\boldsymbol{\xi}+\int_{0}^{t} \mathbf{u}_{m-1} \mathrm{~d} \tau\right)+\mathbf{n}_{w}\left(\Delta_{m}-\Delta_{m-1}\right) \mathbf{u}_{m-1}\right] \\
\mathcal{Q}_{m}^{\prime \prime}=\left(\mathbf{n}_{w}-\mathbf{n}_{0}\right)\left(\dot{\Delta}_{m}-\dot{\Delta}_{m-1}\right) \xi+\mathbf{n}_{w} \cdot\left(\dot{\Delta}_{m}-\dot{\Delta}_{m-1}\right) \int_{0}^{t} \mathbf{u}_{m-1} \mathrm{~d} \tau
\end{gathered}
$$

Assume that for $t \leqslant T_{1}$

$$
\begin{gathered}
\varepsilon \sup _{\tau<t}\left|\mathbf{u}_{m \tau}(\cdot, \tau)\right|_{C^{\alpha}(\Omega)}+\sup _{\tau<t}\left|\mathbf{u}_{m}(\cdot, \tau)\right|_{C^{2+\alpha}(\Omega)}++\sup _{\tau<t}\left|q_{m}(\cdot, \tau)\right|_{C^{1+\alpha}(\Omega)} \leqslant c_{0}, \\
\int_{0}^{t}\left|\mathbf{u}_{m}(\cdot, \tau)\right|_{C^{2+\alpha}(\Omega)} \mathrm{d} \tau \leqslant \theta, \quad m=1, \ldots, n,
\end{gathered}
$$

where $\theta$ is the same number as in Proposition 4.2. Then all the problems (5.11), $m=1, \ldots, n$, are solvable in the interval $\left(0, T_{1}\right)$. The norms

$$
Y_{m}(t)=\varepsilon \sup _{\tau<t}\left|\psi_{m \tau}(\cdot, \tau)\right|_{C^{\alpha}(\Omega)}+\sup _{\tau<t}\left|\psi_{m}(\cdot, \tau)\right|_{C^{2+\alpha}(\Omega)}+\sup _{\tau<t}\left|\rho_{m}(\cdot, \tau)\right|_{C^{1+\alpha}(\Omega)}
$$


can be estimated by inequality (1.17) as follows:

$$
\begin{aligned}
Y_{m+1}(t) \leqslant & c\left(\sup _{\tau<t}\left|\mathcal{L}_{1}\left(\boldsymbol{\eta}_{m}, \pi_{m}\right)-\mathcal{L}_{1}\left(\boldsymbol{\eta}_{m-1}, \pi_{m-1}\right)\right|_{C^{\alpha}(\Omega)}+\sup _{\tau<t}\left|\mathcal{L}_{2}\left(\boldsymbol{\eta}_{m}\right)-\mathcal{L}_{2}\left(\boldsymbol{\eta}_{m-1}\right)\right|_{C^{1+\alpha}(\Omega)}\right. \\
& +\varepsilon \sup _{\tau<t}\left|\overrightarrow{\mathcal{H}}_{m \tau}\right| C^{\alpha}(\Omega)+\sup _{\tau<t}\left|\mathcal{L}_{3}\left(\boldsymbol{\eta}_{m}\right)-\mathcal{L}_{3}\left(\boldsymbol{\eta}_{m-1}\right)\right|_{C^{1+\alpha}(\Gamma)} \\
& +\varepsilon^{\frac{1+\alpha}{2}} \sup _{\Gamma}\left[\mathcal{L}_{3}\left(\boldsymbol{\eta}_{m}\right)-\mathcal{L}_{3}\left(\boldsymbol{\eta}_{m-1}\right)\right]_{(0, t)}^{\left(\frac{1+\alpha}{2}\right)}+\sup _{\tau<t}\left|\mathcal{P}_{m}\right|_{C^{1+\alpha}(\Gamma)}+\sup _{\tau<t}\left|Q_{m}^{\prime}\right| C^{\alpha}(\Gamma) \\
& \left.+\sup _{\tau<t}\left|Q_{m}^{\prime \prime}\right| C^{\alpha}(\Gamma)\right) .
\end{aligned}
$$

Consider the last term in (5.12), using local coordinates (see Subsection 4.1). Since $\mathbf{n}_{0} \cdot \frac{\partial \boldsymbol{\xi}}{\partial \eta_{\alpha}}=0$, the terms with $h_{\beta}(\eta, t)$ drop out, and we have, by virtue of Proposition 4.2:

$$
\left|\mathbf{n}_{0} \cdot\left(\Delta_{m}-\Delta_{m-1}\right) \xi\right|_{C^{1+\alpha}(\Gamma)} \leqslant c \int_{0}^{t}\left|\psi_{m}(\cdot, \tau)\right|_{C^{2+\alpha}(\Omega)} \mathrm{d} \tau \leqslant c \int_{0}^{t} Y_{m}(\tau) \mathrm{d} \tau .
$$

Making use of (4.6), (4.7), (4.9), and (4.26), we obtain further

$$
\sup _{\tau<t}\left|\mathcal{Q}_{m}^{\prime \prime}\right|_{C^{\alpha}(\Gamma)} \leqslant c\left(\int_{0}^{t}\left|\boldsymbol{\eta}_{m-1}(\cdot, \tau)\right|_{C^{2+\alpha}(\Omega)} \mathrm{d} \tau+\int_{0}^{t}|\mathbf{w}(\cdot, \tau)|_{C^{2+\alpha}(\Omega)} \mathrm{d} \tau+\varepsilon\right)\left|\psi_{m}(\cdot, \tau)\right|_{C^{2+\alpha}(\Omega)}
$$

Other terms in (5.11) can be easily estimated with the help of the same inequalities by

$$
c\left(\int_{0}^{t}\left|\boldsymbol{\eta}_{m-1}(\cdot, \tau)\right|_{C^{2+\alpha}(\Omega)} \mathrm{d} \tau+\varepsilon\right) Y_{m}(t)+c \int_{0}^{t} Y_{m}(\tau) \mathrm{d} \tau .
$$

Putting all the estimates together we obtain

$$
\begin{aligned}
Y_{m+1}(t) \leqslant & c_{1}\left(\int_{0}^{t}\left(\left|\boldsymbol{\eta}_{m}(\cdot, \tau)\right|_{C^{2+\alpha}(\Omega)}+\left|\boldsymbol{\eta}_{m-1}(\cdot, \tau)\right|_{C^{2+\alpha}(\Omega)}+|\mathbf{w}(\cdot, \tau)|_{C^{2+\alpha}(\Omega)}\right) \mathrm{d} \tau+\varepsilon\right) Y_{m}(t) \\
& +c_{1} \int_{0}^{t} Y_{m}(\tau) \mathrm{d} \tau
\end{aligned}
$$

Let

$$
\Sigma_{n}(t)=\sum_{m=1}^{n} Y_{m}(t)
$$

Since $\left|\boldsymbol{\eta}_{m}(\cdot, \tau)\right|_{C^{2+\alpha}(\Omega)} \leqslant \Sigma_{m}(t)$, the last inequality implies

$$
\begin{aligned}
\Sigma_{n+1}(t) \leqslant & c_{1}\left(2 \int_{0}^{t} \Sigma_{n}(\tau) \mathrm{d} \tau+\int_{0}^{t}|\mathbf{w}(\cdot, \tau)|_{C^{\alpha+2}(\Omega)} \mathrm{d} \tau+\varepsilon\right) \Sigma_{n}(t) \\
& +c_{1} \int_{0}^{t} \Sigma_{n}(\tau) \mathrm{d} \tau+Y_{1}(t) .
\end{aligned}
$$

Assume that $T_{1}$ and $\varepsilon$ are so small that

$$
c_{1}\left(\int_{0}^{T_{1}}|\mathbf{w}(\cdot, \tau)|_{C^{2+\alpha}(\Omega)} \mathrm{d} \tau+\varepsilon\right) \leqslant \frac{1}{2} .
$$


Then

$$
\Sigma_{n+1}(t) \leqslant 4 c_{1} \Sigma_{n}(t) \int_{0}^{t} \Sigma_{n}(\tau) \mathrm{d} \tau+2 c_{1} \int_{0}^{t} \Sigma_{n+1}(\tau) \mathrm{d} \tau+c \varepsilon, \quad t<T_{1},
$$

and, by the Gronwall lemma,

$$
\begin{aligned}
\Sigma_{n+1}(t) & \leqslant e^{2 c_{1} T_{1}}\left(4 c_{1} \Sigma_{n}(t) \int_{0}^{t} \Sigma_{n}(\tau) \mathrm{d} \tau+c \varepsilon\right) \\
& \equiv 2 c_{2} \Sigma_{n}(t) \int_{0}^{t} \Sigma_{n}(\tau) \mathrm{d} \tau+c_{3} \varepsilon
\end{aligned}
$$

Integrating this estimate and setting

$$
R_{n}(t)=\int_{0}^{t} \Sigma_{n}(\tau) \mathrm{d} \tau
$$

we obtain

$$
R_{n+1}(t) \leqslant c_{2} R_{n}^{2}(t)+c_{3} \varepsilon T_{1}
$$

Now, let

$$
4 c_{2} c_{3} T_{1} \varepsilon<1
$$

and let

$$
x_{0}=\frac{1}{2 c_{2}}-\sqrt{\frac{1}{4 c_{2}^{2}}-\frac{c_{3} \varepsilon T_{1}}{c_{2}}}=\frac{2 c_{3} \varepsilon T_{1}}{1+\sqrt{1-4 c_{2} c_{3} T_{1} \varepsilon}}
$$

be a minimal root of the quadratic equation

$$
c_{2} X^{2}-X+c_{3} \varepsilon T_{1}=0 .
$$

It follows from (5.17) that if $R_{n}\left(T_{1}\right) \leqslant x_{0}$, then also $R_{n+1}\left(T_{1}\right) \leqslant x_{0}$. Hence, $R_{k}\left(T_{1}\right) \leqslant x_{0}$ for $k=1, \ldots, n+1$, further, in virtue of (5.16),

$$
\Sigma_{n+1}(t) \leqslant 2 c_{2} x_{0} \Sigma_{n}(t)+c_{3} \varepsilon=\frac{4 c_{2} c_{3} \varepsilon T_{1}}{1+\sqrt{1-4 c_{2} c_{3} T_{1} \varepsilon}} \Sigma_{n+1}+c_{3} \varepsilon,
$$

and

$$
\Sigma_{n+1}(t) \leqslant \frac{c_{3} \varepsilon}{\sqrt{1-4 c_{2} c_{3} T_{1} \varepsilon}} .
$$

This shows that under the hypotheses (5.13) and (5.18), we have

$$
\begin{aligned}
& \varepsilon \sup _{\tau<T_{1}}\left|\mathbf{u}_{n+1}(\cdot, \tau)\right|_{C^{\alpha}(\Omega)}+\sup _{\tau<T_{1}}\left|\mathbf{u}_{n+1}(\cdot, \tau)\right|_{C^{2+\alpha}(\Omega)}+\sup _{\tau<T_{1}}\left|q_{n+1}(\cdot, \tau)\right|_{C^{1+\alpha}(\Omega)} \\
& \quad \leqslant \varepsilon \sup _{\tau<T_{1}}\left|\mathbf{w}_{\tau}+\mathbf{V}_{\tau}\right|_{C^{\alpha}(\Omega)}+\sup _{\tau<T_{1}}|\mathbf{w}+\mathbf{V}|_{C^{2+\alpha}(\Omega)}+\sup _{\tau<T_{1}}|r+P|_{C^{1+\alpha}(\Omega)}+\frac{c_{3} \varepsilon}{\sqrt{1-4 c_{2} c_{3} T_{1} \varepsilon}},
\end{aligned}
$$

and, by virtue of (4.20) and (4.26),

$$
\int_{0}^{T_{1}}\left|\mathbf{u}_{n+1}(\cdot, \tau)\right|_{C^{2+\alpha}(\Omega)} \mathrm{d} \tau \leqslant+c \varepsilon+\int_{0}^{T_{1}}\left(|\mathbf{w}(\cdot, \tau)|_{C^{2+\alpha}(\Omega)}+|r|_{C^{1+\alpha}(\Omega)}\right) \mathrm{d} \tau,
$$


i.e. inequalities (5.13), $n=1,2, \ldots$, hold for small $\varepsilon$ and small $T_{1}$ independent of $\varepsilon$. Then (5.19) also holds for arbitrary $n$, and the successive approximations $\left(\boldsymbol{\eta}_{m}, \pi_{m}\right)$ are convergent to the solution $(\boldsymbol{\eta}, \pi)$ of the problem (1.19). It follows from (5.16) and from the above estimate for $R_{n}\left(T_{1}\right)$ that

$$
\varepsilon \sup _{\tau<T_{1}}\left|\boldsymbol{\eta}_{\tau}(\cdot, \tau)\right|_{C^{\alpha}(\Omega)}+\sup _{\tau<T_{1}}|\boldsymbol{\eta}(\cdot, \tau)|_{C^{2+\alpha}(\Omega)}+\sup _{\tau<T_{1}}|\pi(\cdot, \tau)|_{C^{1+\alpha}(\Omega)} \leqslant \frac{c_{3} \varepsilon}{\sqrt{1-4 c_{2} c_{3} \varepsilon}},
$$

which implies (1.20).

The solution which we have found is unique. The difference of two solutions $\psi=\boldsymbol{\eta}-\boldsymbol{\eta}^{\prime}$, $\rho=\pi-\pi^{\prime}$ satisfies the equations

$$
\begin{aligned}
& \varepsilon \boldsymbol{\psi}_{t}-\nabla_{w}^{2} \boldsymbol{\psi}+\nabla \rho=\mathcal{L}_{1}(\boldsymbol{\eta}, \pi)-\mathcal{L}_{1}\left(\boldsymbol{\eta}^{\prime}, \pi^{\prime}\right), \\
& \nabla_{w} \cdot \boldsymbol{\psi}=\mathcal{L}_{2}(\boldsymbol{\eta})-\mathcal{L}_{2}\left(\boldsymbol{\eta}^{\prime}\right), \\
& \left.\boldsymbol{\psi}\right|_{t=0}=0, \\
& \left.\Pi_{w} S(\boldsymbol{\psi}) \mathbf{n}_{w}\right|_{\Gamma}=\mathcal{L}_{3}(\boldsymbol{\eta})-\mathcal{L}_{3}(\boldsymbol{\eta}), \\
& \mathbf{n}_{w} \cdot T_{w}(\boldsymbol{\psi}, \rho) \mathbf{n}_{w}-\left.\sigma \mathbf{n}_{w} \cdot \Delta_{w}(t) \int_{0}^{t} \boldsymbol{\psi} \mathrm{d} \tau\right|_{\Gamma}=\mathcal{L}(\boldsymbol{\eta}, \pi)-\mathcal{L}\left(\boldsymbol{\eta}^{\prime}, \pi^{\prime}\right) .
\end{aligned}
$$

Repeating the above arguments, we obtain for the norm

$$
Y(t)=\varepsilon \sup _{\tau<t}|\psi(\cdot, \tau)|_{C^{\alpha}(\Omega)}+\sup _{\tau<t}|\psi(\cdot, \tau)|_{C^{2+\alpha}(\Omega)}+\sup _{\tau<t}|\rho(\cdot, \tau)|_{C^{1+\alpha}(\Omega)}
$$

the estimate similar to (5.14), i.e.

$Y(t) \leqslant c_{1}\left[\int_{0}^{t}\left(|\boldsymbol{\eta}(\cdot, \tau)|_{C^{2+\alpha}(\Omega)}+\left|\boldsymbol{\eta}^{\prime}(\cdot, \tau)\right|_{C^{2+\alpha}(\Omega)}+|\mathbf{w}(\cdot, \tau)|_{C^{2+\alpha}(\Omega)}\right) \mathrm{d} \tau+\varepsilon\right] Y(t)+c_{1} \int_{0}^{t} Y(\tau) \mathrm{d} \tau$.

Let us assume that $\left(\boldsymbol{\eta}^{\prime}, q^{\prime}\right)$ is the solution constructed above. Then (5.20) implies

$$
Y(t) \leqslant 2 c_{1} Y(t)\left(\int_{0}^{t} Y(\tau) \mathrm{d} \tau+c \varepsilon\right)+2 c_{1} \int_{0}^{t} Y(\tau) \mathrm{d} \tau,
$$

from which it follows that $Y(t)=0$, if $\varepsilon$ is sufficiently small. The theorem is proved.

\subsection{Extension of the solution of the problem (1.6) to the interval $t \in\left(0, T_{0}\right)$}

We extend $(\mathbf{u}, q)$ outside the interval $\left(0, T_{1}\right)$ by introducing new Lagrangean coordinates, as it has been already done above. Let $\xi^{\prime}=X_{w}\left(\xi, T_{1}\right)$. In these coordinates, equations (1.8) can be written as follows:

$$
\begin{gathered}
-\nabla_{w}^{\prime 2} \mathbf{w}^{\prime}+\nabla_{w}^{\prime} r^{\prime}+\ell_{w}^{\prime}\left(\mathbf{w}^{\prime}\right)=\sum_{k=1}^{6} \mu_{k} \varphi\left(X_{w}^{\prime}\left(\xi^{\prime}, t\right)\right), \\
\nabla_{w}^{\prime} \cdot \mathbf{w}^{\prime}=0, \quad \xi^{\prime} \in \Omega^{\prime} \equiv X_{w}\left(\Omega, T_{1}\right), t>T_{1}, \\
T_{w}^{\prime}\left(\mathbf{w}^{\prime}, q^{\prime}\right) \mathbf{n}_{w}^{\prime}-\left.\Delta_{w}^{\prime}(t) \mathbf{X}_{w}^{\prime}\left(\xi^{\prime}, t\right)\right|_{\Gamma^{\prime}}=0 .
\end{gathered}
$$


Here $\Gamma^{\prime}=\partial \Omega^{\prime}, \mathbf{w}^{\prime}\left(\xi^{\prime}, t\right)=\mathbf{w}\left(X_{w}^{-1}\left(\xi^{\prime}, T_{1}\right), t\right), r^{\prime}\left(\xi^{\prime}, t\right)=r\left(X_{w}^{-1}\left(\xi^{\prime}, T_{1}\right), t\right), \mathbf{X}_{w}^{\prime}\left(\xi^{\prime}, t\right)=$ $\xi^{\prime}+\int_{T_{1}}^{t} \mathbf{w}^{\prime}\left(\xi^{\prime}, \tau\right) \mathrm{d} \tau\left(t>T_{1}\right), \nabla_{w}^{\prime}=\mathcal{A}^{(w)^{\prime}} \nabla_{\xi^{\prime}}, \mathcal{A}^{(w)^{\prime}}$ is the matrix of cofactors of $a_{i j}^{\prime}=\delta_{i j}+$ $\int_{T_{1}}^{t} \frac{\partial w_{i}^{\prime}}{\partial \xi_{j}^{\prime}} \mathrm{d} \tau, T_{w}^{\prime}=-p I+S_{w}^{\prime}, S_{w}^{\prime}\left(\mathbf{w}^{\prime}\right)=\nabla_{w}^{\prime} \mathbf{w}+\left(\nabla_{w}^{\prime} \mathbf{w}^{\prime}\right)^{T}, \mathbf{n}_{w}^{\prime}$ and $\Delta_{w}^{\prime}$ are the normal vector and the Laplace-Beltrami operator on $\Gamma_{w}^{\prime}(t)=X_{w}^{\prime}\left(\Gamma^{\prime}\right)$, finally,

$$
\ell_{w}^{\prime}\left(\mathbf{w}^{\prime}\right)=\sum_{k=1}^{6} \int_{\Omega^{\prime}} \mathbf{w}^{\prime}\left(\xi^{\prime \prime}, t\right) \cdot \boldsymbol{\varphi}_{k}\left(X_{w}^{\prime}\left(\xi^{\prime \prime}, t\right)\right) \mathrm{d} \xi^{\prime \prime} \boldsymbol{\varphi}_{k}\left(X_{w}^{\prime}\left(\xi^{\prime}, t\right)\right)
$$

Equations (1.6) can be written in a similar way, namely,

$$
\begin{gathered}
\varepsilon \mathbf{u}_{t}^{\prime}+\ell_{u}^{\prime}\left(\mathbf{u}^{\prime}\right)-\nabla_{u}^{\prime 2} \mathbf{u}^{\prime}+\nabla_{u}^{\prime} q^{\prime}=\sum_{k=1}^{6} \mu_{k} \boldsymbol{\varphi}_{k}\left(X_{u}^{\prime}\left(\xi^{\prime}, t\right)\right), \\
\nabla_{u}^{\prime} \cdot \mathbf{u}^{\prime}=0, \quad\left(\xi^{\prime} \in \Omega^{\prime}, \quad t>T_{1}\right), \\
\left.\mathbf{u}^{\prime}\left(\xi^{\prime}, t\right)\right|_{t=T_{1}}=\mathbf{u}\left(X_{w}^{-1}\left(\xi^{\prime}, T_{1}\right), T_{1}\right), \\
T_{u}^{\prime}\left(\mathbf{u}^{\prime}, q^{\prime}\right) \mathbf{n}^{\prime}-\left.\Delta_{u}^{\prime}(t) \mathbf{X}_{u}^{\prime}\right|_{\Gamma}=0
\end{gathered}
$$

where $\mathbf{u}^{\prime}\left(\xi^{\prime}, t\right)=\mathbf{u}\left(X_{w}^{-1}\left(\xi^{\prime}, T_{1}\right), t\right), q^{\prime}\left(\xi^{\prime}, t\right)=q\left(\left(X_{w}^{-1}\left(\xi^{\prime}, T_{1}\right), t\right)\right.$,

$$
\ell_{w}^{\prime}\left(\mathbf{u}^{\prime}\right)=\sum_{k=1}^{6} \int_{\Omega^{\prime}} \mathbf{u}^{\prime}\left(\xi^{\prime \prime}, t\right) \cdot \boldsymbol{\varphi}_{k}\left(X_{u}^{\prime}\left(\xi^{\prime \prime}, t\right) \mathrm{d} \xi^{\prime \prime} \boldsymbol{\varphi}_{k}\left(X_{u}^{\prime}\left(\xi^{\prime}, t\right)\right)\right.
$$

and $\mathbf{X}_{u}^{\prime}, \nabla_{u}^{\prime}$ are computed according to the formulas

$$
\begin{gathered}
\mathbf{X}_{u}^{\prime}\left(\xi^{\prime}, t\right)=\left.\mathbf{X}_{u}(\xi, t)\right|_{\xi=X_{w}^{-1}\left(\xi^{\prime}, T_{1}\right)}=\xi^{\prime}+\int_{T_{1}}^{t} \mathbf{u}^{\prime}\left(\xi^{\prime}, \tau\right) \mathrm{d} \tau+\mathbf{R}\left(\xi^{\prime}\right), \\
\mathbf{R}\left(\xi^{\prime}\right)=\left.\int_{0}^{T_{1}}(\mathbf{u}(\xi, \tau)-\mathbf{w}(\xi, \tau)) \mathrm{d} \tau\right|_{\xi=X_{w}^{-1}\left(\xi^{\prime}, T_{1}\right)}, \\
\nabla_{u}^{\prime}=\left.\nabla_{u}\right|_{\xi=X_{w}^{-1}\left(\xi^{\prime}, T_{1}\right)}=\left.\mathcal{A}^{(u)}(\xi, t) a^{(w) T}\left(\xi, T_{1}\right)\right|_{\xi=X_{w}^{-1}\left(\xi^{\prime}, T_{1}\right)} \nabla_{\xi^{\prime}}
\end{gathered}
$$

where

$$
a^{(w)}=\left(\delta_{i j}+\int_{0}^{T_{1}} \frac{\partial w_{i}(\xi, \tau)}{\partial \xi_{j}} \mathrm{~d} \tau\right)_{i, j=1,2,3} .
$$

It follows from (5.21) and (5.22) that

$$
\begin{gathered}
\mathbf{X}_{u}^{\prime}\left(\xi^{\prime}, t\right)-\mathbf{X}_{w}^{\prime}\left(\xi^{\prime}, t\right)=\int_{T_{1}}^{t}\left[\mathbf{u}^{\prime}\left(\xi^{\prime}, \tau\right)-\mathbf{w}^{\prime}\left(\xi^{\prime}, \tau\right)\right] \mathrm{d} \tau+\mathbf{R}\left(\xi^{\prime}\right), \\
\nabla_{u}^{\prime}-\nabla_{w}^{\prime}=\left(\left.\left(\mathcal{A}^{(u)}(\xi, t)-\mathcal{A}^{(w)}(\xi, t)\right) a^{(w) T}\left(\xi, T_{1}\right)\right|_{\xi=X_{w}^{-1}\left(\xi^{\prime}, T_{1}\right)}\right) \nabla_{\xi^{\prime}} .
\end{gathered}
$$

By virtue of (1.15) and (5.20),

$$
|\mathbf{R}|_{C^{2+\alpha}\left(\Omega^{\prime}\right)} \leqslant c \int_{0}^{T_{1}}|\mathbf{u}-\mathbf{w}|_{C^{2+\alpha}\left(\Omega^{\prime}\right)} \mathrm{d} \tau \leqslant c \varepsilon,
$$


hence,

$$
\left|X_{u}^{\prime}(\cdot, t)-X_{w}^{\prime}(\cdot, t)\right|_{C^{2+\alpha}\left(\Omega^{\prime}\right)} \leqslant c\left(\varepsilon+\int_{T_{1}}^{t}\left|\mathbf{u}^{\prime}-\mathbf{w}^{\prime}\right|_{C^{2+\alpha}\left(\Omega^{\prime}\right)} \mathrm{d} \tau\right)
$$

and similar estimate holds for the coefficients of the operator (5.23). Estimates (4.6), (4.7), and (4.9) in new coordinates are conserved but $\int_{0}^{t}|\mathbf{u}-\mathbf{w}|_{C^{k+\alpha}(\Omega)} \mathrm{d} \tau$ should be replaced everywhere with $\int_{T_{1}}^{t}\left|\mathbf{u}^{\prime}-\mathbf{w}^{\prime}\right|_{C^{k+\alpha}\left(\Omega^{\prime}\right)} \mathrm{d} \tau+\varepsilon$.

Let $\mathbf{V}^{\prime}, P^{\prime}$ be a solution of a linear problem

$$
\begin{gathered}
\varepsilon \mathbf{V}_{t}^{\prime}-\nabla_{w}^{\prime 2} \mathbf{V}^{\prime}+\ell_{w}^{\prime}\left(\mathbf{V}^{\prime}\right)+\nabla_{w} P^{\prime}=0, \\
\nabla_{w}^{\prime} \cdot \mathbf{V}^{\prime}=0, \quad \xi \in \Omega^{\prime}, \quad t \in\left(T_{1}, T_{0}\right), \\
\left.\mathbf{V}^{\prime}\right|_{t=T_{1}}=\mathbf{u}^{\prime}\left(\xi^{\prime}, T_{1}\right)-\mathbf{w}^{\prime}\left(\xi^{\prime}, T_{1}\right), \\
\left.T_{w}^{\prime}\left(\mathbf{V}^{\prime}, P^{\prime}\right) \mathbf{n}_{w}^{\prime}\right|_{\Gamma}=0 .
\end{gathered}
$$

For the differences

$$
\eta^{\prime}=\mathbf{u}^{\prime}-\mathbf{w}^{\prime}-\mathbf{V}^{\prime}, \quad \pi^{\prime}=q^{\prime}-r^{\prime}-P^{\prime}
$$

we obtain the problem similar to (1.19), namely,

$$
\begin{gathered}
\varepsilon \boldsymbol{\eta}_{t}^{\prime}-\nabla_{w}^{\prime 2} \boldsymbol{\eta}^{\prime}+\ell_{w}^{\prime}\left(\boldsymbol{\eta}^{\prime}\right)+\nabla_{w}^{\prime} \pi^{\prime}=-\varepsilon \mathbf{w}_{t}^{\prime}+\mathcal{L}^{\prime}{ }_{1}\left(\boldsymbol{\eta}^{\prime}, \pi^{\prime}\right), \\
\nabla_{w}^{\prime} \cdot \boldsymbol{\eta}^{\prime}=\mathcal{L}_{2}^{\prime}{ }_{2}\left(\boldsymbol{\eta}^{\prime}\right), \quad \xi \Omega^{\prime}, \quad t \in\left(T_{1}, T_{0}\right), \\
\left.\boldsymbol{\eta}^{\prime}\right|_{t=T_{1}}=0, \\
\left.\Pi_{w}^{\prime} S_{w}^{\prime}\left(\boldsymbol{\eta}^{\prime}\right) \mathbf{n}_{w}^{\prime}\right|_{\Gamma} ^{\prime}=\mathcal{L}^{\prime}{ }_{3}\left(\boldsymbol{\eta}^{\prime}\right), \\
\mathbf{n}_{w}^{\prime} \cdot T_{w}^{\prime}\left(\boldsymbol{\eta}^{\prime}, \pi^{\prime}\right) \mathbf{n}_{w}^{\prime}-\left.\mathbf{n}_{w}^{\prime} \cdot \Delta_{w}^{\prime}(t)\left(\mathbf{X}_{u}^{\prime}-\mathbf{X}_{w}^{\prime}\right)\right|_{\Gamma} ^{\prime}=\mathcal{L}^{\prime}\left(\boldsymbol{\eta}^{\prime}, \pi^{\prime}\right)
\end{gathered}
$$

where

$$
\begin{gathered}
\mathcal{L}^{\prime}{ }_{1}\left(\boldsymbol{\eta}^{\prime}, \pi^{\prime}\right)=\left(\nabla_{u}^{\prime 2}-\nabla_{w}^{\prime 2}\right) \mathbf{u}^{\prime}-\left(\nabla_{u}^{\prime}-\nabla_{w}^{\prime}\right) q^{\prime} \\
+\ell_{w}^{\prime}\left(\mathbf{u}^{\prime}\right)-\ell_{u}^{\prime}\left(\mathbf{u}^{\prime}\right)+\sum_{k=1}^{6} \mu_{k}\left(\varphi_{k}\left(X_{u}^{\prime}(\xi, t)\right)-\boldsymbol{\varphi}_{k}\left(X_{w}^{\prime}(\xi, t)\right),\right. \\
\mathcal{L}^{\prime}{ }_{2}\left(\boldsymbol{\eta}^{\prime}\right)=\left(\nabla_{w}^{\prime}-\nabla_{u}^{\prime}\right) \cdot \mathbf{u}^{\prime}, \\
\mathcal{L}_{3}^{\prime}\left(\eta^{\prime}\right)=\Pi_{w}^{\prime}\left(\Pi_{w}^{\prime} S_{w}^{\prime}\left(\mathbf{u}^{\prime}\right) \mathbf{n}_{w}^{\prime}-\Pi_{u}^{\prime} S_{u}^{\prime}\left(\mathbf{u}^{\prime}\right) \mathbf{n}_{u}^{\prime}\right), \\
\mathcal{L}^{\prime}\left(\boldsymbol{\eta}^{\prime}, \pi^{\prime}\right)=\mathbf{n}_{w}^{\prime} \cdot\left(T_{w}^{\prime}\left(\mathbf{u}^{\prime}, q^{\prime}\right) \mathbf{n}_{w}^{\prime}-T_{u}^{\prime}\left(\mathbf{u}^{\prime}, q^{\prime}\right) \mathbf{n}_{u}^{\prime}\right)+\mathbf{n}_{w}^{\prime} \cdot\left(\Delta_{u}^{\prime}(t)-\Delta_{w}^{\prime}(t)\right) \mathbf{X}_{u}^{\prime}(\xi, t)
\end{gathered}
$$

and $\mathbf{u}^{\prime}=\mathbf{w}^{\prime}+\mathbf{V}^{\prime}+\eta^{\prime}, \quad q^{\prime}=r^{\prime}+P^{\prime}+\pi^{\prime}$. The presence of the extra term $c \varepsilon$ in (4.6)-(4.9) cannot be an obstacle for the construction of the solution of problem (5.24) in the interval $t \in\left(T_{1}, 2 T_{1}\right)$, as above, by a successive approximation procedure, since, by virtue of (1.14), (4.20), and (5.20), we have now a uniform estimate for $\left(\mathbf{V}^{\prime}, P^{\prime}\right)$ :

$$
\begin{gathered}
\varepsilon \sup _{T_{1}<\tau<t}\left|\mathbf{V}_{\tau}^{\prime}(\cdot, \tau)\right|_{C^{\alpha}\left(\Omega^{\prime}\right)}+\sup _{T_{1}<\tau<t}\left|\mathbf{V}^{\prime}(\cdot, \tau)\right|_{C^{2+\alpha}\left(\Omega^{\prime}\right)}+\sup _{T_{1}<\tau<t}\left|P^{\prime}(\cdot, \tau)\right|_{C^{l+1}(\Omega)} \\
\leqslant c\left|\mathbf{u}^{\prime}\left(\cdot, T_{1}\right)-\mathbf{w}^{\prime}\left(\cdot, T_{1}\right)\right|_{C^{2+\alpha}\left(\Omega^{\prime}\right)} \leqslant c \varepsilon .
\end{gathered}
$$

Repeating this procedure several times, we can extend $(\mathbf{u}, q)$ onto the whole interval $\left(0, T_{0}\right)$. 


\section{Acknowledgments}

This paper was written during the stay of the author at Centro de Matematica e Aplicaçoes Fundamentais of the University of Lisbon whose hospitality is gratefully acknowledged. The author is happy to thank Professor V. V. Pukhnachov for fruitful detailed discussions of the subject.

\section{REFERENCES}

1. Bizhanova, G. I. \& Solonnikov, V. A. Some model problems for parabolic equations with time derivative in boundary conditions. Algebra Anal. 6, (1994) 30-50.

2. Golovkin, K. K. Certain conditions of smoothness of a function of several variables and estimates of convolution operators. Doklady Akad. Nauk. SSSR 139, (1961) 524-527.

3. Golovin, K. K. \& Solonnikov, V. A. Estimates of integral operators in translation-invariant norm. Trudy Mat. Inst. Steklov 70, (1964) 45-58.

4. GÜNThER, M. \& Prokert, G. Existence results for the quasistationary motion of a free capillary liquid drop. Zeitschr. für Anal. und ihre Anwend. 16, (1997) 311-348.

5. LAVRENT'EVA, O. M. The motion of a rotating ring of a viscous capillary fluid, Manuscript No. 7562-84, deposed at VINITI, 1984 (Russian).

6. LUNARDI, A. Maximal space regularity in nonhomogeneous initial boundary value parabolic problem. Numer. Funct. Anal. Optimiz. 10, (1989) 323-349.

7. Moghilevskit, I. Sh. \& Solonnikov, V. A. Solvability of a non-coercive initial-boundary value problem for the nonstationary Stokes system in Hölder spaces (the case of half-space). Zeitschr. für Anal. und ihre Anwend. 8, (1989) 327-349.

8. MoghilevskiI, I. SH. \& Solonnikov, V. A. On the solvability of an evolution free boundary problem for the Navier-Stokes equations in the Hölder spaces of functions, Mathematical problems related to the Navier-Stokes equations, Series on advances in math. for appl. sciences 11, (1992) 105-181.

9. Puknnachov, V. V. Quasistationary approximation in the problem of motion of an isolated volume of a viscous incompressible capillary liquid. Prikl. Mat. Makh. (1999) (submitted).

10. Solonnikov, V. A. Estimates of solutions of initial-boundary value problem for a linear nonstationary system of the Navier-Stokes equations. Zap. nauchn. semin. LOMI 59, (1976) 178-254.

11. Solonnikov, V. A. On the solvability of the second initial-boundary value problem for the linear timedependent system of the Navier-Stokes equations. Zap. nauchn. semin. LOMI 69, (1977) 200-218.

12. Solonnikov, V. A. On nonstationary motion of a finite liquid mass bounded by a free surface. Zap. nauchn. semin. LOMI 152, (1986) 137-157.

13. Solonnikov, V. A. On the transient motion of an isolated volume of viscous incompressible fluid. Izv. Akad. Nauk SSSR, ser. mat. 51, (1987) 1065-1087.

14. Solonnikov, V. A. On the evolution of an isolated volume of viscous incompressible capillary fluid for large values of time. Vestnik Leningr. Univ. ser 1 3, (1987) 49-55.

15. Solonnikov, V. A. On nonstationary motion of a finite isolated mass of self-gravitating fluid. Algebra Anal. 1(1), (1989) 207-249.

16. Solonnikov, V. A. On initial-boundary value problem for the Stokes system arising in the study of a free boundary problem. Trudy Mat. Inst. Steklov 188, (1990) 150-188.

17. Solonnikov, V. A. On the quasistationary approximation in the problem of motion of a capillary drop. In Escher, J. \& SimonetT, G (eds), Topics in nonlinear analysis-The Herbert Amann anniversary volume, pp. 643-671. Birkhauser (1999).

18. Solonnikov, V. A. Estimates of solution of the second initial-boundary value problem for the Stokes system in the space of functions with Hölder continuous derivatives with respect to spatial variables. Zap. nauchn. semin. POMI (1999) (submitted). 